\title{
MÖSSBAUER STUDIES OF THE TRANSITION ELEMENT HALIDES
}

\author{
JOHN FRANCIS CAYANAGH*
}

Physics Department

Purdue University

Lof ayotte, Indiona

\footnotetext{
- This report is bosed on a thesis submitted in partial fulfillment of the requirements of the degree of Doctor of Philosophy of Purdue University.
} 


\section{DISCLAIMER}

This report was prepared as an account of work sponsored by an agency of the United States Government. Neither the United States Government nor any agency Thereof, nor any of their employees, makes any warranty, express or implied, or assumes any legal liability or responsibility for the accuracy, completeness, or usefulness of any information, apparatus, product, or process disclosed, or represents that its use would not infringe privately owned rights. Reference herein to any specific commercial product, process, or service by trade name, trademark, manufacturer, or otherwise does not necessarily constitute or imply its endorsement, recommendation, or favoring by the United States Government or any agency thereof. The views and opinions of authors expressed herein do not necessarily state or reflect those of the United States Government or any agency thereof. 


\section{DISCLAIMER}

Portions of this document may be illegible in electronic image products. Images are produced from the best available original document. 


\author{
United States Atomic Energy Commission \\ Technical Report No. Co0-AT(11-1)-1616-11
}

Mo̊ssbauer Studies of the Transition Element Halides

John Francis Cavanagh*

Physics Department, Purdue University

Lafayette, Indiana

\begin{abstract}
*This report is based on a thesis submitted in partial fulfillment of the requirements of the degree of Doctor of Philosophy at Purdue University.
\end{abstract}

\title{
LEGAL NOTICE
}

Thls report was prepared as an account of Government sponsored work. Nelther the Unlted States, nor the Commisslon, nor any person acting on betalt of the Commisslon:

$A$. Makos any war ranty or representation. expressed or implied, wh rapeet to the aceuracy, completeness, orparatus, method, or process diaclosed in this report may not intringe

privately owned rights; or privately owned rights; or
B. Aseumes any liabitites with respect to the use of, or lor dnmages rest.

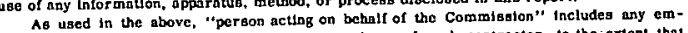
ployee or contractor of the commission, or employee of such contractor. wo the extent that such employee or contractor of the commlasion, or employe of ats employment or contract with the Commigsion, or tis employment with sucb contractor. 


\section{ACKNOWLEDGMENTS}

I would like to express my appreciation to Professor James G. Mullen for the invaluable suggestions and advice he offered during the course of this research. The assistance of my fellow graduate students, especially Dr. Hang Nam $\mathrm{OK}$ and $\mathrm{Mr}$. Robert C. Knauer, is also gratefully acknowledged. This work was supported in part by the United States Atomic Energy Commission. 
TABLE OF CONTENTS

Page

LIST OF TABLES. . . . . . . . . . . . . . . . . . v

LIST OF FIGURES . . . . . . . . . . . . . . . . . . vi

LIST OF SYMBOLS . . . . . . . . . . . . . . . . . . . ix

ABSTRACT. . . . . . . . . . . . . ...... xi

I. INTRODUCTION. . . . . . . . . . . . . . . . I

II. THEORY . . . . . . . . . . . . . . . . 10

A. Recoil-free Fraction ................ 10

B. Crystal Perturbations of Nuclear Levels. . . . . . . . 12

C. Karyagin-Goldanski Effect. ............. 22

D. Crystal Field Theory ............. 26

III. EXPER IMENTAL TECHNIQUES . . . . . . . . . . . . 33

A. Sample Preparation . . . . . . . . . . . . 33

B. Mössbauer Spectrometers. . . . . . . . . . 36

C. Temperature Measurement and Control. . . . . . . . 40

IV . EXPERIMENTAL RESULTS . . . . . . . . . . . . . 48

A. Data Reduction and Experimental Errors . . . . . . . 48

B. Mössbauer Spectra Above the Néel Temperature . . . . . 50

1. $\mathrm{CoCl}_{2}$. . . . . . . . . . . . . . 50

2. $\mathrm{CoF}_{2} \cdot$. . . . . . . . . . . . . . . . 54

C. Mössbauer Spectra Below the Néel Temperature . . . . . 59

1. $\mathrm{CoC}_{2}$. . . . . . . . . . . . . . . . 59

2. $\mathrm{CoF}_{2} \cdot . \cdot . \cdot . \cdot . \cdot . \cdot . \cdot . . . . .63$

V. DISCUSSION OF EXPERIMENTAL RESULTS. . . . . . . . . . 69

A. $\operatorname{CoCl}_{2}$ Spectra Above the Néel Temperature . . . . . . 69

B. Karyagin-Goldanski. Effect in $\mathrm{CoCl}_{2}$. . . . . . . . . 71

C. $\mathrm{CoF}_{2}$ Spectra Above the Nêel Temperature. . . . . . . 73

D. Hyperfine Field Parameters ... . . . . . . . 82

VI. SUMMARY. . . . . . . . . . . . . . . . 91 
TABLE OF CONTENTS (Contd)

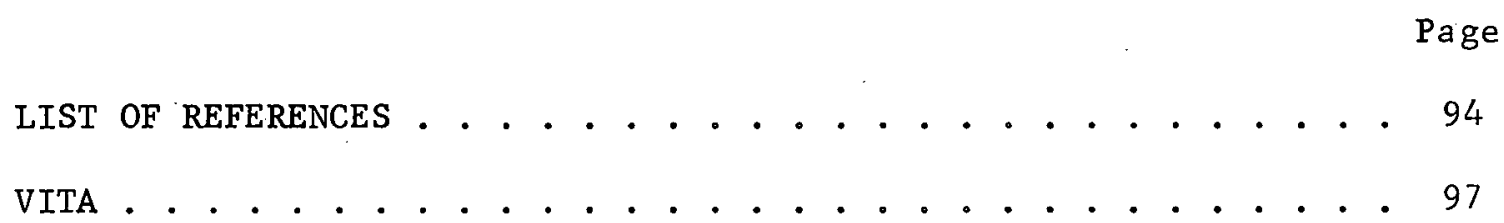


1. General form of the solution for the first excited state of $\mathrm{Fe}^{57}$ under a combined magnetic dipole and electric quadrupole interaction and the positions and intensities of the relative Mössbauer lines . . . . . . 18

2. Experimentally measured parameters of the Mössbauer spectra of $\mathrm{CoCl}_{2}$ above the Néel temperature . . . . . . 52

3. Experimentally measured parameters of the Mössbauer spectra of $\mathrm{CoF}_{2}$ above the Néel temperature. . . . . . . 56

4. Eigenenergies and eigenstates of the $\dot{\mathrm{F}} \mathrm{C}^{2+} \mathrm{d}$ level due to a trigonal field and spin-orbit interaction

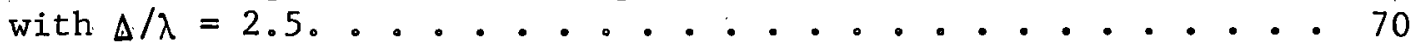




\section{L.IST OF FIGURES}

\section{Figure}

Page

1. Decay scheme of $\mathrm{Co}^{57}$. . . . . . . . . . . . . . 3

2. Nuclear level splitting and resultant Mössbauer spectra for $\mathrm{Fe}^{57}$. a) electric quadrupole interaction, b) magnetic dipole interaction, . c) combined electric quadrupole and magnetic dipole interaction . . . . . . . . . . . . . . . . . 5

3. Calculated quadrupole doublet intensity ratio for an axially symetric electric field gradient as a function of $e^{2}=n^{2}\left[\left\langle z^{2}\right\rangle-\left\langle x^{2}\right\rangle\right] \ldots \ldots$

4. Quadrupole splitting reduction factor, $F(\Delta, \lambda, T)$, as a function of $\mathrm{kT} / \lambda$ with $\Delta / \lambda$ as a parameter for the case of an axially symmetric crystal field with

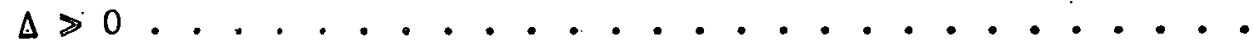

5. Schematic diagram of the apparatus used in the

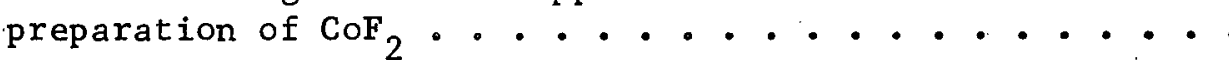

6. Schematic diagram of the rapid scan Mössbauer crank spectrometer. . . . . . . . . . . . . . . . .

7. Schematic diagram of the constant velocity

Mössbauer spectrometer. . . . . . . . . . . . . .

8. Radioactive sample holder and helium dewar cold finger mounting . . . . . . . . . . . . . . . . . .

9. Block diagram of the electronic temperature controller and schematic diagram of a typical amplifier stage . . . . . . . . . . . . . . . . . . .

10. Detailed schematic diagram of the temperature controller phase sensitive detector . . . . . . . . . .

11. Mössbauer spectra of $\mathrm{CoCl}_{2}$. as a function of temperature above the Néel temperature. The same vertical scale is used for the data on the left and the larger scale applies to the data between $373^{\circ} \mathrm{K}$ and $670^{\circ} \mathrm{K} . . . . . . . . . . . . . . .$. 
12. Temperature dependence of the quadrupole splitting of $\mathrm{Fe}^{57}$ in $\mathrm{CoCl}_{2}$. The solid line was calculated with the use of erystal fleld theory. . . . ... . . . . 53

13. Mössbauer spectra of CoF, as a function of temperature above the Nefed temperature. The game vertical seale 1s used throughout. . . . . . . . . . . . . . . 55

14. X-ray powder diffration pattern of Cof. The sample was prepared at $700^{\circ} \mathrm{C}$, and Mo K radfation wal used in determining the powder patteqn . . . . . . . . . 58

15. Mössbauer apectra of Co: dopad wh both $\mathrm{Co}^{57}$ and $\mathrm{Fe}^{57}$. The data (a) and (c) resulted from using the sample as a source, and the data (b) and (d) rasultad rom using the sample as an absorber . . . . . . . . . . . . 60

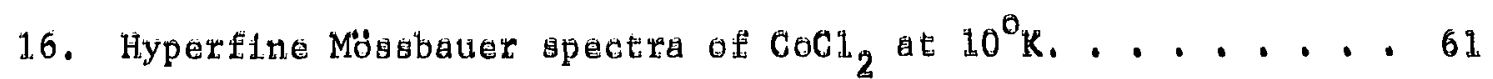

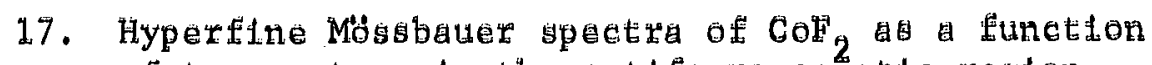
of temperature fin the antferromagntate region. . . . . . 64

18. Néél temperature determitution tor Cor by measuring

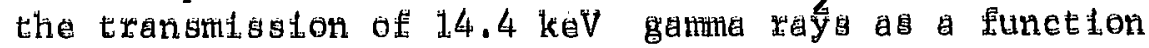
of temperature at zero relativa valoetty. . . . . . . . :66

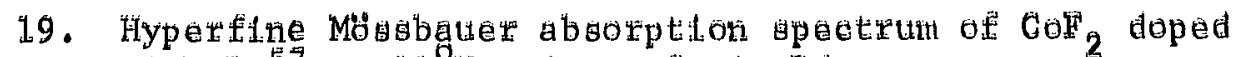

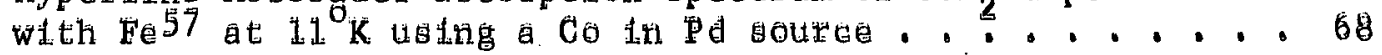

20. Goldandki. Karyagin a intengity ratio as a function of enperatura: b) asymatry parameter as a function ot tempature; sold

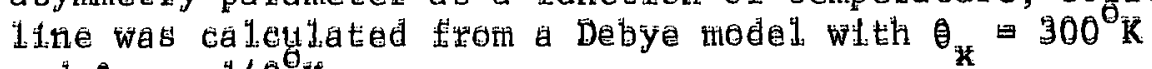

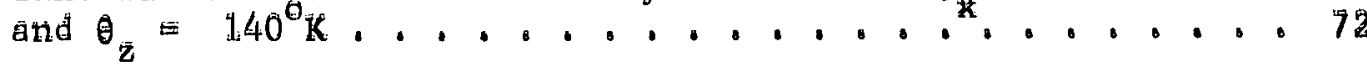

21. Tetrparature dependence of the retative area undex the speetral. paka th Cor a a a function of temparature.

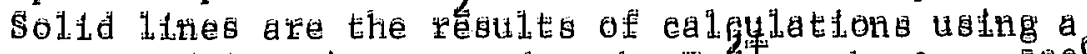
Debye model. a) areg under the te gak,

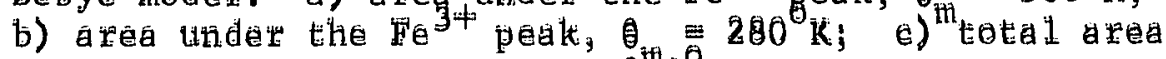




22. Ratio of the area under the $\mathrm{Fe}^{3+}$ and $\mathrm{Fe}^{2+}$ peaks as a function of temperature. The solid line is the ratio of recoil-free fractions calculated from a

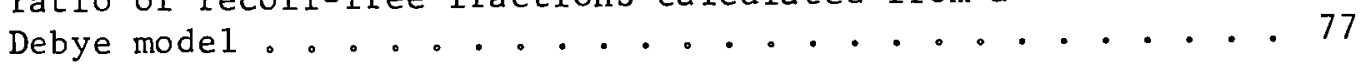

23. First moment of the $\mathrm{CoF}_{2}: \mathrm{Co}^{57}$ Mo̊ssbauex spectra as a function of temperature. . . . . . . . . . . 78

24. Experimental Móssbauer hyperfine spectra and the resonant emission spectra calculated from estimates of the hyperfine field parameters. The position and intensities of the spectral lines are indicated schematically。 a) $\mathrm{CoF}_{2}, \mathrm{~T}=11^{\circ} \mathrm{K}$. b) $\mathrm{CoCl}_{2}$, $\mathrm{T}=10^{\circ} \mathrm{K}$. 


\section{LIST OF SYMBOLS}

Symbo1

$|n\rangle$

$x$

$\mathbf{u}$

$\mathbf{R}$

$\theta_{\mathrm{m}}$

$\mathrm{S}$

$\delta$

Q

$\mathcal{K}$

$\mathrm{v}_{\mathrm{xx}}, \mathrm{v}_{\mathrm{yy}}, \mathrm{v}_{\mathrm{zz}}$

eq

$\eta$

$g_{0}, g_{1}$

- $\mu_{\mathrm{N}}$

$\mathrm{H}$

$\theta, \phi$
Name

Recoil-free fraction

Wave function describing a crystal lattice

Gamma ray wave number

Displacement of an atom from its equilibrium position in a lattice

Recoil energy associated with the emission or absorption of a gamma ray by a nucleus

Characteristic Mössbauer Debye temperature

Area under a resonant absorption spectral line

Isomer shift of a Mössbauer spectra

Nuclear qùadrupole moment tensor

Magnetic dipole and electric quadrupole Hamiltonian

Principal axes values of an electric field gradient tensor at the nucleus

Maximum of the three principal electric field gradient tensor components

Asymmetry parameter describing the electric field gradient tensor.

Nuclear g-factors for the ground and first excited states of $\mathrm{Fe}^{57}$

Nuclear magneton

Magnetic field at a nucleus

Polar and azimuthal angles describing the relative orientation of the magnetic field and the principal axes of the electric field gradient at a nucleus 


\begin{tabular}{|c|c|}
\hline Symbo1 & Name \\
\hline $\mathrm{P}$ & $\mathrm{e}^{2} \mathrm{qQ} / 6 \mathrm{~g}_{1} \mu_{\mathrm{N}} \mathrm{H}$ \\
\hline$E_{i}$ & Eigenenergy of the ith nuclear state \\
\hline$a_{i j}$ & $\begin{array}{l}\text { Matrix element of the unitary transformation } \\
\text { which diagonalizes } \mathfrak{F}\end{array}$ \\
\hline$\epsilon^{2}$ & $\begin{array}{l}\text { Parameter which describes the anisotropy in the } \\
\text { crystal binding }\end{array}$ \\
\hline$I_{\pi}, I_{\sigma}$ & $\begin{array}{l}\text { The intensity of the gamma rays arising from an } \\
\mathrm{m}= \pm 3 / 2 \text { to } \mathrm{m}= \pm 1 / 2 \text { and an } \mathrm{m}= \pm 1 / 2 \text { to } \\
\mathrm{m}= \pm 1 / 2 \text { nuclear transition }\end{array}$ \\
\hline $\mathrm{V}$ & Crystal field potential \\
\hline$\psi_{\mathrm{m}}$ & $\begin{array}{l}\text { Orbital wavefunctions of the free }{ }^{5} \mathrm{D} \text { ferrous } \\
\text { ion state }\end{array}$ \\
\hline$\lambda$ & Spin-orbit coupling constant \\
\hline$\Delta$ & Trigonal crystal field splitting factor \\
\hline$\varphi_{\mathrm{m}}$ & Orbital. wavefunctions for the ferrous ion $\mathrm{d}_{\epsilon}$ state \\
\hline$x_{j}$ & Spin wavefunctions for $\operatorname{spin} \overline{\mathrm{S}}=2$ \\
\hline $\bar{l}$ & Pseudo angular momentum \\
\hline$\alpha^{2}$ & Covalency reduction factor \\
\hline $\mathrm{F}(\Delta, \lambda, \mathrm{T})$ & Statistical quadrupole reduction factor \\
\hline$I(v)$ & $\begin{array}{l}\text { Measured gamma ray transmission as a function } \\
\text { of velocity }\end{array}$ \\
\hline$\Gamma$ & Mo̊ssbauer gamma ray line width \\
\hline $\mathrm{T}_{1}$ & Antiferromagnetic Néel transition temperature \\
\hline
\end{tabular}


Cavanagh, John Francis 3rd. Ph.D., Purdue University, June 1969. . Mössbauer Studies of the Transition Element Halides. Major Professor: James G. Mullen.

The Mỏssbauer effect in $\mathrm{Fe}^{57}$ has been used as a probe to study the temperature dependence of internal fields at $\mathrm{Fe}^{57}$ nuclei in $\mathrm{CoCl}_{2}$ and $\mathrm{CoF}_{2} \cdot \mathrm{CoCl}_{2}$, a metamagnet, exhibits both ferromagnetic and antiferromagnetic properties, while $\mathrm{CoF}_{2}$ is an antiferromagnet. Above the Néel temperature quantitative agreement was found in both magnitude and temperature dependence between the measured value of the electric field gradient at an $\mathrm{Fe}^{57}$ nucleus in a substitutional cobalt site in $\mathrm{CoCl}_{2}$, and that calculated by means of crystal field theory. The observed asymmetry in the quadrupole doublet lines of the spectrum is also explained on the basis of an anisotropic crystal binding. The Mössbauer source spectra of $\mathrm{CoF}_{2}$ was found to contain two quadrupole doublets. One doublet had the characteristic splitting and isomer shift of an $\mathrm{Fe}^{2+}$ ion while the other indicated an $\mathrm{Fe}^{3+}$ ion. The same sample when used as an absorber containing a small amount of $\mathrm{Fe}^{57}$ showed only the expected $\mathrm{Fe}^{2+}$ lines in its resonant absorption spectra. This observation, the first of its kind for a material with such a large characteristic Mo̊ssbauer temperature, suggests a local lattice distortion following $\mathrm{K}$-capture and the subsequent emission of an Auger electron, as the explanation of the origin of $\mathrm{Fe}^{3+}{ }_{i o n s}$ in the $\mathrm{CoF}_{2}$ 
source. The Néel temperature for $\mathrm{Fe}^{57}$ in $\mathrm{CoCl}_{2}$ and $\mathrm{CoF}_{2}$ are measured to be $25 \pm 2^{\circ} \mathrm{K}$ and $38 \neq 1^{\circ} \mathrm{K}$, respectively. The hyperfine field parameters below the Néel temperature were determined for $\mathrm{Fe}^{57}$ in both $\mathrm{CoCl}_{2}$ and $\mathrm{CoF}_{2}$. At $10^{\circ} \mathrm{K}$ the magnetic field at the nucleus was found to be $+49 \pm 2 \mathrm{kOe}$ in $\mathrm{CoCl}_{2}$. In $\mathrm{CoF}_{2}$ the magnitude of the magnetic hyperfine field was measured as $245 \pm 2 \mathrm{k} 0 \mathrm{e}$ at $11^{\circ} \mathrm{K}$. Assuming the sign of this field to be negative in the latter case, as found for other similar antiferromagnets, a positive sign is indicated for the component of the electric field gradient tensor $\mathrm{V}_{\mathrm{zz}}{ }^{\cdot}$ Although the Mössbauer spectra below $\mathrm{T}_{\mathrm{N}}$ are complex, it is possible to explain the experimental results in terms of a general magnetic dipole and electric quadrupole interaction. 


\section{INTRODUCTION}

The discovery by Mössbauer ${ }^{1}$ in 1958 of recoiless emission and absorption of gamma rays by nuclei bound in a crystalline lattice made nuclear resonance spectroscopy a practical laboratory tool. Previous to this time, resonant absorption or scattering experiments with gamma rays were difficult or impossible, because the recoil energy of the nucleus arising from momentum conservation in the emission or absorption process is typically several orders of magnitude greater than the linewidth of the emitted gamma ray. This recoil energy shift is more than sufficient to destroy the resonance conditions. Some success in resonant scattering experiments was achieved by introducing a Doppler shift to compensate for the recoil energy; either by means of a high speed rotor or thermal motion. Mössbauer, however, was able to show, by applying Lamb's theory ${ }^{2}$ of resonant neutron capture in solids to the case of gamma ray resonance in solids, that there is a finite probability that the nucleus will emit or absorb a gamma ray, without an accompanying change in the phonon states of the crystal. The recoil momentum is transferred to the entire solid and not just to an individual nucleus. In this case the recoil energy is reduced to a value which is completely negligible in comparison to the minimum gamma ray linewidth required by the uncertainty principle. Hence, resonant fluorescence of gamma rays can occur for nuclei bound in solids. 
For purposes of illustration, consider the most frequently used Mössbauer isotope $\mathrm{Fe}^{57}$. This is a natura11y occurring isotope with a relative abundance of 2.2 percent. It is also the daughter of $\mathrm{Co}^{57}$, whose decay scheme is shown in Fig. 1... The $14.4 \mathrm{keV}$ gamma ray emitted by $\mathrm{Fe}^{57}$ during the transition from the first excited state to the ground state is employed in Mös sbauer resonant absorption experiments. This gamma ray has a natural linewidth of about $10^{-8} \mathrm{eV}$ and can be Doppler shifted over a range of 100 linewidths by a relative velocity of only $1 \mathrm{~cm} / \mathrm{sec}$. In resonant absorption experiments the transmission of 14.4 kè gamma rays from a $\mathrm{Co}^{57}$ source, through an absorber containing $\mathrm{Fe}^{57}$, is measuredas a function of energy. The spectra 7 region may be scanned by moving the absorber relative to the source. This method of scanning $\dot{a}$ spectrum by Dopplex shifting has given rise to the practice of expressing energy shifts in units of velocity $(\mathrm{mm} / \mathrm{sec})$ in Mössbauer spectroscopy $\therefore$

The narrow 1inewidth of the gamma ray makes the resonant absorption spectrum very sensitive to changes in the nuclear energy levels. The Mössbauer effect permits the direct observation of the splitting of nuclear levels in crystalline fields. Since nuclei possess magnetic. dipole moments and electric quadrupole moments, their energy levels will be s.plit by interactions with magnetic fields and electric field. gradients, respectively. If the magnetic moment and the quadrupole moment of both the ground and excited states are known, the Mössbauer effect can be used to measure microscopic internal fields in solids. This application of the Mössbauex effect was recognized soon after. Mössbauer's discovery, and by 1961 the use of the Mössbauer effect in 


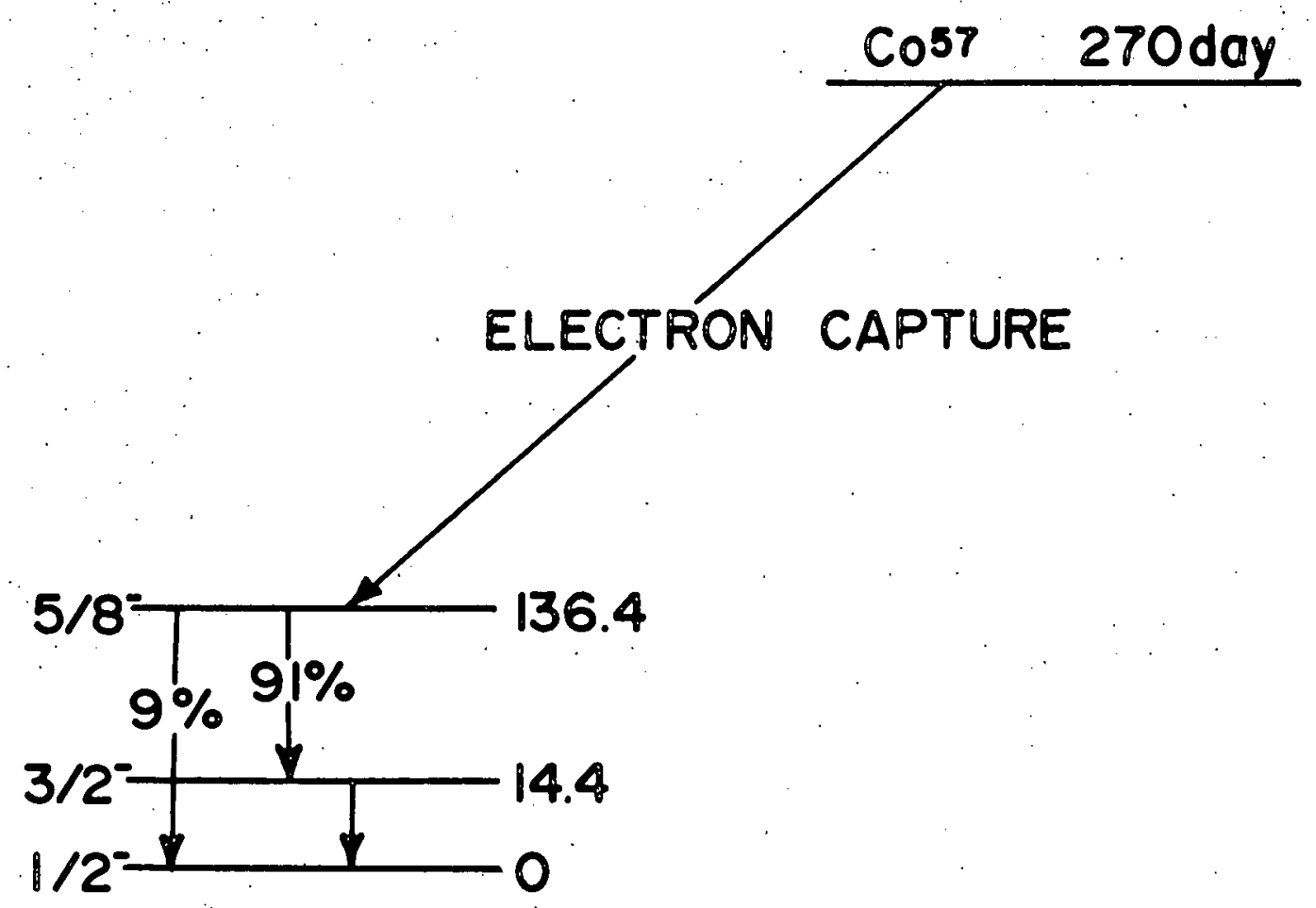

GROUND STATE

ENERGY = 0

$Q=0$

$\mu=+0.09024 \mathrm{~nm}$
FIRST EXCITED STATE

ENERGY $=14.4 \mathrm{keV}$

$T_{1 / 2}=9.77 \times 10^{-8} \mathrm{sec}$

$Q=0.28 b$

$\mu=-0.1547 \mathrm{~nm}$

Figure 1. Decay scheme of $\mathrm{Co}^{57}$. 
measuring internal fields in solids was already the subject of a review article. ${ }^{3}$

In addition to the two interactions mentioned above, there is also a monopole Coulomb interaction between the nucleus and the atomic electrons. This interaction produces a shift in the relative position of a resonant absorption line. This so called isomer shift is proportional to the total electron density at the nucleus and reflects the charge or valence state of the atom.

An electric field gradient at the $\mathrm{Fe}^{57}$ nucleus has no effect on the ground state since it is an $I=\frac{1}{2}$ state and, hence, has no quadrupole moment. The $I=3 / 2$ excited state, however, has a finite quadrupole moment and is split into two states by an electric field gradient. This causes the appearance of a doublet in the Mössbauer spectrum, whose. separation is a function of the electric field gradient, as shown in Fig. 2a. A magnetic field will split both the ground and excited states of $\mathrm{Fe}^{57}$ into two and four states, respectively. This splitting of the nuclear levels and the selection rule $\Delta \mathrm{m}= \pm 1,0$ lead to a Mossbauer spectrum consisting of six lines whose relative spacing is proportional to the magnetic field: Fig. 2b illustrates this schematically. In the case of a combined magnetic field and electric field gradient at the nucleus, $m$ will, in general, no longer be a good quantum number. This leads to the presence of as many as eight lines in the Mössbauer pattern as shown in Fig. 2c. Although the pattern may be quite complex, methods exist $^{4-7}$ for determining the value of both the magnetic field and the electric field gradient from the relative position and intensities of the lines. 

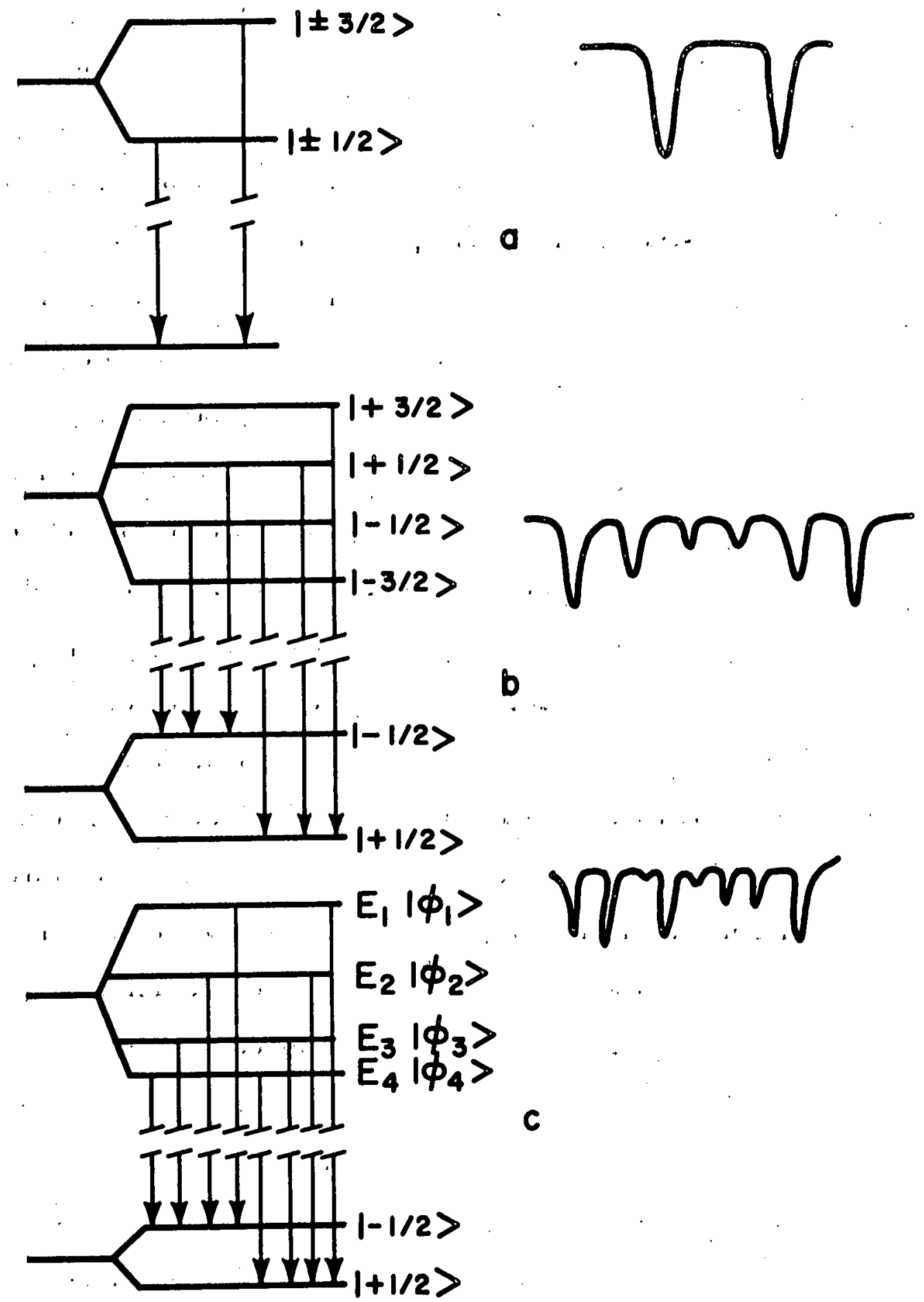

Figure 2. Nuclear level splitting and resultant Mössbauer spectra for $\mathrm{Fe}^{57}$. a) electric quadrupole interaction, b) magnetic dipole interaction, c) combined electric quadrupole and magnetic dipole interaction. 
The Mössbauer effect has been used to study internal fields in a great many iron compounds ${ }^{8}$. There has been very little use of the Mössbauer effect in the study of cobalt compounds probably because of expected complications resulting from possible Auger aftereffects. Wertheim ${ }^{9}$ observed two distinct spectra for CoO containing $\mathrm{Co}^{57}$. These spectra had isomer shifts corresponding to $\mathrm{Fe}^{2+}$ and $\mathrm{Fe}^{3+}$ ions. Normally $\mathrm{Fe}^{2+}$ ions would be expected for iron in cobaltous oxide. Wertheim attributed the observation of a higher charge state to a slow relaxation to electronic equilibrium, following k-capture in the radioactive decay of $\mathrm{Co}^{57}$. The hole in the $\mathrm{K}$-she11, which occurs after the $\mathrm{K}$-capture results in an excitation, which is dissapated by both $x$-ray emission and the ejection of Auger electrons. In elements with a low atomic number such as iron $(Z=26)$ the latter process is more probable by more than a factor of two. ${ }^{10}$ This process of Auger emission leaves the atom in a higher charge state following the radioactive decay. If, as Wertheim proposed, the time required to reach electronic equilibrium is of the same order, as the mean life of the first excited state of the $\mathrm{Fe}^{57}$ nucleus, then gamma rays will be emitted while the ions are in both the $\mathrm{Fe}^{3+}$ and $\mathrm{Fe}^{2+}$ states. More detailed experiments, 11, 12, however, show conclusively that Auger aftereffects are not the origin of the two charge states observed in CoO. Similar multiple charge states have been observed in the hydrated $\operatorname{CoC}_{2}$ compounds, $^{13}$ and Auger aftereffects were originally proposed as their origin. A systematic study of the hydrated $\mathrm{CoCl}_{2}$ compounds, ${ }^{12}$ however, suggests that a loca 1 lattice rearrangement, caused by effects associated with previous nuclear events, can most easily explain the multiple charge states in the 
Mössbauer spectra of these compounds. Nevertheless, the study of nonmetallic cobalt compounds has been avoided since Wertheim's interpretation was assumed to hold in general and thus complicate the interpretation of the Mössbauer spectra for such compounds.

The object of this research was to use the Mössbauer effect to study anhydrous cobaltous chloride and anhydrous cobaltous fluoride. We sought to gain further insight in the formation of multiple charge states, and to investigate the intemal fields in these materials. Both of these compounds exhibit unusual magnetic properties and it was expected that the Mössbauer effect would yield new information concerning their magnetic behavior.

Anhydrous cobaltous chloride has been classified as a member of a class of materials known as metamagnets. These materials exhibit both ferromagnetic and antiferromagnetic properties. The bulk susceptability of $\mathrm{CoCl}_{2}$ obeys the Curie-Weiss law for a ferromagnet. $\mathrm{X}=\mathrm{C} /\left(\mathrm{T}-\mathrm{T}{ }_{c}\right)$, in the paramagnetic region. ${ }^{14}$ The specific heat, however, shows the $\lambda$-type anomaly characteristic of an antiferromagnet. ${ }^{15}$ Low field susceptability measurements show a maximum in the curve which occurs at the same temperature as the specific heat anomaly. When these same measurements are made with higher external fields, on the other hand, the susceptability exhibits saturation effects characteristic of a ferromagnet, although neither spontaneous magnetization nor hysteresis effects have been observed. $\mathrm{CoCl}_{2}$ has the $\mathrm{CdCl}_{2}$ rhombohedral structure ${ }^{16} a_{0}=6.16 \mathrm{~A}$, $\alpha=33^{\circ} 26^{\prime}$ ), which may also be viewed as hexagonal layers of cobalt atoms separated by two hexagonal layers of chlorine atoms. Landau 17 proposed a model of the magnetic structure of this compound which 
postulated an intralayer ferromagnetic interaction and a weak interlayer antiferromagnetic interaction. The high field behavior could then be explained by the external field overcoming the antiferromagnetic interaction and bringing about a parallel alignment of the spins. The actual magnetic structure, $\cdots$ as determined by neutron diffraction studies, ${ }^{18}$ confirms this model.

A. Mös sbauer study of anhydrous $\mathrm{FeCl}_{2}$ has been made. ${ }^{19}$. This compound is isomorphous with $\operatorname{CoCl}_{2}$ and is also a metamagnet. An unusual feature. of the Moossbauer spectrum of $\mathrm{FeCl}_{2}$ is the absence of any resolvable magnetic field at the nucleus in the antiferromagnetic temperature range. This was attributed to a fortuitous cancellation to about one part in $10^{3}$ of the spin-orbit, Fermi contact and dipole-dipole terms which make up the internal field at the nucleus. This cancellation effect has recently been confirmed by independent investigators. ${ }^{20}$ The quadrupole interaction in the paramagnetic region is also very small compared with many other $\mathrm{Fe}^{2+}$ compounds, and it has a relatively large temperature dependence. Ito and ono were able to explain the paramagnetic quadrupole spectra using crystal field theory.

The Mössbauer spectra of cobalt compounds are made by examining an iron impurity in a cobalt lattice site. An analysis of the paramagnetic quadrupole splitting for $\mathrm{Fe}^{57}$ in $\mathrm{CoCl}_{2}$ has been made here using crystal field theory. The results agree quite well with the experimental data. The $\mathrm{CoCl}_{2}$ spectra in the paramagnetic region, i.e., above the Née1 temperature, show an asymmetry in the relative intensity of the two lines of the doublet. The: asymmetry increases monotonically with tempera-ture and is believed to be an example of the Goldanski-Karyagin 
effect $^{21,22}$, which is related to crystallographic anisotropy in the binding of an $\mathrm{Fe}^{57}$ ion.

Below the Néel temperature the $\mathrm{CoCl}_{2}$ Mössbauer spectra are very complex but appear to indicate that there is a magnetic field at the nucleus. Unfortunately, the spectra are not well resolved in this region, which introduces a large uncertainty into the estimates of the hyperfine field strength.

Anhydrous cobaltous fluoride has the $\mathrm{SnO}_{2}$ rutile crystal structure 23 $\left.a_{0}=4.69 \mathrm{~A}, c_{0}=3.19 \mathrm{~A}\right)$. The lack of a well defined "kink" in its susceptability curve caused Bezette ${ }^{24}$ to conclude that $\mathrm{CoF}_{2}$ was not an antiferromagnet in contrast with the isomorphous compounds $\mathrm{FeF}_{2}$ and $\mathrm{MnF}_{2}$. This conclusion, however, was contradicted a few years later by neutron diffraction studies ${ }^{25}$ and specific heat measurements, ${ }^{26}$ which confirmed the existence of antiferromagnetic ordering in $\mathrm{CoF}_{2} \cdot$ Even these two experiments were not in complete agreement. The former gave a Néel temperature of $50^{\circ} \mathrm{K}$ while the latter yielded a value of $37.7^{\circ} \mathrm{K}$.

The hyperfine constants were calculated for both $\mathrm{CoCl}_{2}$ and $\mathrm{CoF}_{2}$ and are found to be in reasonable agreement with other similar materials. Our determination of the Nél temperature, $\mathrm{T}_{\mathrm{N}}$, for both salts is dis-. cussed and compared with the specific heat and neutron scattering data. Experiments were done on both $\mathrm{Co}^{57}$ doped sources and $\mathrm{Fe}^{57}$ doped absorbers for both $\operatorname{CoCl}_{2}$ and $\mathrm{CoF}_{2}$. This was particularly useful for the latter case where there is not a simple symmetry in the two experiments, and will be shown to help answer questions concerning the origin of the $\mathrm{Fe}^{3+}$ resonance found in $\mathrm{CoF}_{2}$. 


\section{THEORY}

\section{A. Recoil-free-Fraction}

The probability that a nucleus will emit or absorb a gamma ray without recoil is known as the recoil-free fraction, $f$, and is given by the square of the matrix element:

$$
f=\left|\cdot\left\langle n\left|e^{i \stackrel{x}{x} \cdot \vec{r}}\right| n\right\rangle\right|^{2}
$$

where $|n\rangle$ is the wave function describing the lattice; $\vec{r}$ is displacement vector of the atomic motion; and $\vec{x}$ is the momentum vector of the gamma ray divided by $\hbar$ : For a solid whose interatomic forces are harmonic, this matrix element squared can be written ${ }^{27}$

$$
f=\exp \left(-x^{2}\left(u^{2}\right\rangle\right)
$$

where $\left\langle u^{2}\right\rangle$ is the mean square displacement of the atom in the direction of motion of the gamma ray. For a Debye solid $x^{2}\left\langle u^{2}\right\rangle$ has a value given by the expression 28

$$
x^{2}\left\langle u^{2}\right\rangle=\frac{6 R}{k \theta}\left[\frac{1}{4}+\left(\frac{T}{\theta}\right)^{2} \int_{0}^{\theta / T} \frac{x d x}{e^{x}-1}\right],
$$

where $R$. is the free nucleus recoil energy; $\theta$ is a characteristic temperature, $k$ is Boltzmann's constant and $T$ is the temperature of the solid. 
A1though, even:as an approximation, the Debye mode1 is applicable only to monatomic lattices with a Debye phonon spectrum, it can be successfully used in calculating the recoil-free fraction of more complex crystals if the Debye temperature $\theta$, as determined from the specific heat, is replaced by a characteristic Mossbauer temperature $\theta_{m}$, in the above equation:. This $\theta_{\mathrm{m}}$ may not have a close relation to $\theta$ if the $10 \mathrm{cal}$ binding of the Mossbauer ion is quite different than host ions in the solid.

The recoil-free fraction is determined experimentally by measuring the area under the absorption curve. It can be shown ${ }^{29}$ that this area, (S), is proportional to the recoil-free fraction of the source. The area is also proportional to the recoil-free fraction of the absorber in the case of thin absorbers. Thus, for a fixed absorber, the area under the absorption curve as a function of source temperature can be written

$$
S=\operatorname{Cf}(T)
$$

where $\mathrm{C}$ is the constant of proportionality. Thus, from the relation

$$
S=C \exp \left[-x^{2}\left\langle u^{2}\right\rangle\right]
$$

we get in the Debye approximation,

$$
\ln S(T)=\ln C-\frac{6 R}{k \theta_{m}}\left[\frac{1}{4}+\left(\frac{T}{\theta_{m}}\right)^{2} \cdot \int_{0}^{\theta_{m} / T} \cdot \frac{x d x}{e^{x}-1}\right] .
$$

In the low temperature limit $\mathrm{T} . \ll \theta_{\mathrm{m}}$, we can make the approximation

$$
\int_{0}^{\infty} \frac{x d x}{e^{x}-1}=\frac{\pi^{2}}{6}
$$


giving,

$$
\begin{aligned}
& f \cong \exp \left[-\frac{3}{2} \frac{R}{k \theta_{m}}\left(1+\frac{2 \pi^{2}}{3} \frac{T^{2}}{\theta_{D}^{2}}\right)\right], \\
& 1 n S \cong 1 n C-\frac{3 R}{2 k \theta_{m}}-\frac{\pi^{2} R}{k \theta_{m}^{3}} T^{2} .
\end{aligned}
$$

In the high temperature case where the lattice energy approaches its classical 1imit $\left(\mathrm{T} .>\theta_{\mathrm{m}} / 2\right)$,

$$
f \cong \exp \left[-\frac{6 R}{k \theta_{m}^{2}} \mathrm{~T}\right]
$$

and

$$
\ln S=\ln \mathrm{C}-\frac{6 \mathrm{R}}{\mathrm{k} \theta_{\mathrm{m}}^{2}} \mathrm{~T} .
$$

By using these relations we can directly find the Mössbauer characteristic temperature, $\theta_{\mathrm{m}}$, from the temperature dependence of the area under the resonance absorption curves.

B. Crystal Perturbations of Nuclear Levels

There are three types of interactions between the nucleus and external fields which are observable with the Mơssbauer effect. The first is a Coulomb interaction between the nucleus and atomic electrons. The strength of this interaction is a function of the charge radius of the nucleus and the atomic electron density at the nucleus. Since the ground and excited states have different charge radii, there is a net 
shift in the energy difference of the two states. If the chemical environment of the source and absorber are such that there is a difference in the atomic electron density at the nucleus, there will then be a net shift in the position of the maximum absorption, which in the usual approximations is given by

$$
\delta=\frac{3}{5} \pi z e^{2} \cdot\left[R_{g}^{2}-R_{e}^{2}\right]\left[\psi(0)_{a}^{2}-\psi(0)_{s}^{2}\right],
$$

where $\mathrm{Z}$ is the atomic number of the nucleus, $\mathrm{R}_{g}$ and $\mathrm{R}_{\mathrm{e}}$ are the charge radii of the ground and excited states, and $\left|\psi(0)_{a}\right|^{2}$ and $\left|\psi(0)_{s}\right|^{2}$ are proportional to the total electron densities evaluated at the nucleus for the absorber and source. This shift in the energy center of the spectrum is termed the chemical or isomer shift. ${ }^{30}$ The isomer shift is useful in determining the charge or valence state of the atom whose nucleus takes part in the resonant emission or absorption process. In the case of $\mathrm{Fe}^{57}$ below room temperature, for example, an $\mathrm{Fe}^{2+}$ ion has an isomer shift of about $1.5 \mathrm{~mm} / \mathrm{sec}$ relative to metalic sources or absorbers, while an $\mathrm{Fe}^{3+}$ ion has a shift of on $1 \mathrm{y}$ about $0.5 \mathrm{~mm} / \mathrm{sec}$.

The isomer shift is found to be temperature dependent. In addition to the isomer shift another temperature dependent shift is the Josephson shift. 31 Two approaches can be used to explain this temperature dependence. Pound and Rebka ${ }^{32}$ view it in terms of a second order Doppler shift while' Josephson ${ }^{31}$ treats it in terms of the mass change of the nucleus after gamma ray emission. The methods are equivalent and yield the same results. In the standard Mơssbauer units of velocity, this relative shift per ${ }^{\circ} \mathrm{K}$ can be written as $\Delta V=\left(C_{p} / 2 c\right)$, where $C_{p}$ is the 
lattice specific heat and $c$ is the speed of light in free space. In the case of $\mathrm{Fe}^{57}$, the shift is of the order of $0.2 \mathrm{~mm} / \mathrm{sec}$ for a $300^{\circ} \mathrm{K}$ temperature difference bẹtween source and absorber.

A non-zero electric field gradient at the $\mathrm{Fe}^{57}$ nucleus will split the $I=3 / 2$ excited state into two energy states. Since $I=1 / 2$ ground state has no quadrupole moment, it is not perturbed by an electric field gradient. The interaction may be written 33

$$
\mathcal{F}=\stackrel{Q}{\mathrm{~S}} \cdot(\nabla \mathrm{DE})
$$

where $Q$ is the electric quadrupole moment tensor of the nucleus and, $\nabla E$ is the electric field gradient tensor. By choosing the coordinate axes such that $\underset{\sim E}{ }$ is diagonal, and by using the fact that the three components of the electric field gradient tensor must obey LaPlaces' equation, $\mathrm{V}_{\mathrm{xx}}+\mathrm{V}_{\mathrm{yy}}+\mathrm{V}_{\mathrm{zz}}=0$, one can express the electric field gradient in terms of its z-component $Q=v_{z z} / e$, and an asymmetry parameter $\eta=\left(v_{x x}-V_{y y}\right) /$ $\mathrm{V}_{z z}$, such that the quadrupole interaction can be written in the form;

$$
\mathcal{H C}=\frac{\mathrm{e}^{2} \mathrm{qQ}}{2 I(2 I-1)}\left[3 \mathrm{I}_{z}{ }^{2}-\vec{I}^{2}+\eta\left(I_{x}{ }^{2}-I_{y}{ }^{2}\right)\right],
$$

where $Q$ is the component of the electric quadrupole moment $Q_{z z}$, and $\vec{I}^{2}$ is the square of the total angular momentum operator. For the $I \pm 3 / 2$ excited state of $\mathrm{Fe}^{57}$, this Hamiltonian has eigenvalues

$$
: E= \pm \frac{e^{2} g Q}{4}\left(1+\frac{\eta^{2}}{3}\right)^{\frac{3}{2}}
$$

with eigenvectors corresponding to linear combinations of the states $|3 / 2, \pm 3 / 2\rangle$ and $\left|3 / 2, \pm \frac{3}{2}\right\rangle$. This splitting leads to a doublet in 
a Mo̊ssbauer spectrum. The two lines are of equal intensity for powder specimens in which the recoil-free fraction is isotropic. If there is an anisotropy in the recoil-free fraction, there will be an asymetry in the doublet. This is the Goldanski-Karyagin effect which will be discussed in section $C$.

A pure magnetic field at the nucleus interacts with the magnetic moment of both the ground and excited states as described by the Hamiltonians:

$$
\mathcal{F C}_{e}=-g_{1} \mu_{N} \vec{H} \cdot \vec{I} \text {, and } \mathcal{F C}_{g}=-g_{0} \mu_{N} \vec{H} \cdot \vec{I} \text {. }
$$

where $g_{0}$ and $g_{1}$ are the nuclear gyromgnetic ratios of the ground and excited states respectively; $\mu_{\mathrm{N}}$ is the nuclear Bohr magnetron; and $\overrightarrow{\mathrm{H}}$ is the magnetic field strength. The eigenvalues of this Hamiltonian are given by the expression,

$$
E=-g \mu_{\mathrm{N}} \mathrm{mH}
$$

where $\mathrm{m}$ is the magnetic quantum number. Thus, for the case of $\mathrm{Fe}^{57}$ considered here, the excited. state is split into four states and the ground state is split into two states. The selection rule for magnetic dipole transitions, $(\Delta m= \pm 1,0)$, restricts the transitions between excited and ground state sublevels such that six lines appear in the magnetically sp1it Moossbauer spectrum. If the two outer peaks of this pattern are separated by an anount $\Delta V$, then the magnitude of the magnetic field at the nucleus is given by the relationship:

$$
H=\Delta V /\left|\left(3 g_{1}-g_{0}\right)\right| \mu_{N}
$$


In the general case of a combined magnetic field and electric field gradient at the nucleus, the Hamiltonians describing the interactions for both the ground and excited state can be written as:

$$
J_{e}=-g_{1} \mu_{N} H I_{z}+\frac{e^{2} q Q}{2 I(2 I-1)}\left[3 I^{2},-I^{2}+\eta\left(I_{x}^{2}-I_{y}^{2}\right)\right]
$$

and

$$
\mathcal{F}_{\mathrm{g}}=-\mathrm{g}_{\mathrm{O}} \mu_{\mathrm{N}} \mathrm{HI} \mathrm{I}_{\mathrm{z}} \text {, }
$$

where the $z$-axis is parallel to the magnetic field and the primed coordinates are the principal axes of the electric field gradient. If $\theta$ and $\phi$ are the polar and azimuthal angles describing the relative orientation of the primed and unprimed coordinate systems, one can apply a unitary transformation to the exicted state Hamiltonian which rotates the primed system into the unprimed system. In this way the ground state Hamiltonian remains diagonal. After performing this rotation the components of the Hamiltonian matrix can be constructed with the standard raising and lowering operator formalism. In units of $\left|g_{1} \mu_{N} H\right|$ this matrix has the form

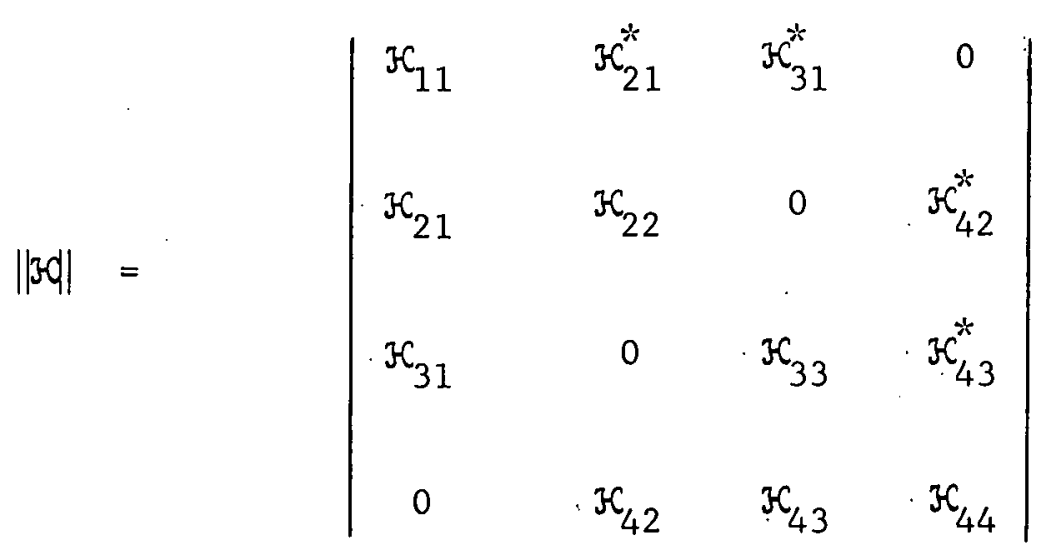


If we define the quantity, $\quad P=\frac{e^{2} q Q}{6 g_{1} \mu_{N} H}$, then:

$$
\begin{aligned}
& { }^{3-C_{11}}=3 / 2+(9 / 8) \mathrm{P}\left(3 \cos ^{2} \theta+\eta \sin ^{2} \theta \cdot \cos 2 \phi\right)-(15 / 8) \mathrm{P}+ \\
& (3 / 8) P(3-\eta \cos 2 \phi) \sin ^{2} \theta \text {, } \\
& { }^{-} C_{22}=1 / 2+(7 / 8) P(3-\eta \cos 2 \phi) \sin ^{2} \theta+(1 / 8) P\left(3 \cos ^{2} \theta+\eta \sin ^{2} \theta \cos 2 \phi\right) \\
& \text { - }(15 / 8) \mathrm{P} \text {, } \\
& \mathfrak{F}_{33}=\mathfrak{H}_{22}-1 \text {, } \\
& 3 C_{44}=3 C_{11}-3 \\
& \left.3_{21}=-3_{43}=\sqrt{\frac{3}{2}} p \cdot-3 \sin \theta \cos \theta+h \sin \theta(\cos \theta \cos 2 \phi-i \sin 2 \phi)\right],
\end{aligned}
$$

and

$$
H_{31}=\mathfrak{H C}_{42}=\sqrt{\frac{3}{4}} \mathrm{P}\left[3 \sin ^{2} \theta+\eta \cos 2 \phi\left(1+\cos ^{2} \theta\right)-2 i \cdot \eta \cos \theta \sin 2 \phi\right] \text { : }
$$

There is no general analytical solution to this eigenvalue problem for the excited state. Since the ground state is diagonal for the present choice of axes, the eigenvalues of the ground state can be found in a straightforward way and are identical to those in the pure magnetic field case: If the values of the parameters $H, q, \eta, \theta$, and $\phi$ are known, numerical solutions for the eigenvalues and eigenvectors of the excited state can be found with the use of computer programs for matrix diagonalization. These solutions and the resulting Mo̊ssbauer spectrum will have the form shown in Table 1 . Since $\mathrm{m}$. is no longer a good quantum 
TABLE 1.

General form of the solution for the first excited state of $\mathrm{Fe}^{57}$. under a combined magnetic dipole and electric quadrupole interaction and the positions and intensities of the relative Mössbauer lines.

ENERGY

\section{EIGENVECTORS}

$\begin{array}{ll}E_{1} & a_{11}|-3 / 2\rangle+a_{21}|-1 / 2\rangle+a_{31}|1 / 2\rangle+a_{41}|3 / 2\rangle \\ E_{2} & a_{12}|-3 / 2\rangle+a_{22}|-1 / 2\rangle+a_{32}|1 / 2\rangle+a_{42}|3 / 2\rangle \\ E_{3} & a_{13}|-3 / 2\rangle+a_{23}|-1 / 2\rangle+a_{33}|1 / 2\rangle+a_{43}|3 / 2\rangle \\ E_{4} & a_{14}|-3 / 2\rangle+a_{24}|-1 / 2\rangle+a_{34}|1 / 2\rangle+a_{44}|3 / 2\rangle \\ & E_{G}=g_{0} H_{N} H^{H / 2}\end{array}$

LINE POSITION

RELATIVE INTENSITY

$$
\begin{array}{r}
E_{1}+E_{G} \\
E_{2}+E_{G} \\
E_{1}+E_{G} \\
E_{3}+E_{G} \\
E_{2}-E_{G} \\
E_{4}+E_{G} \\
E_{3}-E_{G} \\
E_{4}-E_{G}
\end{array}
$$$$
3\left|a_{11}\right|^{2}+2\left|a_{21}\right|^{2}+\left|a_{31}\right|^{2}
$$$$
3\left|a_{12}\right|^{2}+2\left|a_{22}\right|^{2}+\left|a_{32}\right|^{2}
$$$$
\left|a_{21}\right|^{2}+2\left|a_{31}\right|^{2}+3\left|a_{41}\right|^{2}
$$$$
3\left|a_{13}\right|^{2}+2\left|a_{23}\right|^{2}+\left|a_{33}\right|^{2}
$$$$
\left|a_{22}\right|^{2}+2\left|a_{32}\right|^{2}+3\left|a_{42}\right|^{2}
$$$$
3\left|a_{14}\right|^{2}+2\left|a_{24}\right|^{2}+\left|a_{34}\right|^{2}
$$$$
\left|a_{23}\right|^{2}+2\left|a_{33}\right|^{2}+3\left|a_{43}\right|^{2}
$$$$
\left|a_{24}\right|^{2}+2\left|a_{34}\right|^{2}+3\left|a_{44}\right|^{2}
$$ 
number for the excited state, transitions can occur between al1 sublevels of both the excited and ground states. This leads to the appearance of eight lines in the hyperfine spectrum.

Karyagin ${ }^{6}$ has developed a method for determining the quantities $H$ and $1 / 2\left|e^{2} \mathrm{qQ}\right|\left(1+\eta^{2} / 3\right)^{\frac{1}{2}}$ precisely, and $\eta, \theta$ and $\phi$ approximately from the experimentally measured line positions. These estimates and the computer generated numerical solutions to the above eigenvalue problem can be used in a curve fitting procedure to refine the estimates of the five parameters characterizing the internal fields at the nucleus. The accuracy of this method depends on how well the eight lines in the Mössbauer spectrum can be resolved and their peak positions determined.

Karyagin shows that the eigenvalues of the Hamiltonian matrix can be determined from the peak positions in the spectrum. Once these eigenvalues have been found it is possible to solve the secular equation of the perturbation problem for the quantities $H$ and $\frac{1}{2}\left|e^{2} q Q\right| \sqrt{1+\eta^{2} / 3}$ exactly, and obtain approximate values for $\eta, \theta$, and $\phi$. If one writes the secular determinant in the form:

$$
\frac{3}{2}\left|\begin{array}{cccc}
\mathrm{B}+\mathrm{C}-\lambda & \mathrm{D} & \mathrm{E} & 0 \\
\mathrm{D}^{*} & \frac{\mathrm{B}}{3}-\mathrm{C}-\lambda & 0 & \therefore \mathrm{E} \\
\mathrm{E}^{*} & 0 & -\frac{\mathrm{B}}{3}-\mathrm{C}-\lambda^{\circ} & -\mathrm{D} \\
0 & \because \mathrm{E}^{*} & -\mathrm{D}^{*} & -\mathrm{B}+\mathrm{C}-\lambda
\end{array}\right|
$$

where $B=g_{1} \mu_{N} H$, the algebraic expressions are simplified.

In the process of finding the eigenvalues of the excited state 
from the line positions one also obtains the 'ground state splitting: $\Delta E_{G}=g_{0} \mu_{N} H . \quad$ Thus

$$
\ldots \quad \quad \therefore \quad \mathrm{B}^{2}=\left(\frac{\mathrm{g}_{1}}{\mathrm{~g}_{0}} \cdot \mathrm{E}_{\mathrm{G}}\right)^{2}
$$

The secular determinant can also be written in the form

$$
\operatorname{det}|H-\lambda I|=\left(\lambda-E_{1}\right)\left(\lambda-E_{2}\right)\left(\lambda-E_{3}\right)\left(\lambda-E_{4}\right)=0
$$

where $E_{i}(i=1,2,3 ; 4)$ is an eigenvalue of the excited state. If both the determinant and polynomial are expanded and coefficients of the same power of $\lambda$ are equated, one can obtain equations for the matrix elements. With the introduction of the defined quantities:

$$
\begin{aligned}
\alpha & =\sum_{\mathrm{k}} \mathrm{E}^{2}, \\
\mu & =\sum_{\mathrm{k}} \mathrm{E}_{\mathrm{k}}^{3}, \cdots \\
\gamma & =\mathrm{E}_{1} \mathrm{E}_{2} \mathrm{E}_{3} \mathrm{E}_{4},
\end{aligned}
$$

coupled with the relationship $E_{1}+E_{2}+E_{3}+E_{4}=0$ these equations are:

$$
\begin{gathered}
C=\frac{\mu}{18 B^{2}} \cdots \\
|E|^{2}+|D|^{2}=\frac{\alpha-5 B^{2}}{9}-C^{2} \\
|E|^{2}-|D|^{2}=\frac{3}{2 B^{2}}\left[\frac{16}{81} \gamma-\left(\frac{\alpha-5 B^{2}}{9}\right)^{2}+\frac{10}{9} B^{2} \cdot C^{2}-\frac{B^{4}}{9}\right] .
\end{gathered}
$$


It then follows from the definition of these matrix elements that

$$
e_{\mathrm{q}}=\frac{1}{2}\left|\mathrm{e}^{2} \mathrm{qQ}\right|\left(1+\eta^{2} / 3\right)^{\frac{2}{2}}=\left(\alpha-5 \mathrm{~B}^{2}\right)^{\frac{2}{2}}
$$

Thus exact expressions can be found both for the magnitude of the magnetic field and quadrupole interaction. It is not possible to find such explicit expressions for $\theta, \phi$, and $\eta$. However, if we define the quantity $\mathrm{X}$ as :

$$
\mathrm{x}=\frac{1}{6} \mathrm{e}^{2} \mathrm{qQ}=\frac{\varepsilon_{\mathrm{q}}}{3}\left(1+\frac{\eta^{2}}{3}\right)^{\frac{2}{2}}
$$

whose absolute value lies in the range $\frac{6 q}{2 \sqrt{3}} \leq x \leq \frac{6 q}{3}$; since $0 \leq \ddot{\eta} \leq 1$, and the related quantities $\mathrm{Y}$ and $\mathrm{Z}$ given by

$$
\begin{gathered}
Y=2(X-C) / X \text {, and } \\
\therefore Z=Y\left[(2 X+C)^{2}-3|E|^{2}\right] /\left[4(X-C)(2 X+C)-3|D|^{2}\right],
\end{gathered}
$$

then,

$$
\begin{aligned}
& \sin ^{2} \theta=\frac{Y}{Y+Z}, \\
& \eta=\frac{1}{3}\left(\frac{\epsilon}{X}\right)^{2}-3,
\end{aligned}
$$

and,

$$
\cos 2 \phi=(3-\mathrm{Y}-\mathrm{Z}) / \eta
$$




\section{Go1danski-Karyagin Effect}

$\therefore$ An explanation for the asymmetry in Mössbauer quadrupole doublets first observed by Goldanski ${ }^{21}$ has been given by Karyagin ${ }^{22}$.. If the recoil-free fraction has an angular dependence due to an anisotropy in the lattice binding, the ratio of the intensity of the doublets in case of an axiálly symmetric electric field gradient can be written:

$$
\frac{I_{\pi}}{I_{\sigma}}=\frac{\int_{0}^{\pi} i_{\pi}(\theta) f(\theta) \sin \theta d \theta}{\int_{0}^{\pi} i_{\sigma}(\theta) f(\theta) \sin \theta d \theta},
$$

where $I_{\pi}$ and $I_{\sigma}$ are the intensities of the $\mid 3 / 2, \pm 3 / 2>$ to $\left|\frac{1}{2}, \pm \frac{I}{2}\right\rangle$ and the $\mid 3 / 2, \pm \frac{3}{2} \gg$ to $\left|\frac{3}{2}, \pm \frac{3}{2}\right\rangle$ transitions, respectively. . The subscripts used are standard spectroscopic notation. The terms $i_{\pi}(\theta)$ and $i_{\sigma}(\theta)$ are the corresponding angular parts of the multipole radiation probabilities for the two transitions, i.e.,

$$
\begin{aligned}
& i_{\pi}=\text { const. }\left(1+\cos ^{2} \theta\right), \\
& i_{\sigma}=\operatorname{const} \cdot\left(5 / 3-\cos ^{2} \theta\right) .
\end{aligned}
$$

In solids having harmonic forces the recoil-free fraction is given by the expression :quoted in section $A$,

$$
f=\exp \left[-\dot{x}^{2}\left\langle u^{2}\right\rangle\right]
$$

The displacement of the nucleus in the direction of the gamma ray, 
$u$, is defined.by

$$
u=\frac{\overrightarrow{\underline{u}} \cdot \vec{r}}{|k|}
$$

where $\vec{r}$ is the displacement vector of the nucleus from its equilibrium position in the lattice. If the crystal has axial symetry about the z-axis as in the case of CoC $\mathrm{I}_{2}$, then

$$
\vec{r}=\hat{i} x+\hat{k} z
$$

with $\mathbf{z}$ being the component of the displacement parallel to axis of symmetry and $x$ being a component perpendicular to this axis. $\hat{i}$ and $\hat{\hat{k}}$ are unit vectors in the directions. Also we have

$$
\frac{\vec{u}}{|\dot{x}|}=\hat{i} \cdot \sin \theta+\hat{k} \cos \theta,
$$

where $\theta$ is measured with respect to the $z$-axis. Then, $u=x \sin \theta+z$ $\cos \theta$ and $u^{2}=x^{2} \sin ^{2} \theta+2 x z \sin \theta \cos \theta+z^{2} \cos ^{2} \theta$. Thus $\left\langle u^{2}\right\rangle=\left\langle x^{2}\right\rangle$ $\sin ^{2} \theta+\left\langle z^{2}\right\rangle \cos ^{2} \theta$ since $\langle x\rangle=\langle z\rangle=0$. Using the Pythagorean identity,

$$
\cos ^{2} \theta+\sin ^{2} \theta=1
$$

$\therefore$ one can then write the axially symetric recoil-free fraction

$$
f=\exp \left\{-\mu^{2} \cdot\left[\left(\left\langle z^{2}\right\rangle-\left\langle x^{2}\right\rangle\right) \cos ^{2} \theta+\left\langle x^{2}\right\rangle\right]\right\}
$$

If we now let $\varepsilon^{2}=\left|u^{2}\left(\left\langle z^{2}\right\rangle-\left\langle x^{2}\right\rangle\right)\right|$, the ratio of the intensities of the doublet'can be written 


$$
\frac{I_{\pi}}{I_{\sigma}}=\frac{\int_{-1}^{1}\left(1+\cos ^{2} \theta\right) \exp \left[-\varepsilon^{2} \cos ^{2} \theta-x^{2}\left\langle x^{2}\right\rangle\right] d(\cos \theta)}{\int_{-1}^{1}\left(\frac{5}{3}-\cos ^{2} \theta\right) \exp \left[-\varepsilon^{2} \cos ^{2} \theta-x^{2}\left\langle x^{2}\right\rangle\right] d(\cos \theta)}
$$

Letting $\cos \theta=t$, and factoring the common term $\exp \left(-\dot{x}^{2}\left\langle x^{2}\right\rangle\right)$, gives

$$
\frac{I_{\pi}}{I_{\sigma}}=\frac{\int_{-1}^{1}\left(1+t^{2}\right) \exp \left(-\epsilon^{2} t^{2}\right) d t}{\int_{-1}^{1}\left(\frac{5}{3}-t^{2}\right) \exp \left(-\epsilon^{2} t^{2}\right) d t} .
$$

Two cases can be distinguished:

$$
\epsilon^{2} \leq 0 ;\left\langle x^{2}\right\rangle \geq\left\langle z^{2}\right\rangle
$$

and

$$
\varepsilon^{2} \geq 0 ;\left\langle x^{2}\right\rangle \leq\left\langle z^{2}\right\rangle
$$

Performing the integration we obtain the results:

$$
\frac{I_{\pi}}{I_{\sigma}}=\frac{\left(6 \varepsilon^{2}-3\right) F(\varepsilon)+3|\varepsilon| e^{\varepsilon^{2}}}{\left(10 \varepsilon^{2}+3\right) F(\epsilon)-3|\epsilon| e^{\epsilon^{2}}},
$$

when $\sigma^{2} \leq 0$, and where

$$
F(\varepsilon)=\int_{0}^{|\epsilon|} e^{t^{2}} d t .
$$




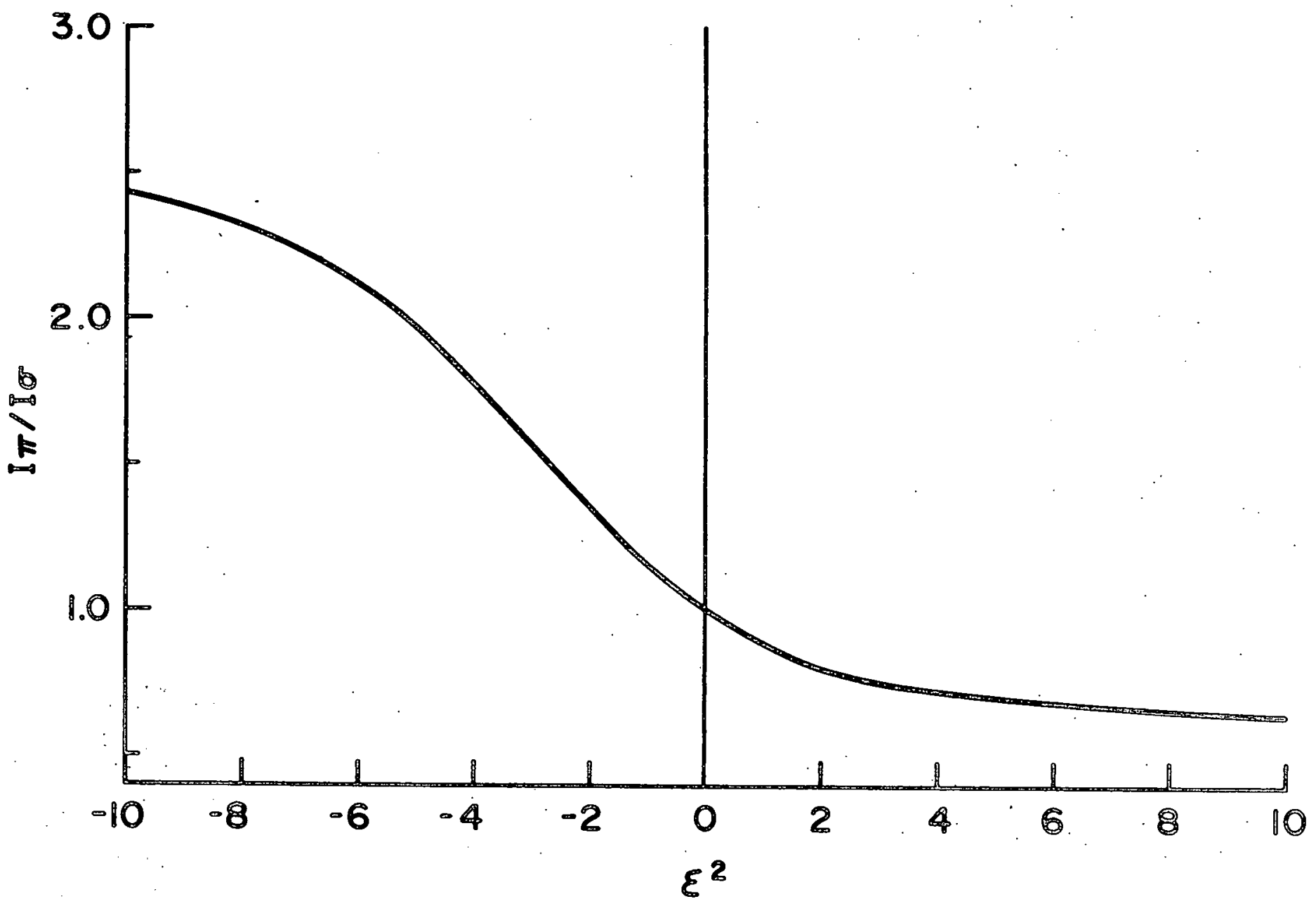

Figure 3. Calculated quadrupole doublet intęnsity ratio for an axially symmetric electric field gradient as a function of $\varepsilon^{2}=x^{2}\left[\left\langle z^{2}\right\rangle-\left\langle x^{2}\right\rangle\right]$. 
For $\varepsilon^{2}: 0$ we get

$$
\frac{I}{I_{\sigma}}=\frac{\left(6 \varepsilon^{2}+3\right) \sqrt{\pi} \operatorname{erf}(\varepsilon)-6 \varepsilon e^{-\epsilon^{2}}}{\left(10 \epsilon^{2}-3\right) \sqrt{\pi} \operatorname{erf}(\varepsilon)+6 \varepsilon e^{-\varepsilon^{2}}} .
$$

These calculations were performed with a computer using numerical integration methods and the results are shown in Fig. 3.

Kagan $^{34}$, concludes from symmetry considerations that lattice vibrational frequencies parallel and perpendicular to the axis of symmetry are independent.. If one then: assumes harmonic lattice forces for the crysta1, the mean square displacements in both directions and thus $\epsilon^{2}$ can be calculated in terms of a Mossbauer characteristic temperature for each branch from the relations $f_{z}=\exp \left(-x^{2}\left\langle z^{2}\right\rangle\right)$ and $f_{x}=\exp \left(-x^{2}\left\langle x^{2}\right\rangle\right)$.

\section{Crystal Field Theory}

The primary cause of an electric field gradient at the nucleus of an $\mathrm{Fe}^{2+}$ ion is the asymmetric charge distribution of the 3d-"valence" electron. A smaller contribution is made by the charge distribution of the neighboring ions, but it is on $1 y$ on the order of ' $1 \%$. of the valence contribution. This outer valence electron will polarize the ferric-1ike core of the ferrous ion such that the core will make a significant contribution to the electric field gradient. This effect, however, can be accounted for quite easily by use of the Sternheimer ${ }^{35}$ core polarization factors

Ingalls $s^{36}$ has developed a method for computing the electric field gradient tensor in ferrous compounds. It consists in first applying 
the crystal field and spin orbit perturbations, appropriate to the particular compound, to the ${ }^{5} \mathrm{D}$ free. ferrous ion states. A statistical average is then taken over the electric field gradient tensor components produced by each of the resulting electronic states.

This method will be applied to the case of a ferrous ion occupying a cobalt site in $\operatorname{CoCl}_{2}{ }^{\prime}$. The six neighboring chlorine ions surrounding a cobalt site in this compound are arranged in an octahedron with a slight distortion along the (111) axis of the octahedron. This axis is one of trigonal symmetry and coincides with the c-axis of the crystal. The crystal field potential at the cobalt site may be expanded in spherical harmonics, as follows:

$$
\mathrm{V}=\sum_{\ell, \mathrm{m}} \mathrm{A}_{\ell}^{\mathrm{m}} \mathrm{r}^{\ell} \mathrm{Y}_{\ell}^{\mathrm{m}}(\theta, \phi)
$$

This potential will have the same symmetry as that of the lattice, i.e. trigonal, and thus it has the explicit form

$$
V=A_{2}^{0} r^{2} Y_{2}^{0}(\theta, \phi)+A_{4}^{0} r^{4} Y_{4}^{0}(\theta, \phi)+A_{4}^{3} r^{4} Y_{4}^{3}(\theta, \phi)+A_{4}^{-3} r^{4} Y_{4}^{-3}(\theta, \phi)
$$

where an unimportant constant term and negligible higher order terms have been omitted. By changing to cartesian coordinates this potential can be written as the sum of a cubic and a trigonal term:

$$
\begin{aligned}
& \mathrm{V}=\mathrm{V}_{\text {cubic }}+\mathrm{V}_{\text {trigonal }} \text {, or } \\
& \mathrm{V}=\mathrm{C}_{4}\left(\mathrm{x}^{4}+\mathrm{y}^{4}+\mathrm{z}^{4}-3 / 5 \mathrm{r}^{4}\right)+\mathrm{B}_{2}^{0}\left(3 \mathrm{z}^{2}-\mathrm{r}^{2}\right) .
\end{aligned}
$$

Bleaney and Stevens ${ }^{37}$ have shown that the effect of the dominant 
cubic term is the splitting of the ${ }^{5} \mathrm{D}$ ferrous ion state into a $d_{\gamma}$ and a $d_{\epsilon}$ state with an energy separation of the order of $10^{4} \mathrm{~cm}^{-1}$. At ordinary temperatures $\left(\mathrm{kT} \sim 200 \mathrm{~cm}^{-1}\right)$. the upper $\mathrm{d}_{\gamma}$ state will not be populated and thus need not be considered in further calculations:- The lower $d_{\epsilon}$ state is three-fold degenerate, neglecting spin; an is represented by the wave functions,

$$
\begin{aligned}
& \frac{2}{3} \psi_{-2}+\cdots \frac{1}{3} \psi_{1} \\
& \because \because+\psi_{0}, \quad \text { and } \\
& \frac{1}{3} \psi_{-1}-\frac{2}{3} \psi_{-2} \text {, }
\end{aligned}
$$

where $\psi_{\mathrm{m}}=\mathrm{R}_{\mathrm{n} \ell}(\mathrm{r}) \mathrm{Y}_{\ell}^{\mathrm{m}}(\theta, \phi)$.

The problem of the solution of the radial part of the above wave functions, $R_{n \ell}(r)$, is avoided by replacing any integrals over the radial part by the expectation value of the operator involved, and relating these to free ion radial functions which can be evaluated.

The $d_{\varepsilon}$ level is further split in the case considered here by the trigonal field and the spin-orbit interaction. The Hamiltonian for the angular part of the wave functions expressed in terms of angular momentum operators is

$$
J C=-\lambda \vec{L} \cdot \vec{S}-\frac{\Delta}{3}\left(3 L_{z}^{2}-\vec{L}^{2}\right)
$$

where $\lambda$ is the spin orbit coupling constant and $\Delta$ is the trigonal field splitting. The operators $\vec{L}$ and $\vec{S}$ are the orbital angular momentum and spin operator's for the ${ }^{5} \mathrm{D}$ state. This perturbation problem can be 
simplified by noting that the $d_{\varepsilon}$ wavefunctions behave like a set of $|\vec{l}|=1$ wavefunctions if $-\vec{l}$ is substituted for $\vec{L}$. This introduction of the pseudoangular momentum ${ }^{38}$ enables us to represent the $d_{\epsilon}$ state by three wavefunctions $\varphi_{-1}, \varphi_{0}$, and $\varphi_{1}$ having a total angular momentum of 1 and $z$-components as indicated by the subscripts. The perturbation Hamiltonian can now be written as

$$
\mathcal{F}=\lambda \vec{\ell} \cdot \vec{s}-\frac{\Delta}{3}\left(3 \ell_{z}^{2}-\vec{\ell}^{2}\right),
$$

or with the introduction of the raising and lowering operators ${ }^{39}$,

$$
\mathcal{H C}=\lambda\left[\frac{l_{+} \mathrm{s}_{-}+\ell_{-}+s_{+}}{2}+l_{\mathrm{z}} s_{z}-\left(\frac{\Delta}{\lambda}\right) \frac{1}{3}\left(3 l_{z}{ }^{2}-\vec{l}^{2}\right)\right] .
$$

Since the interaction involves the electron spin, this Hamiltonian must be applied to the full $d_{\varepsilon}$ wavefunctions $\varphi_{i} x_{j}$, where $x_{j}$ is the spin part of the wavefunction with spin $|S|=2$. The solution of the problem. involves the diagonalization of the $15 \times 15$ perturbation matrix $(|\ell|=1,|S|=2)$ instead of the fu11 $25 \times 25$ matrix obtained without the introduction of the pseudoangular momentum.

This diagonalization can be accomplished numerically by using various values of $\Delta$ and $\lambda$ with a standard computer program which calculates both the eigenvalues and eigenvectors of the matrix.

Once the eigenvalues and eigenvectors have been found, the $\mathrm{V}_{\mathrm{zz}}$ component of the electric field gradient at temperature $\mathrm{T}$ can be found by taking a statistical average over the electronic states. . Thus,

$$
\overline{\left(V_{z z}\right)}=e q=\frac{\sum_{n}\left\langle V_{z z}\right\rangle_{n} e^{-\varepsilon_{n} / k T}}{\sum_{n} e^{-\varepsilon_{n} / k T}}
$$


with

$$
\left\langle\mathrm{V}_{\mathrm{zz}}\right\rangle_{\mathrm{n}}=\frac{4}{7} \mathrm{e}\left\langle\mathrm{r}^{-3}\right\rangle\left\langle\Phi_{\mathrm{n}} \mid 3 l_{\mathrm{z}}^{2}-{\overrightarrow{l^{2}}}^{2} \Phi_{\mathrm{n}}\right\rangle
$$

and

$$
\left|\Phi_{n}\right\rangle=\sum_{i j} a_{i j}\left|\varphi_{i} x_{j}\right\rangle
$$

where $\left|\Phi_{n}\right\rangle$ is the eigenvector of the $n$th eigenstate with energy $\varepsilon_{n}$. Since the crystal field, and thus the electric field gradient tensor, has axial symmetry on $1 \mathrm{y}_{\mathrm{zz}}$ will contribute to the quadrupole interaction.

It is convenient to write the electric field gradient component in terms of $(4 / .7) \mathrm{e}\left\langle\mathrm{r}^{-3}\right\rangle$ and a dimensionless statistical reduction factor $F(\Delta, \lambda, T)$. This reduction factor is shown; in $F$ ig. 4. as a function of $\mathrm{kT} / \lambda$. for several values of $\Delta / \lambda$. In this form $F(\Delta, \lambda ; \mathrm{T})$ is independent of the magnitude of $\Delta$ and $\lambda$ and applies to all compounds having an axially symmetric crystalline field for which $\Delta>0$.

The quadrupole splitting can now be written

$$
\Delta E=\frac{e^{2} \mathrm{qQ}}{2}=\frac{2}{7} \mathrm{e}^{2} \mathrm{Q}(1-\mathrm{R}) \alpha^{2}\left\langle\mathrm{r}_{0}{ }^{-3}\right\rangle \mathrm{F}\left(\Delta, \alpha^{2} \lambda_{0}, \mathrm{~T}\right)
$$

where $Q$ is the $z$-component of the quadrupole moment of the first excited state; $R$ is the Sternheimer core polarization factor; and $\alpha^{2}$ is the covalency factor, ${ }^{40}$ which accounts for the reduction in the values of $\lambda$ and $\left\langle r^{-3}\right\rangle$ from their free ion values $\lambda_{0}$ and $\left\langle r_{0}^{-3}\right\rangle$. Taking the Inga11's estimate of $Q=0.29 \mathrm{~b}$ and the common $1 \mathrm{y}$ used values of $\mathrm{R}=+0.32$ and $\left\langle x_{0}^{-3}\right\rangle=4.8$ a.u., and using for the free ferrous ion value of $\lambda_{0}$ the 




Figure 4. Quadrupole splitting reduction factor, $F(\Delta, \lambda, T)$, as a function of $\mathrm{kT} / \lambda$ with $\Delta / \lambda$ as a parameter for the case of an axially symmetric crystal field with $\Delta>0$. 
theoretical value $40103 \mathrm{~cm}^{-1}$, the expression for the quadrupole splitting becomes,

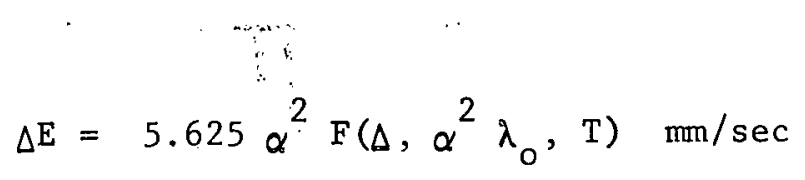

In this way the calculation of the quadrupole splitting:as a function of temperature has been reduced to the determination of a spectroscopic splitting factor $\Delta$ and a covalency factor $\alpha^{2}$. These two parameters can be found by fitting the calculated curves to the experimental data.

Ono, Ito and Fujita 19 have performed a similar crystalline field calculation in the isomorphous compound $\mathrm{FeCl}_{2} \cdot \AA$ discrepancy exists between their values for the quadrupole reduction factor and those calculated here. The difference increases with decreasing values of $\Delta / \lambda$. The origin of this discrepancy is not evident since Ono et.al. present their results on $1 y$ in graphical form. They obtain a value of 0.9 for the covalency factor, however, in $\mathrm{FeCl}_{2}$, which is outside the range of 0.67 to 0.8 reported by Ingalls for a wide variety of ferrous compounds. 


\section{EXPERIMENTAL TECHNIQUES}

\section{A. Sample Preparation}

Cobalt chloride samples were made by means of the following procedure. Radioactive $\mathrm{Co}^{57}$ was obtained from various suppliers in the form of an HCl solution. This $\mathrm{Co}^{57}$ solution was added to an aqueous solution of 20 to $30 \mathrm{mg}$ of reagent grade $\mathrm{CoCl}_{2}$ : The mixture was dried in air to the anhydrous salt in a quartz or porcelain crucible on a hot plate at $150^{\circ} \mathrm{C}$. The anhydrous powder was then sealed in an aluminum or copper cup on to which a Mylar or Lucite cap was cemented. This latter step was necessary because of the well known tendency of $\mathrm{CoCl}_{2}$ to form its hydrated compounds upon contact with atmospheric humidity.

The preparation of $\mathrm{CoF}_{2}$ samples required a more involved procedure. Although $\mathrm{CoF}_{2} \cdot 4 \mathrm{H}_{2} \mathrm{O}$ can be made quite easily by precipitation from an aqueous solution, the salt will hydrolyze when heated in an attempt to form the anhydrous salt. Consequently, the anhydrous samples were prepared by reacting anhydrous hydrogen fluoride gas with anhydrous cobalt chloride containing $\mathrm{Co}^{57}$ at $700^{\circ} \mathrm{C}$.

$$
\mathrm{CoCl}_{2}+2 \mathrm{HF} \rightarrow \mathrm{CoF}_{2}+2 \mathrm{HCl}
$$

The high chemical reactivity of $\mathrm{HF}$ necessitated the use of specially constructed apparatus. The $\mathrm{CoCl}_{2}$, which was prepared in the same mannex as that described above, was placed in a boat formed from 0.0025 " platinum foil. The reaction took place in a nickel furnace tube which had a 
copper screw cap. The cap itself had brass fittings which were used as inlet and outlet ports for the $H F$ gas. The furnace tube was aged by passing $\mathrm{HF}$ through the tube for several hours while it was in an $800^{\circ} \mathrm{C}$ furnace. In this way a thin coating of nickel fluoride was built up on wal1s of the tube. This coating inhibited further reaction of the gas with the tube while the samples were being prepared. Anhydrous hydrogen fluoride with a quoted minimum purity of $99.9 \%$ was obtained from lecture bottles supplied by the Matheson Company, Inc.. The gas was led into the system through polyethylene tubing and vented through a bubbler constructed from a polyethylene wash bottle containing vacuum pump oil. A diagram of the apparatus is shown in Fig. 5.

Sample preparation was carried out with the following procedure. After the boat containing the $\operatorname{CoC}_{2}$ was closed inside the furnace tube, the air was flushed from the system by starting the HF gas flow. The tube was then inserted in a hot furnace, and the HF flow was maintained at a steady rate until the tube had been at the reaction temperature of approximately $700^{\circ} \mathrm{C}$ for 30 minutes. The HF gas was then shut off and replaced by a flow of helium gas. The tube was then removed from the furnace and the helium stream was maintained until the tube had cooled to room temperature. The sample boat was then removed from the tube and the powder sample was placed in an aluminum sample holder, which is described in detail later in Section $\mathbf{C}$.

In order to avoid significant electronic absorption of the $14.4 \mathrm{keV}$ gamma rays in the sources, the size of both the chloride and fluoride samples were limited to between 20 and $40 \mathrm{mg}$. The specific activity was kept to less than 5 millicuries which gave count rates which were within the counting capabilities of the detection system. 


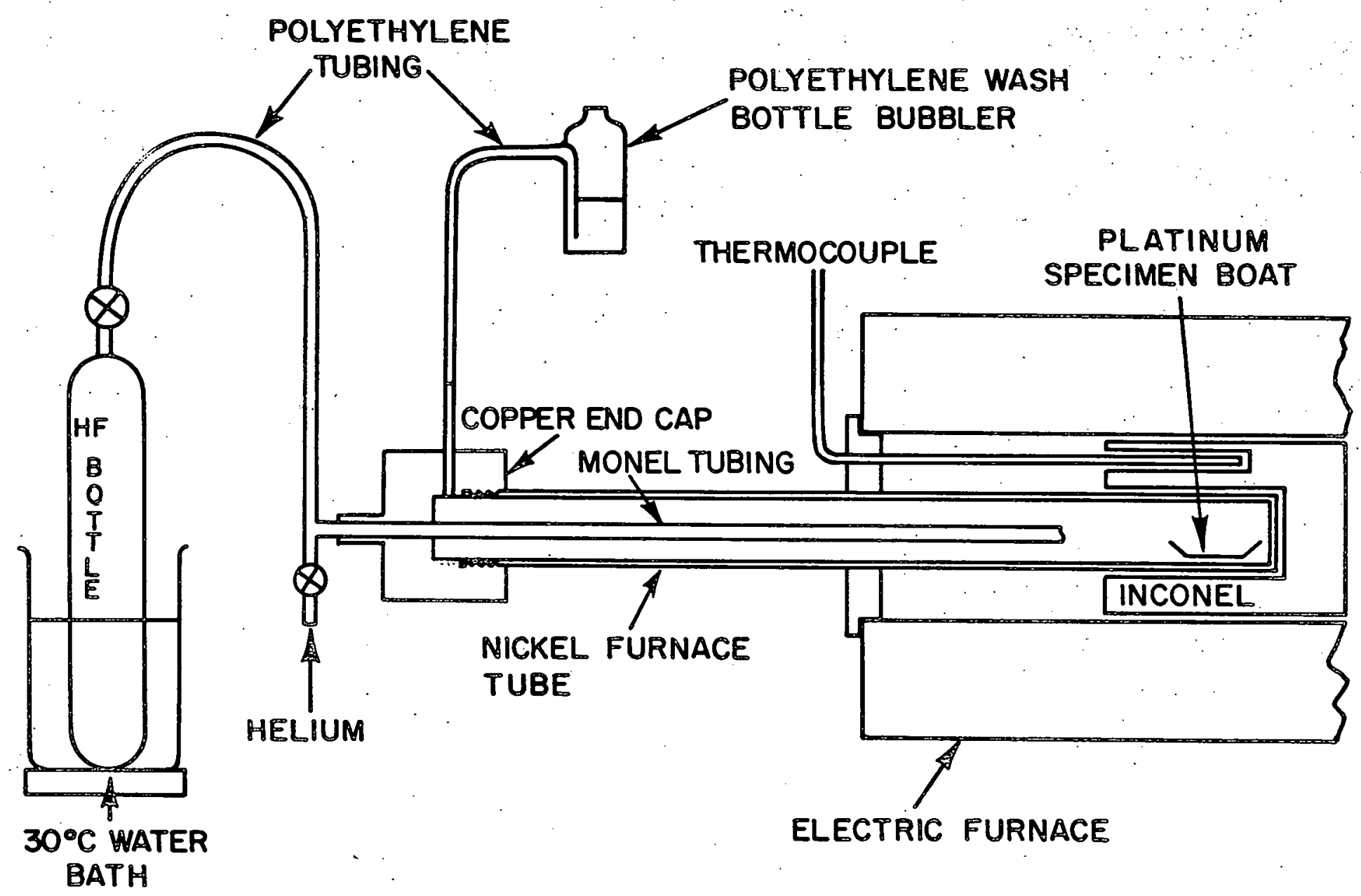

Figure 5. Schematic diagram of the apparatus used in the preparatian of $\mathrm{CoF}_{2}{ }^{\circ}$ 


\section{B. Mo̊ssbauer Spectrometèrs}

Two Mössbauer spectrometers were used in obtaining the velocity spectra of the sample specimens. The majority of the $\mathrm{CoCl}_{2}$ data was obtained with a reciprocating crank motion spectrometer 41 while most of the $\mathrm{CoF}_{2}$ :data was obtained with a constant velocity spectrometer. ${ }^{42}$ The former is a rapid scan device and, therefore, is not sensitive to changes in count rate due to radioactive decay or to drift in the counting electronics. The latter spectrometer has the advantage that data can be collected with high precision in regions of interest of the spectrum without collecting data at the same time in relatively unimportant regions. The actual choice of spectrometer for these measurements was based primaxily on availability and convenience.

The crank spectrometer system is shown schematically in Fig. 6 . The absorber is attached to a reciprocating shaft driven by a crank arm connected to the flywheel. Since the flywheel turns with a uniform rotational speed, $w$, the velocity of the absorber can be written as a function of the angle of rotation of the flywhee 1

$$
v(\theta)=-\omega\left[\cos \theta+\frac{a}{2 b} \cdot \sin 2 \theta\left(1-\frac{a^{2}}{b^{2}} \cos ^{2} \theta\right)^{-\frac{1}{2}}\right]
$$

where $\theta=\omega t, a$ is half the stroke distance of the crank and $b$ is the crank arm length (See Fig. 6).

The sine wave oscillator was set at a frequency approximately $2 \mathrm{x}$ $10^{4}$ times the rotational frequency of the flywheel. The sine wave output of the oscillator is converted by a Schmidt trigger into a uniform 


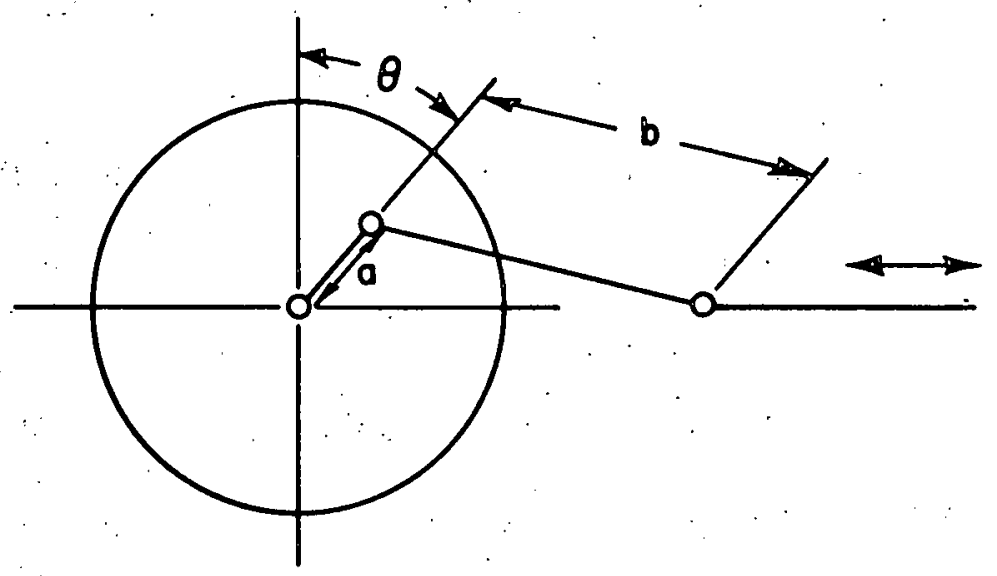

FLYWHEEL CRANK MOTION



Figure 6. Schematic diagram of the rapid scan Mössbauer crank spectrometer. 
pulse train which is fed into two decade scalers to produce approximately 200 evenly spaced pulses per flywheel revolution, which are used to advance the address of the multichannel analyzer. Thus, each channel of the analyzer represents a unique velocity interval, between $v$ and $v+\Delta v$, of the absorber motion. Sychronism between velocity and channel number is assured by using the photodiode output, which is triggered through a narrow slit on the flywheel, to reset the address of the analyzer to the first channel. Since this reset pulse also resets the two frequency step-down decades, sychronism is maintained to within 0.01 channels. The detector output pulses produced by the $14.4 \mathrm{keV}$ gamma are stored in the memory of the analyzer. Thus, the output of the analyzer is a record of the intensity of gamma ray transmission as a function of velocity.

In Fig: 7 the constant velocity spectrometer is shown schematically. The drive mechanism of this instrument is a Gaertner microscope slide on which either the source or absorber is mounted. The slide is driven at a constant speed, with alternatively positive and negative velocities, by a synchronous AC motor, which transmits power to the slide through a Metron variable ratio speed changer by means of pulleys and elastic belts.

Microswitches are used to reverse the direction of motion of the slide and control the operation of the data recording scalers. Five scalers are used to record the number of $14.4 \mathrm{keV}$ gamma rays detected while the slide is moving in a given direction, the time required to traverse the slide, and the total number of trips at a given speed. Since the distance of "travel of the slide in each direction is known, 


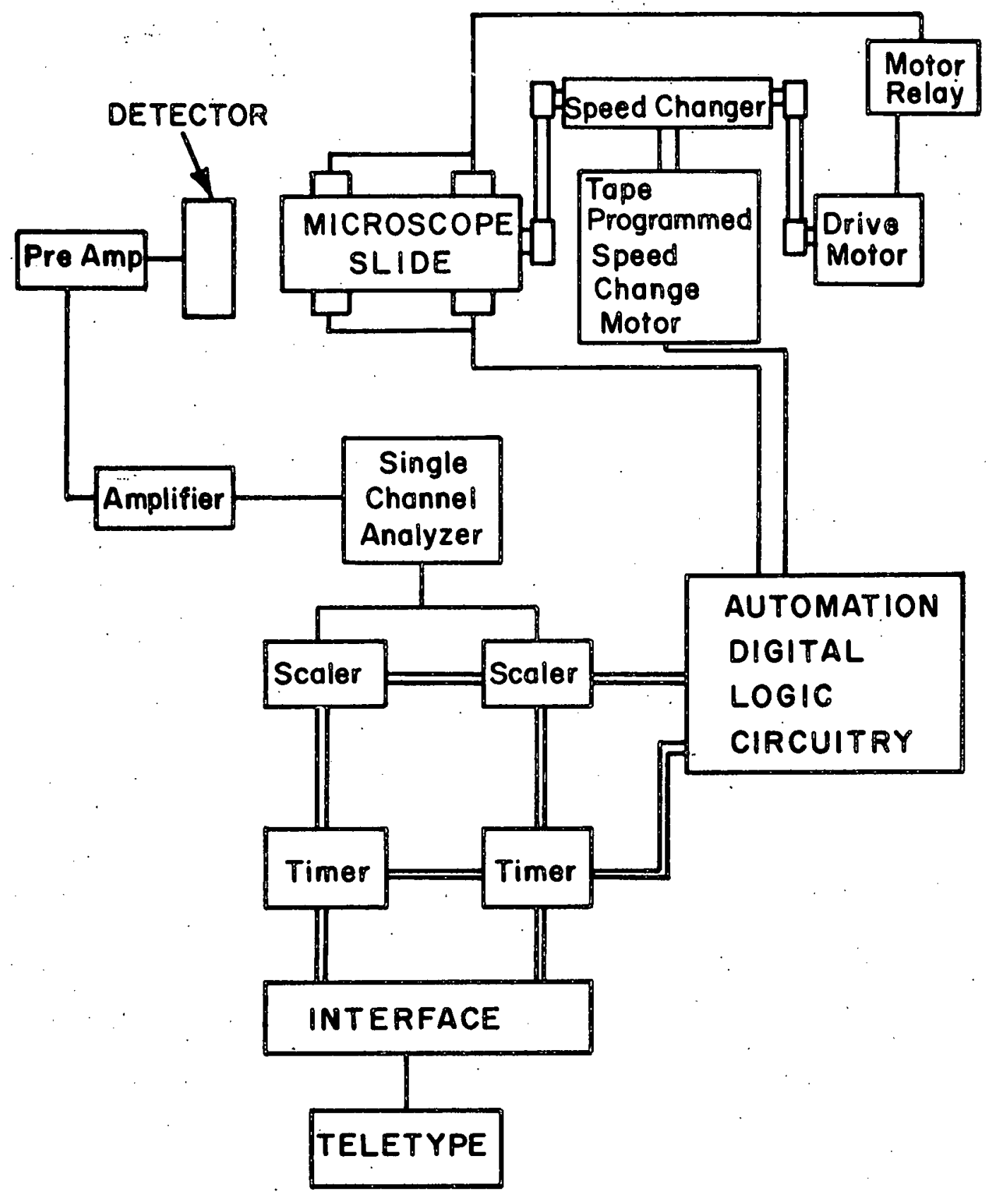

Figure 7. Schematic diagram of the constant velocity Mössbauer spectrometer. 
both count rate and velocity can be directly calculated from the data collected in the scalers.

In order to obtain a Mössbauer spectrum with this instrument, a paper tape is programmed by punching holes corresponding to the approximate relative velocities at.which data is desired. The spectrometer collects data for both positive and negative velocities until the desired statistical precision is obtained. The information contained in the scalers is then printed out and the speed changer.is automatically set for the next speed indicated on the prögram tape. The scalers are reset, and data accumulation resumes at this new speed. This process repeats itself automatically until the entire spectrum has been scanned.

\section{Temperature Measurement and Control}

Mössbauer measurements above $77^{\circ} \mathrm{K}$ were done in a stainless steel dewar with a single vacuum space between the liquid coolant and the external dewar walls. The source was mounted on a copper cold finger, which was aligned with a berylium window mounted on the outer wall of the dewar. The copper cold finger had a chamber accessible from the coolant space of the dewar into which a Watlow Firerod heating element could be inserted for the purpose of raising the temperature above $300^{\circ} \mathrm{K}$. Below $300^{\circ} \mathrm{K}$ the sample was maintained at a constant temperature by filling the dewar with coolants having either fixed boiling, melting, or sublimation temperatures.

$\therefore$ Temperature control above room temperature was achieved by using a West thermocouple controller, which regulated the power fed to the heating element. The actual. sample temperature was measured in ${ }^{\circ}$ this 
range with a chromel-alumel thermocouple. Between $300^{\circ} \mathrm{K}$ and $77^{\circ} \mathrm{K}$ sample temperatures were measured with a copper-constantan thermocouple. Below $77^{\circ} \mathrm{K}$ temperature stability was achieved by using an electronically controlled heater in conjunction with a liquid helium cold reservoir. Fig. 8 shows the arrangement employed. The cold finger was mounted on the cold working surface of a Texas Instrument CLF-1 Cryoflask, a lightweight liquid helium dewar, which uses the vaporized helium to cool the radiation shield instead of the usual liquid nitrogen jacket. Since the ratio of the thermal conductivities of aluminum and monel is approximately $10^{3}$ in the range of $4.2^{\circ} \mathrm{K}$ to $50^{\circ} \mathrm{K},{ }^{43}$ most of the temperature difference between the heater and cold finger appears across the monel tube, and the aluminum sample holder is nearly isothermal.

Temperature measurements in this range were made either with a carbon resistance thermometer or a GaAs junction diode thermometer, manufactured by the Phylatron Corp., Columbus, Ohio. Hot molded carbon resistors, such as those manufactured by the Allen-Bradley Corp., obey the following empirical temperature-resistance relationship.

$$
T=\frac{A \log R}{(\log R-B)^{2}}
$$

where $A$ and $B$ are experimentally determined constants. The resistance of the carbon thermometer was measured with an $\mathrm{AC}$ Wheatstone bridge using an oscilloscope as a detector. The $0.01 \mathrm{~V} / \mathrm{div}$ sensitivity of the detector made it possible to measure the resistance of the thermometer to within one ohm with a power dissapation of less than one milliwatt. Carbon resistor thermometers, however, require frequent recalibration 
and may change characteristics drastically if the resistor is subjected to mechanical stress.

The GaAs temperature sensor, on the other hand, is relatively stable and has a useful range of sensitivity from $3^{\circ} \mathrm{K}$ to $400^{\circ} \mathrm{K}$, whereas the carbon resistor can be used only up to about $50^{\circ} \mathrm{K}$. . With a constant current in the forward direction, the voltage drop across the junction of the GaAs diode sensor obeys the relationship

$$
\mathrm{V}=\mathrm{A}+\mathrm{BT}+\mathrm{CT}^{2}+\mathrm{DT} \mathrm{T}^{3}
$$

to within 1 part in $10^{4}$ in the range from $4.2^{\circ} \mathrm{K}$ to $27.3^{\circ} \mathrm{K}$. A constant current power supply operating at 100 ma was used to power the device. The voltage drop across the device was measured with a Leeds and Northrup K-2 potentiometer. The standard 4 wire measurement method was employed to eliminate errors due to lead resistance. With the 100 ma bias current employed, the power dissapated in the thermometer was of the order of 0.1 milliwatts. Fig. 8 also shows a sample holder which was designed for use at all temperatures in the range from $4.2^{\circ} \mathrm{K}$ to $700^{\circ} \mathrm{K}$. The $0.0005^{\prime \prime}$ aluminum foil acts as both a window for the $14.4 \mathrm{keV}$ gamma ray and a gasket which seals off the sample space from air and water vapor. The a11 aluminum construction eliminates sealing problems due to differential thermal expansion. A $200 \mathrm{ohm}$ heater made of 3 mil constantan wire is wound around the circumference of the holder for use below $77^{\circ} \mathrm{K}$. Thermometers can be mounted in holes drilled in the body of the holder.

The cryogenic electronic temperature controller was constructed as a modified version of a similar device designed by Linsay. ${ }^{44}$ A diagram of the controller is shown in Figs. $9^{\circ}$ and 10 . A carbon resistor is 


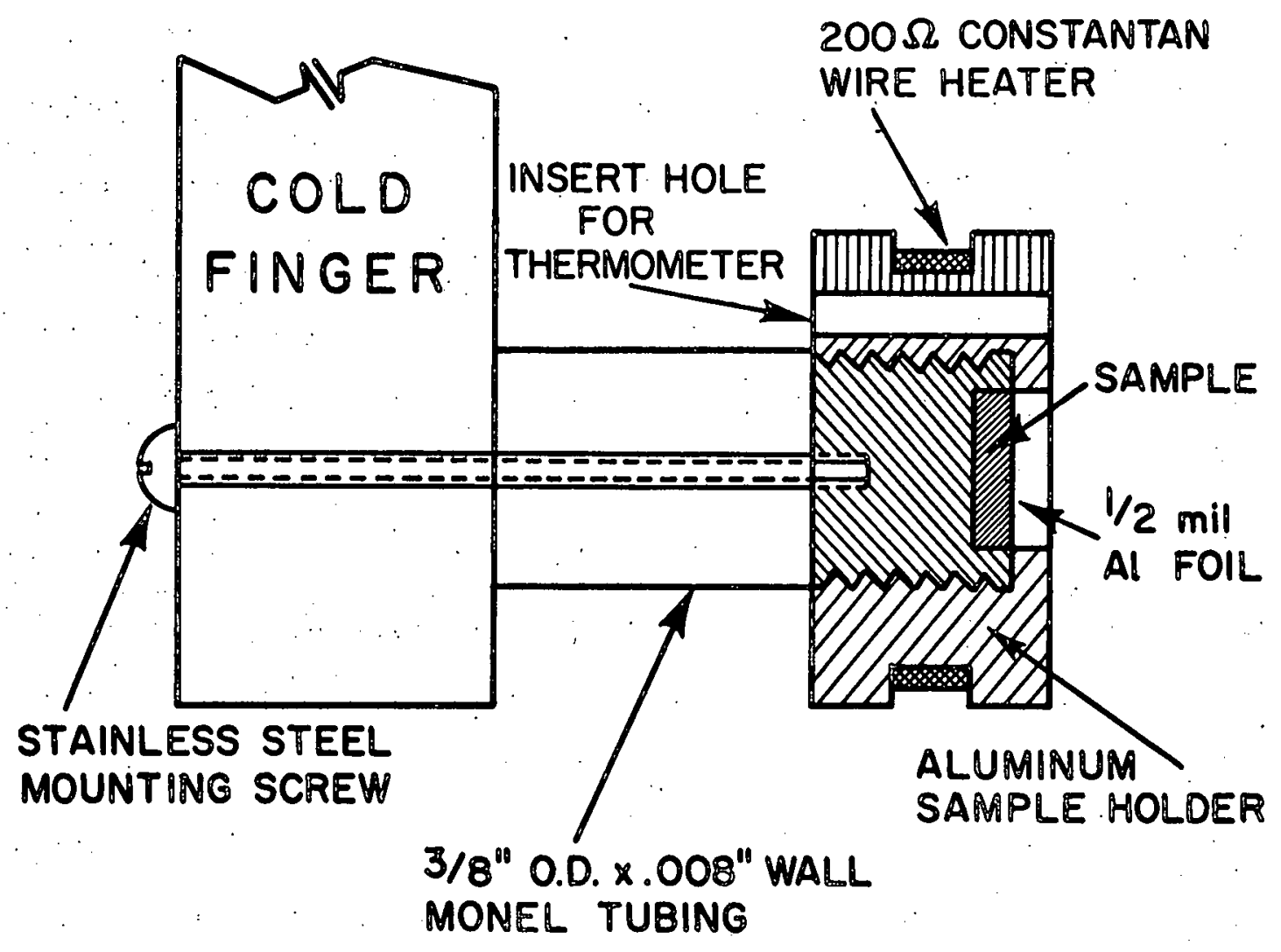

Figure 8. Radioactive sample holder and hellum dewar cold finger mounting. 
employed as the temperature sensing device because of its relatively large sensitivity in the operating range of the controller. Since the temperature measurements were made with a separate thermometer, precise calibration of the thermometer was not required.

This carbon resistor forms one arm of an AC Muller bridge. This type of resistance bridge is of the equal arm type in which the lead wires of the resistance to be measured become part of opposite arms of the bridge because of the placement of the detector terminals. If the two leads are identical, thermal changes in the resistance of the lead wires do not affect the balance conditions of the bridge.

If the value of the sensing resistor changes from that at the temperature set point, an error signal appears across the detector output of the bridge. The amplitude of this error signal is a function of the deviation of the sensor resistance from its equilibrium value. The error signal is also in phase with the bridge oscillator voltage for deviations below the equilibrium value and $180^{\circ}$ out of phase for deviations above the equilibrium value.

- The magnitude of the error signal is increased by a factor of 1000 in the amplifier section which consists of three identical stages.. Each stage is a complomentary bootstrap amplifier with heavy negative feedback. The feedback is adjusted such that each stage has a gain of 10 with no measurable change in either gain or phase shift for $20 \%$ changes in the power supply voltages with signal frequencies from $10^{2}$ to $10^{4} \mathrm{~Hz}$. The output of the amplifier is fed into the signal input of the phase sensitive detector (PSD). The reference input of the PSD is supplied by the same oscillator used to power the Muller bridge. The 




THERMOMETER TEMPERATURE CONTROLLER BLOCK DIAGRAM RESISTOR

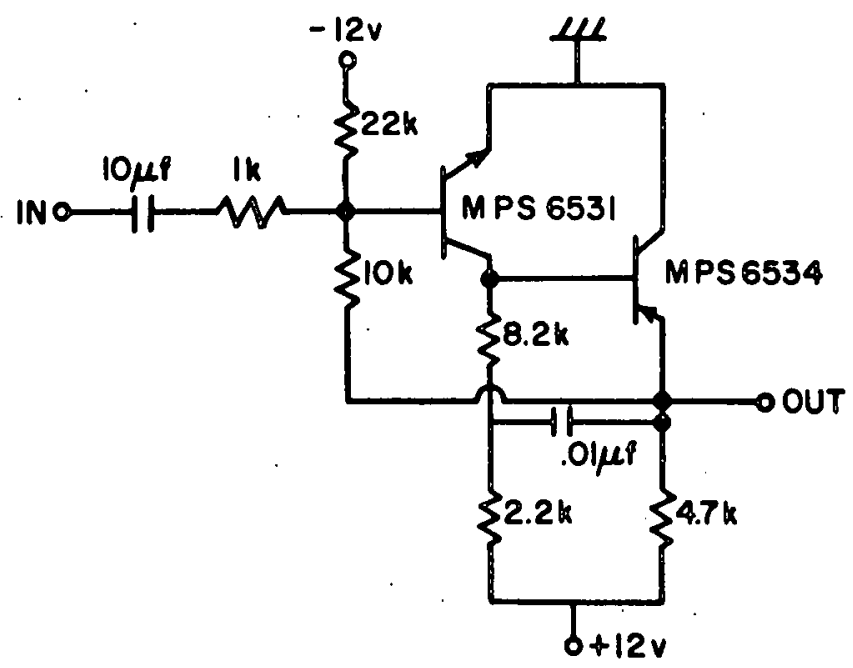

TYPICAL AMPLIFIER STAGE

Figure 9. Block diagram of the electronic temperature controller and schematic diagram of a typlcal amplifier stage. 


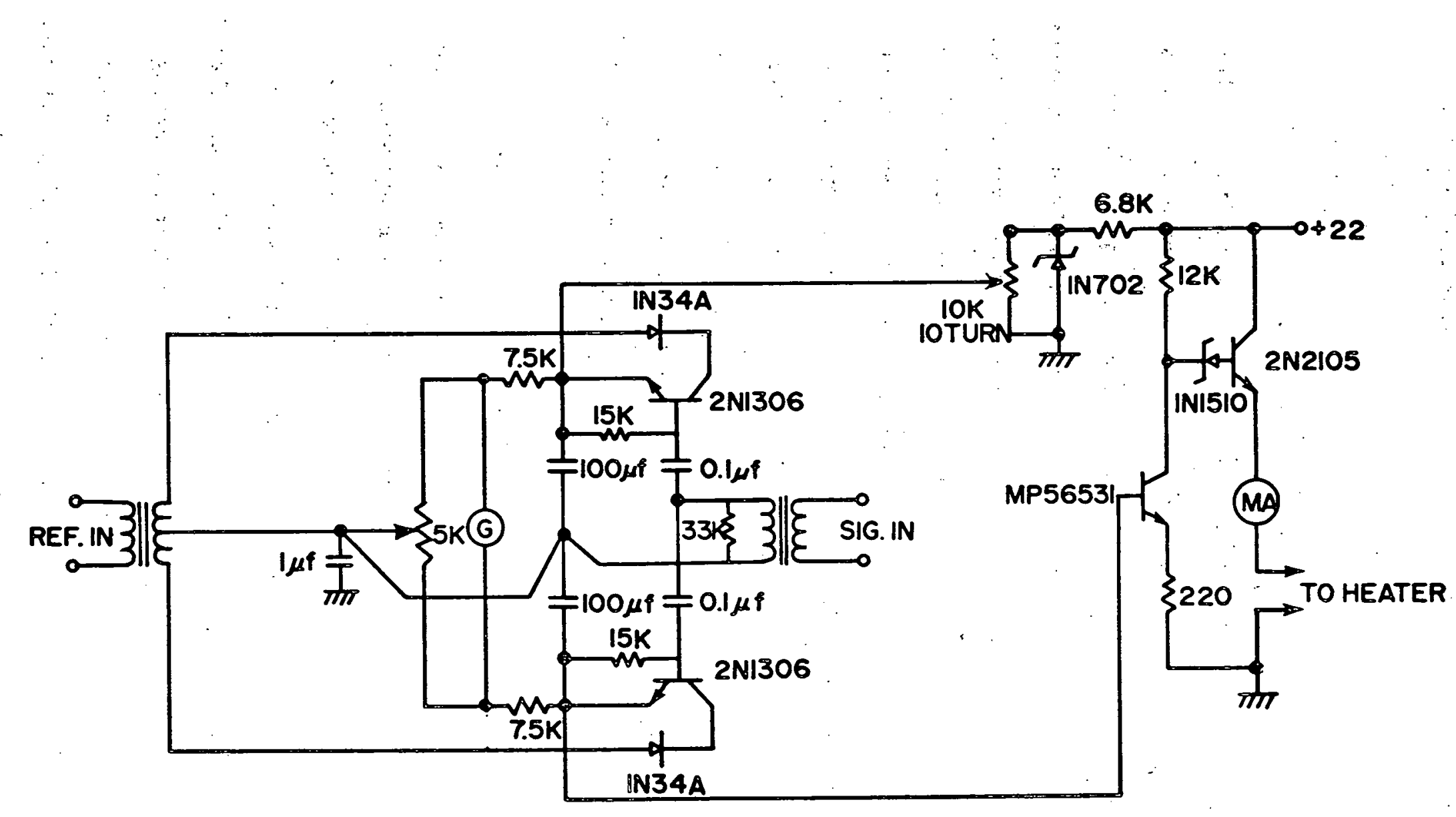

Figure 10. Detailed schematic diagram of the temperature controller phase sensitive detector. 
PSD first multiplies the signal and reference voltages and then integrates the product. The output is a: DC voltage of the form

$$
\mathrm{V}_{\mathrm{o}}=\mathrm{C} \mathrm{V}_{\mathrm{s}} \mathrm{v}_{\mathrm{r}} \cos \phi
$$

where $V_{S}$ and $V_{r}$ are the amplitudes of the signal and reference voltages, $\mathrm{C}$ is some constant dependent upon the circuit parameters and $\phi$ is the relative phase between the signal and reference voltages. Since only that part of the signal which is coherent with the reference voltage contributes to output, electrical noise and stray AC pickup are filtered out by this device. The output, then, of the PSD is a: DC voltage whose magnitude is a monotonic function of the deviation of the sensed temperature from the set point and whose polarity reflects deviations either above or below this set point. This voltage in turn is used to control a DC current amplifier which powers the sample heater. Thus an increase in sample temperature causes a decrease in power to the heater and, correspondingly, a decrease in sample temperature causes an increase in power to the heater.

The device is put into operation by adjusting the heater current until the measuring thermometer indicates the desired temperature. The Muller bridge is then balanced and the heater current is readjusted for its optimum value as indicated by a non-oscillating nul1 on the galvanometer which monitors the PSD output. Experience indicates that this device is capable of maintaining a temperature stability of better than $\pm I^{\circ} \mathrm{K}$ over a 12 hour period. Care must be given to the initial setting of the heater current, so that the controller does not have to correct for deviations of more than about $10^{\circ} \mathrm{K}$ from the nominal setting. Greater deviations than this cause an instability which makes the controller ineffective. 


\section{IV . . EXPERIMENTAL - RESULTS}

A. "Data: Reduction and Experimental Errors

The data output obtained from the velocity spectrometers were analyzed with a computer program which calculated the velocity and relative transmission for each datum point. The relevant experimental parameters of the spectra such as the peak positions, linewidths, and peak areas were obtained by a curve fitting analysis. Since the Breit-Wigner theory of radiation predicts that ideally the nuclear resonant absorption curves have a Lorentzian line shape, the experimental spectra are assumed to be described by an expression of the form

$$
A(v) \equiv \frac{I(\infty)-I(v)}{I(\infty)}=\frac{S \Gamma / 2 \pi}{\left(v-v_{0}\right)^{2}+\Gamma^{2} / 4}
$$

where $I(\infty)$ is the transmitted gamma ray intensity at Doppler velocities far removed from the resonant peak velocity, $v_{0} ; I(v)$. is the measured intensity as a function of velocity; $S$ is the area enclosed by the Lorentzian curve, $A(v)$ and $\Gamma$ is the full width at half maximum of the curve. Spectra which consist of more than one resonant absorption line are assumed to be described by a superposition of Lorentzian curves. The experimental spectra were fitted to a function of this form with a non-linear regression, least-squares computer program. This program is capable of fitting experimental data to an arbitrary function of 
one or more independent variables and several adjustable parameters. The parameter values are adjusted such that the sum of the squares of the residuals is a minimum, by using an algorithm due to D. W. Marquardt. 45 The computer program.used is an extensive revision of the original program (Share Distribution. No. 3094) made by A. J. Becker of Purdue. This revised version is simpler and more flexible than the original.

The error in the experimental data consists of the error in determining the velocity of each datum point and the statistical error in the determination of the gamma ray intensity. The error in the velocity determination is of the order of $0.1 \%$ and is small in comparison with other errors. Since radioactive decay processes obey Poisson statistics, the fractional standard error in the measured gamma ray intensity is $1 / \sqrt{N}$, where $\mathrm{N}$ is the total number of gamma rays counted at each datum point. In the experimental spectra presented here, $N$ was usually of the order $10^{5}$, which corresponds to a standard deviation of about $0: 3 \%$.

The errors associated with the parameters determined by means of the curve fitting program are a function of both the signal to noise ratio of the data and the deviation of the expeximental line shape from a Lorentzian curve. The signal to noise ratio can be defined as the ratio of the relative percentage absorption in the peak to the percentage statistical deviation. This ratio varies from about 50:1 for the quadrupole split patterns to as smál1 as 5:1 for some of the magnetic hyperfine patterns. The deviation of the experimental line shape from a true Lorentzian curve is caused by both random fluctuations in internal fields and finite absorber thickness. Only this last contribution is accessable to experimental control. The use of a thin absorber, 
however, decreases the percentage effect and, thus, a compromise must be made between line broadening and percentage effect. In most of the source experiments a $90 \%$ enriched $\mathrm{Fe}^{57}$ absorber was used, consisting of $\mathrm{Na}{ }_{2} \mathrm{Fe}(\mathrm{CN})_{6} \cdot 10 \mathrm{H}_{2} \mathrm{O}$ imbedded in a thin Lucite disk with an $\mathrm{Fe}^{57}$ concentration of $0.75 \mathrm{mg} / \mathrm{cm}^{2}$. This thickness gives a large percentage effect without significant line broadening. In some cases a $0.5 \mathrm{mg} / \mathrm{cm}^{2}$ or a $1.0 \mathrm{mg} / \mathrm{cm}^{2}$ absorber was used, either to increase the percentage effect at high temperatures or in the antiferromagnetic region where the percentage effect in the peaks is sma11, or because the $0.75 \mathrm{mg} / \mathrm{cm}^{2}$ absorber was in use in other experiments within the laboratory.

The curve fitting program calculates a standard error for each parameter using the usual statistical techniques for multiparameter analysis. These values are the ones quoted here. Other errors quoted represent estimates of standard error for quantities derived from the fitted parameters.

\section{B. Mo̊ssbauer Spectra Above the Né1 Temperature}

The Moossbauer velocity spectra of a $\mathrm{CoCl}_{2}$ source vs. a $\mathrm{Na}_{2} \mathrm{Fe}(\mathrm{CN})_{6}{ }^{\circ}$ $10 \mathrm{H}_{2} \mathrm{O}$ absorbex in the temperature range from $27^{\circ} \mathrm{K}$ to $670^{\circ} \mathrm{K}$ are shown in Fig. 11. The solid lines were calculated using the values of the parameters obtained from the curve fitting analysis with the assumption that each peak had the same linewidth. The results of the curve fitting are summarized in Table 2. These $\mathrm{C}_{\mathrm{C}} \mathrm{l}_{2}$ quadrupole split spectra show three important features. The first is the unusually small quadrupole splitting for a ferrous ion. Typical ferrous ion splittings range from $2 \mathrm{~mm} / \mathrm{sec}$ to $4 \mathrm{~mm} / \mathrm{sec}$ at room temperature ${ }^{36}$ in contrast with the present 


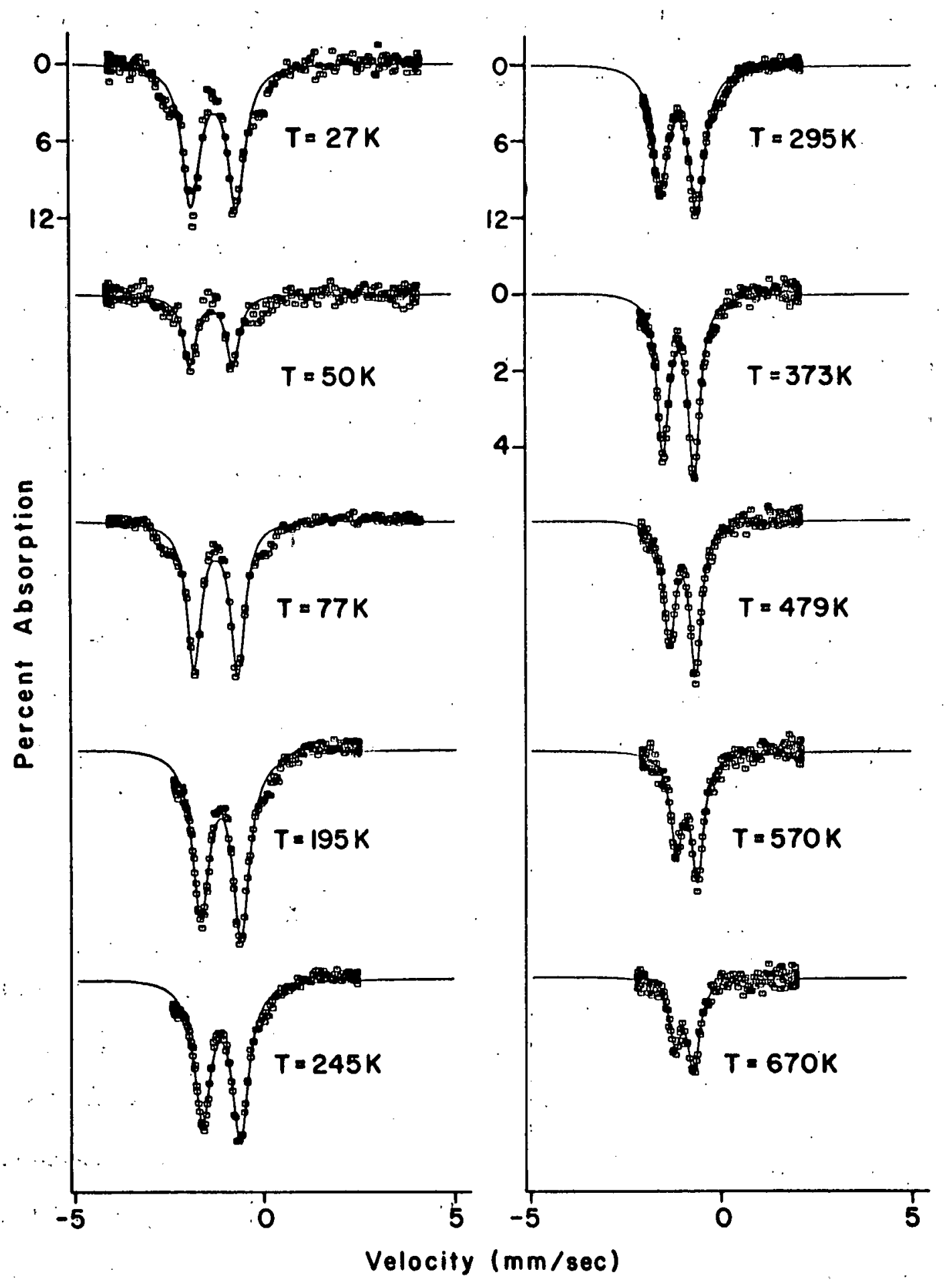

Figure 11. Mössbauer spectra of $\mathrm{CoCl}_{2}$ as a function of temperature above the Neel temperature. The same vertical scale is used for the data on the left and the larger scale applies to the data between $373^{\circ} \mathrm{K}$ and $670^{\circ} \mathrm{K}$. 
TABLE 2 。

Experimentally measured parameters of the Moissbauer spectra of $\mathrm{CoCl}_{2}$ above the Neel temperature.

\begin{tabular}{|c|c|c|c|c|c|c|c|}
\hline$\left({ }^{\mathrm{T}} \mathrm{K}\right)$ & $\mathrm{s}_{1}$ & $\mathrm{~S}_{2}$ & $\begin{array}{c}\mathrm{V}_{1} \\
\mathrm{~mm} / \mathrm{sec}\end{array}$ & $\begin{array}{c}\mathrm{V}_{2} \\
\mathrm{~mm} / \mathrm{sec}\end{array}$ & $\begin{array}{l}\mathrm{e}^{2} \mathrm{qQ} / 2 \\
\mathrm{~mm} / \mathrm{sec}\end{array}$ & $\begin{array}{r}\Gamma \\
\mathrm{mm} / \mathrm{sec}\end{array}$ & $\begin{array}{l}\left.\text { ab sorber } 2^{r}{ }^{57}\right) \\
\left(\mathrm{mg} / \mathrm{cm} \cdot \mathrm{Fe}^{2}\right)\end{array}$ \\
\hline 27 & 0.095 & 0.096 & -1.87 & -.68 & 1.19 & .56 & .75 \\
\hline 30 & 0.072 & 0.081 & -1.85 & -.66 & 1.19 & .53 & .75 \\
\hline 50 & 0.037 & 0.037 & -1.86 & $\therefore 72$ & 1.14 & .42 & .50 \\
\hline 77 & 0.080 & 0.085 & -1.84 &. .67 & 1.14 & .45 & .75 \\
\hline 197 & 0.108 & 0.122 & -1.74 & -.67 & 1.07 & .54 & 1.0 \\
\hline 245 & 0.089 & 0.103 & -1.67 & -.67 & 1.00 & .53 & 1.0 \\
\hline 295 & 0.036 & 0.043 & -1.59 & -.68 & 0.91 & .35 & .75 \\
\hline 373 & 0.024 & 0.028 & -1.48 & -.66 & 0.82 & .38 & .75 \\
\hline 479 & 0.018 & 0.023 & -1.34 & -.66 & 0.68 & .36 & .75 \\
\hline 570 & 0.014 & 0.020 & -1.20 & -.64 & 0.56 & .39 & .75 \\
\hline 673 & 0.009 & 0.014 & -1.10 & -.61 & 0.49 & .37 & .75 \\
\hline
\end{tabular}






Figure 12. Temperature dependence of the quadrupole splitting of $\mathrm{Fe}^{57}$ in $\mathrm{CoCl}_{2}$. The solid line was calculated with the use of crystal field theory. 
observed value of $0.91 \mathrm{~mm} / \mathrm{sec}$ for $\mathrm{CoCl}_{2}$ at $295^{\circ} \mathrm{K}$, which agrees with an earlier measurement at this temperature ${ }^{41}$. Secondly, the magnitude of the splitting is strongly temperature dependent, showing a $30 \%$ change.... in the temperature interval from $30^{\circ} \mathrm{K}$ to $300^{\circ} \mathrm{K}$. . Again, by contrast, most ferrous ion quadrupole splittings have less than a $10 \%$ variation over the same "temperature interva $1 .^{36}$ Both of these features can be explained in terms of the closely. spaced electronic energy' levels of the 3d-"valencẹ". electron of the $\mathrm{Fe}^{2+}$ ion. It is this electron which is the primary origin of the electric field gradient at the nucleus. In Fig. 12 the quadrupole splitting of $\mathrm{Fe}^{57}$ in $\mathrm{C} \odot \mathrm{C}_{2}$ is displayed as a function of temperature along with a theoretical curve obtained from crystal field calculations. This calculation is discussed in detail in Chapter $V$.

The third significant feature of these spectra is the asymetry in the relative intensity of the two lines of the doublet. The asymmetry increases monatonically with temperature and is interpreted as an example of the Goldanski-Karyagin effect. This effect, which arises from an anisotropy in the binding of the crystal, is expected in view of the layer like crystal structure of $\mathrm{CoC}_{i} 1_{2}$ :

Fig. 13 shows the experimental Mössbauer spectra of $\mathrm{CoF}_{2}$ and the fitted Lorentzian curves over the temperature interval from $42^{\circ} \mathrm{K}$ to $573^{\circ} \mathrm{K}$. All but the three highest temperature spectra were taken with a $0.75 \mathrm{mg} / \mathrm{cm}^{2}$ absorber. A $1.0 \mathrm{mg} / \mathrm{cm}^{2}$ absorber was used to increase the percentage effect at the higher temperatures. The spectra consist of two superimposed quadrupole doublets which are clearly resolved at the lower temperatures. The parameters which were obtained from the curve 


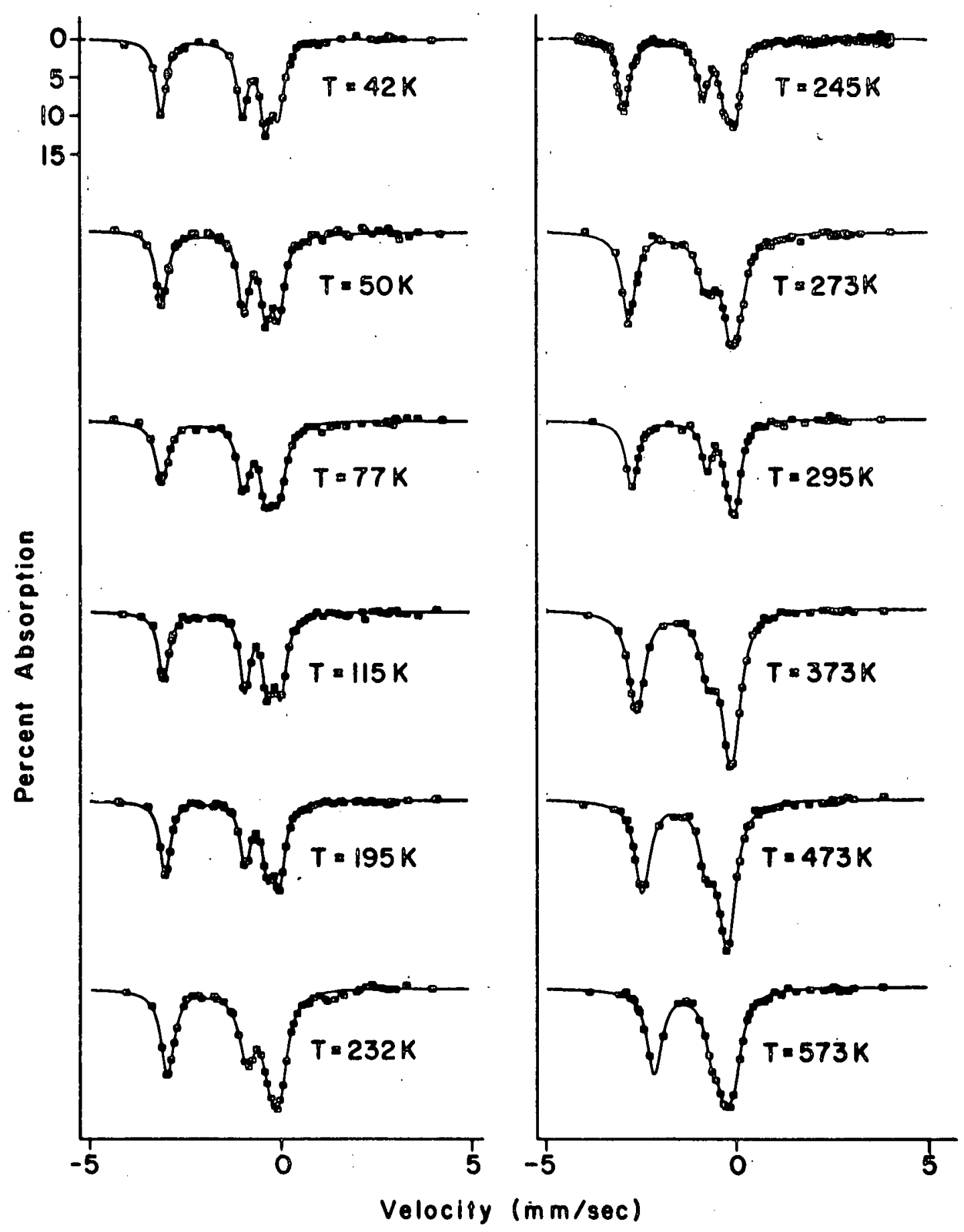

Figure 13. Mössbauer spectra of $\mathrm{CoF}_{2}$ as a function of temperature above the Neel temperature. The same vertical scale is used throughout. 
TABLE 3.

Experimentally measured parameters of the Mơssbauer spectra of $\mathrm{CoF}_{2}$ above the Neel temperature.

\begin{tabular}{|c|c|c|c|c|c|c|c|c|c|c|c|}
\hline $\mathrm{T}$ & $\mathrm{v}_{1}$ & $\mathrm{~V}_{2}$ & $Y_{3}$ & $v_{4}$ & $s\left(2^{+}\right)$ & $\mathrm{s}\left(3^{+}\right)$ & $\Gamma\left(2^{+}\right)$ & $\Gamma\left(3^{+}\right)$ & $\begin{array}{r}\mathrm{e}^{2} \mathrm{qQ} / 2 \\
\left(2^{+}\right)\end{array}$ & $\begin{array}{r}e^{2} q Q / 2 \\
\left(3^{+}\right)\end{array}$ & absorber \\
\hline$\left({ }^{\mathrm{O}} \mathrm{K}\right)$ & $\mathrm{mm} / \mathrm{sec}$ & $\mathrm{mm} / \mathrm{sec}$ & $\mathrm{mm} / \mathrm{sec}$ & $\mathrm{mm} / \mathrm{sec}$ &. & & $\mathrm{mm} / \mathrm{sec}$ & $\mathrm{mm} / \mathrm{sec}$ & $\mathrm{mm} / \mathrm{sec}$ & $\mathrm{mm} / \mathrm{sec}$ & $\left(\mathrm{mg} / \mathrm{cm}^{2} \mathrm{Fe}^{5.7}\right)$ \\
\hline 39 & -2.95 & -.88 & -.33 & -.02 & .050 & .050 & .47 & .45 & 2.93 & .55 & 1.0 \\
\hline 42 & -3.07 & -.93 & -.32 & .02 & .054 & .051 & .35 & .34 & 3.09 & .61 & 0.75 \\
\hline 50 & -3.07 & -.92 & -.33 & .01 & .055 & .053 & .42 & .38 & 3.08 & .59 & 0.75 \\
\hline 77 & -3.07 & -.92 & -.32 & .01 & .054 & .051 & .42 & .38 & 3.08 & .60 & 0.75 \\
\hline$\cdot 115$ & -3.06 & -.91 & -.32 & .03 & .049 & .049 & .34 & .31 & 3.09 & .59 & 0.75 \\
\hline 197 & -2.98 & -.87 & -.28 & .04 & .053 & .041 & .39 & .32 & 3.02 & .61 & 0.75 \\
\hline 232 & -2.94 & -.84 & -.22 & .04 & .053 & .047 & .42 & .50 & 2.98 & .62 & 0.75 \\
\hline 245 & -2.91 & -.84 & -.23 & .03 & .053 & .037 & .35 & .35 & 2.94 & .61 & 0.75 \\
\hline 273 & -2.85 & -.81 & -.16 & -.05 & .057 & .038 & .44 & .53 & 2.90 & .65 & 0.75 \\
\hline 295 & -2.76 & -.79 & -.24 & -.03 & .054 & .030 & .37 & .30 & 2.79 & .55 & 0.75 \\
\hline 373 & -2.63 & -.75 & -.22 & -.06 & .049 & .026 & .50 & .48 & 2.69 & .53 & 1.0 \\
\hline 473 & -2.39 & -.73 & -.39 & -.014 & .043 & .022 & .48 & .42 & 2.53 & .34 & 1.0 \\
\hline 573 & -2.16 & -.63 & -.31 & -.07 & .038 & .021 & .47 & .46 & 2.23 & .32 & 1.0 \\
\hline
\end{tabular}


fitting are summarized in Table 3 . It should be noted that for all temperatures the linewidth of the $\mathrm{Fe}^{3+}$. lines is less than or equal to that of $\mathrm{Fe}^{2+}$ lines within experimental error. This eliminates Auger aftereffects of the type proposed by Wertheim as a possible cause for this extra doublet, since the uncertainty principal requires that the $3+$ lines be wider than the $2+$ for this type of effect.

Three groups of experiments were performed in order to establish the possible origin of this $\mathrm{Fe}^{3+}$ doublet. Non-radioactive samples of cobaltous fluoride were prepared in the same manner as described previously for the radioactive Moossbauer samples. The chemical composition of these samples was tested gravimetrically by reducing them to the metal in hydrogen at $600^{\circ} \mathrm{C}$. Then assuming the sample had the composition CoF $_{x}$, the quantity $x$ was calculated from the weight of the original sample and the weight of the metal formed by the hydrogen reduction. Unfortunately, the experimental difficulties associated with the hydrogen fluoride preparation led to inconsistent results. The most consistent set of experiments, however, indicate that $\mathrm{x}$ lies between the limits of 1.9 and 2.1 when the $\mathrm{CoF}_{2}$ was prepared at $700^{\circ} \mathrm{C}$.

$\mathrm{X}$-ray powder diffraction patterns were made of both radioactive and non-radioactive samples of $\mathrm{CoF}_{2}$. These $x$-ray patterns were identical to those obtained with nominally high purity $\mathrm{CoF}_{2}$ obtained from commercial vendors, and they agreed with those found in reference tables. 46 These two experiments tend to rule out the possibility that non-stochiometry of the samples was responsible for the iron atom being found in both $3+$ and $2+$ charge states. A typical x-ray powder pattern of a nonradioactive sample prepared at $700^{\circ} \mathrm{C}$ is shown in Fig. 14. 


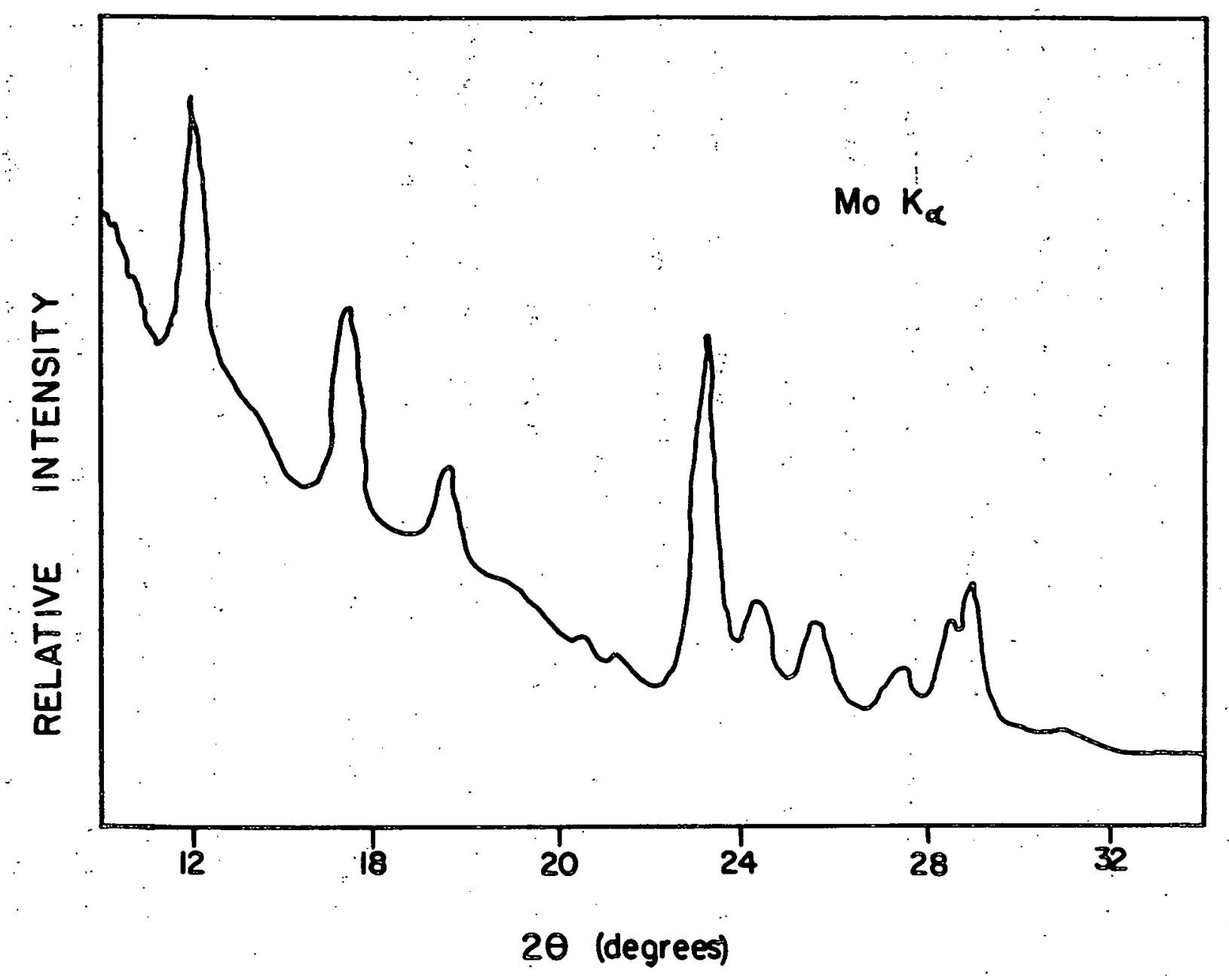

Figure 14. X-ray powder diffraction pattern of $\mathrm{CoF}_{2} \cdot$ The sample was prepared at $700 \mathrm{C}$, and Mo $\mathrm{K}_{\alpha}$ radiation was used in determining the powder 
In order to determine whether or not the origin of the extra $\mathrm{Fe}^{3+}$ spectrum was associated with the radioactive decay of $\mathrm{Co}^{57}$ a third type of experiment was performed. A sample of $\mathrm{CoF}_{2}$ was prepared with both $\mathrm{Co}^{57}$ and $0.5 \% \mathrm{Fe}^{57}$ by weight. The powder was sealed between two Lucite discs in such a way that it could be used either as a source or as an absorber. The Mössbauer spectra obtained from these combined source and absorber experiments are shown in Fig. 15. In the $\mathrm{CoF}_{2}$ absorber measurements a source consisting of $10 \mathrm{mc}$. of $\mathrm{Co}^{57}$ diffused into palladium watitm was used. The $0.75 \mathrm{mg} / \mathrm{cm}^{2}$ sodium ferrocyanide absorber was again used in the $\mathrm{CoF}_{2}$ source measurements. When the sample was used as a source, both the $\mathrm{Fe}^{2+}$, and $\mathrm{Fe}^{3+}$ quadrupole doublets again appear in the spectra. On the other hand, only the $\mathrm{Fe}^{2+}$ quadrupole doublet appears in the spectra obtained with the sample used as an absorber. Since iron tends to oxidize more "readily than cobalt to the $3+$ charge state, these combined source absorber experiments suggest that non-stochiometry is not responsible for $3+$ charge state of the $\mathrm{Fe}^{57}$ atoms. The origin of this effect is described in detail in Chapter VI.

\section{Mössbauer Spectra Below the Néel Temperature}

As the sample temperature was reduced below the Néel temperature of $25^{\circ} \mathrm{K}$, the $\mathrm{CoCl}_{2}$ Mössbauer spectra changed from a simple quadrupole split doublet to the complex pattern shown in Fig. 16. No further improvement in resolution was obtained beyond that shown in the figure at lower temperatures. The solid curve represents a fit to six superimposed Lorentzian curves. Attempts to fit the experimental spectra with 8 lines did not converge due to the lack of resolution. This difficulty 


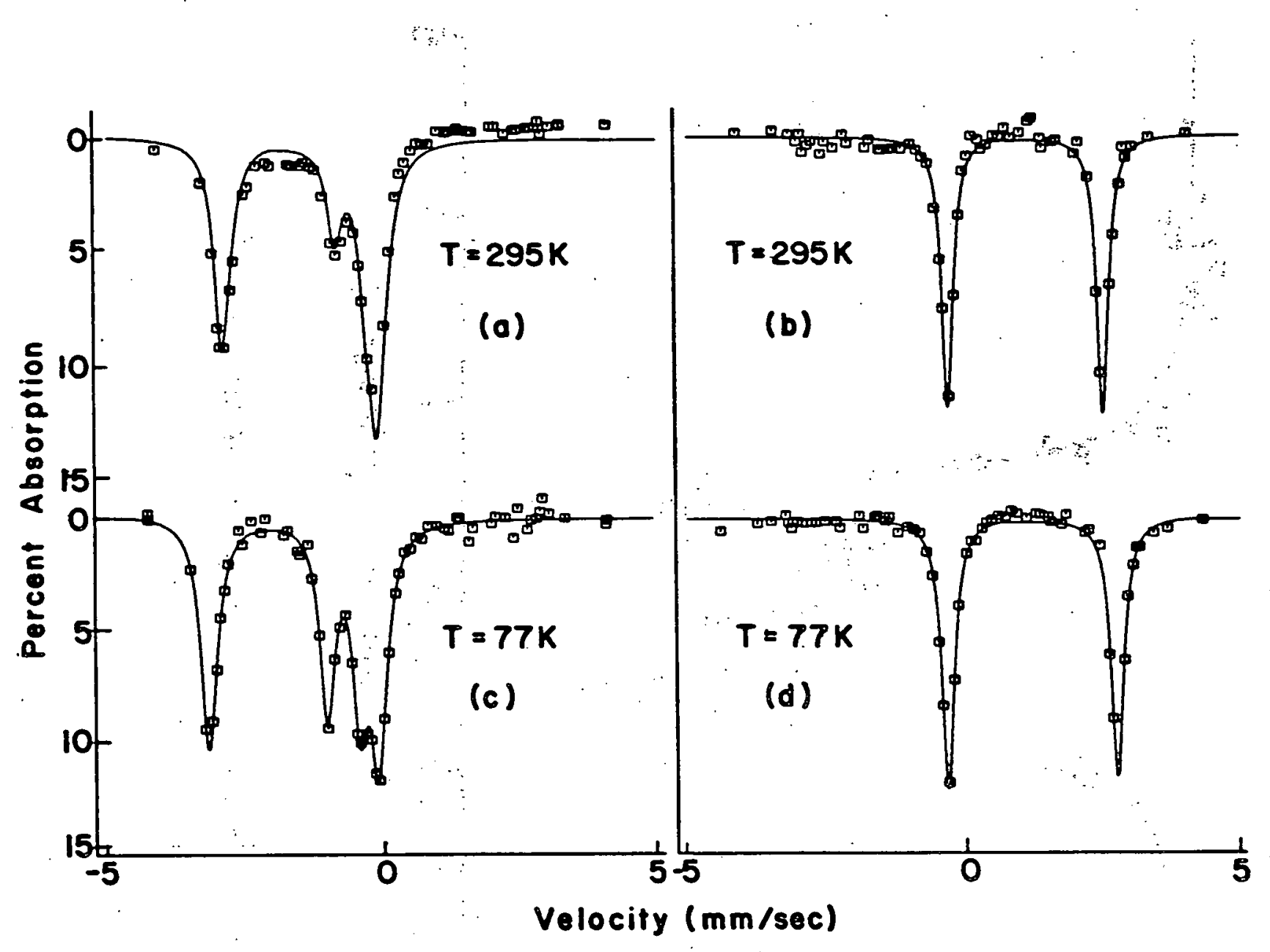

Figure 15. Mössbauer spectra of $\mathrm{CoF}_{2}$ doped with both $\mathrm{Co}^{57}$ and $\mathrm{Fe}^{.57}$. The data (a) and (c) resulted from using the sample as a source, and the data (b) and (d) resulted from using the sample as an absorber. 


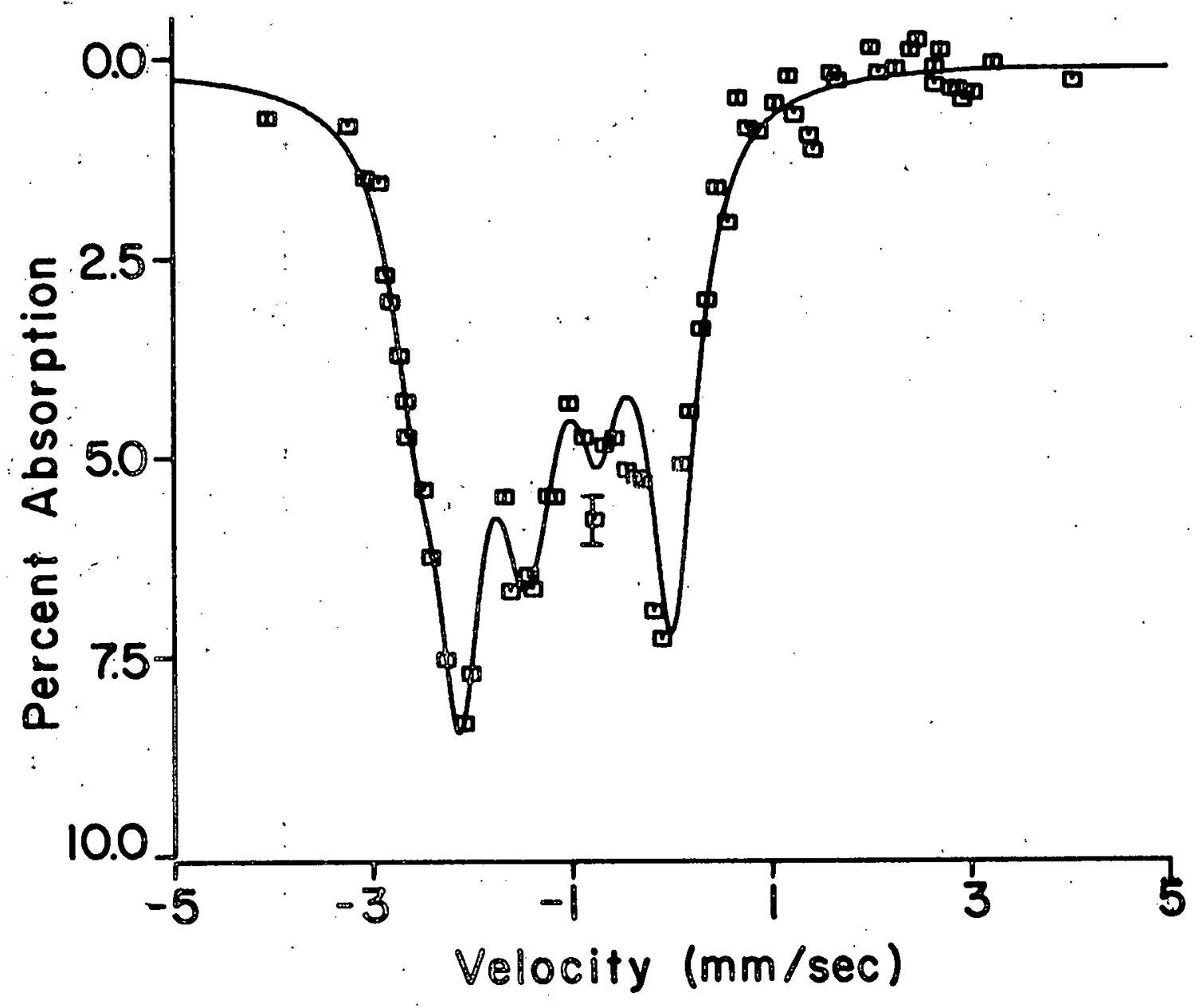

Figure 16. Hyperfine Mo̊ssbauer spectra of $\operatorname{CoCl}_{2}$ at $10^{\circ} \mathrm{K}$. 
was not crucial, and, in fact; the additional two lines which appear in the general case of a combined magnetic dipole and electric quadrupole interaction :frequently have very small intensities and are not often resolved.:

A: Mössbauer spectrum of $\operatorname{CoC}_{2}$ was also taken at $10^{\circ} \mathrm{K}$ with a magnetic field applied at right angles to the direction of emission of the gamma: rays....An extension tail-piece was made for the Cryoflask, which. could be inserted in the poles of a Pacific Electric Motor Co. electromagnet,; with a $4 \mathrm{~cm}$. gap." The maximum attainable field with this configuration of $18 \mathrm{kG}$ was used for the experiments. The absorber was attached to a 1 meter aluminum and stainless stee 1 boom. extending from the crankispectrometer into the inter-coil gap of the magnet in front of the gamma ray exit window of the tail piece. The proportional counter detector was mounted immediately behind the absorber.

The effect of this external field was to widen the pattern slightly, an: indication that the Mössbauer spectrum is magnetically split in the case of zero applied field, and that the complex pattern is not connected with some: relaxation phenomenon. No evidence was apparent of a sudden change in the magnetic hyperfine field with the application of the external field. "This: indicates that either an $18 \mathrm{kG}$ field is insufficient to cause such a transition or more probably the induced interlayer ferromagnetic interaction does not make a significant contribution to the field at the nucleus.

$\because$ This applied field measurement was also carried out with the sample at $77^{\circ} \mathrm{K}$ in order to determine the sign of the component of the electric field gradient $V_{z z}$. Although a field of this magnitude (18 $\mathrm{kG}$ ) is not 
sufficient to produce a resolvable splitting in the spectrum, it will broaden the $|3 / 2, \pm 3 / 2\rangle$ to $|1 / 2, \pm 1 / 2\rangle$ transition line more than the $|3 / 2, \pm 1 / 2\rangle$ to $|1 / 2, \pm 1 / 2\rangle$ 1ine, since the strength of the magnetic interaction is proportional to $\mathrm{m}$. In this case the greater broadening was observed for the line whose peak was located at $-1.8 \mathrm{~mm} / \mathrm{sec}$, which indicates that $\mathrm{q}$ is positive. This result is in agreement with both Mullen's ${ }^{41}$ studies of $\mathrm{CoCl}_{2}$ crystallites precipitated in an $\mathrm{NaCl}$ single. crystal with a partial orientation, and the results of the crystal field calculations for $\mathrm{Fe}^{2+}$ ions in $\mathrm{CoCl}_{2}$. This determination of the sign of $\mathrm{q}$ is necessary for the interpretation of the observed asymmetry in the $\mathrm{CoCl}_{2}$ Mössbauer spectrum taken above $\mathrm{T}_{\mathrm{N}}$.

Fig. 17 shows the antiferromagnetic Mössbauer spectra for $\mathrm{CoF}_{2}: \mathrm{Fe}^{57}$. The presence of both an $\mathrm{Fe}^{2+}$ and $\mathrm{Fe}^{3+}$ pattern complicates the spectra, which consist of at least 14 lines. The solid line represents a fit of the experimental data to a superposition of 12 lines consisting of a six line $\mathrm{Fe}^{3+}$ pattern and a six line $\mathrm{Fe}^{2+}$ pattern. It was necessary to make restrictive assumptions concerning the various parameters in order to obtain a fit to these data. The six lines in each of the two spectra were assumed to have the same linewidth. The $\mathrm{Fe}^{3+}$ pattern was further assumed to be a pure magnetically split case with no noticeable quadrupole interaction present. In this case the relative intensities and peak positions have known fixed ratios so that the number of parameters describing the Lorentzian curves can be reduced to 4; the linewidth, the relative intensity or area under one peak, the absolute position of one peak, and the total splitting of the $\mathrm{Fe}^{3+}$ pattern. This assumption is justified by the fact that magnetic interaction with the excited state 


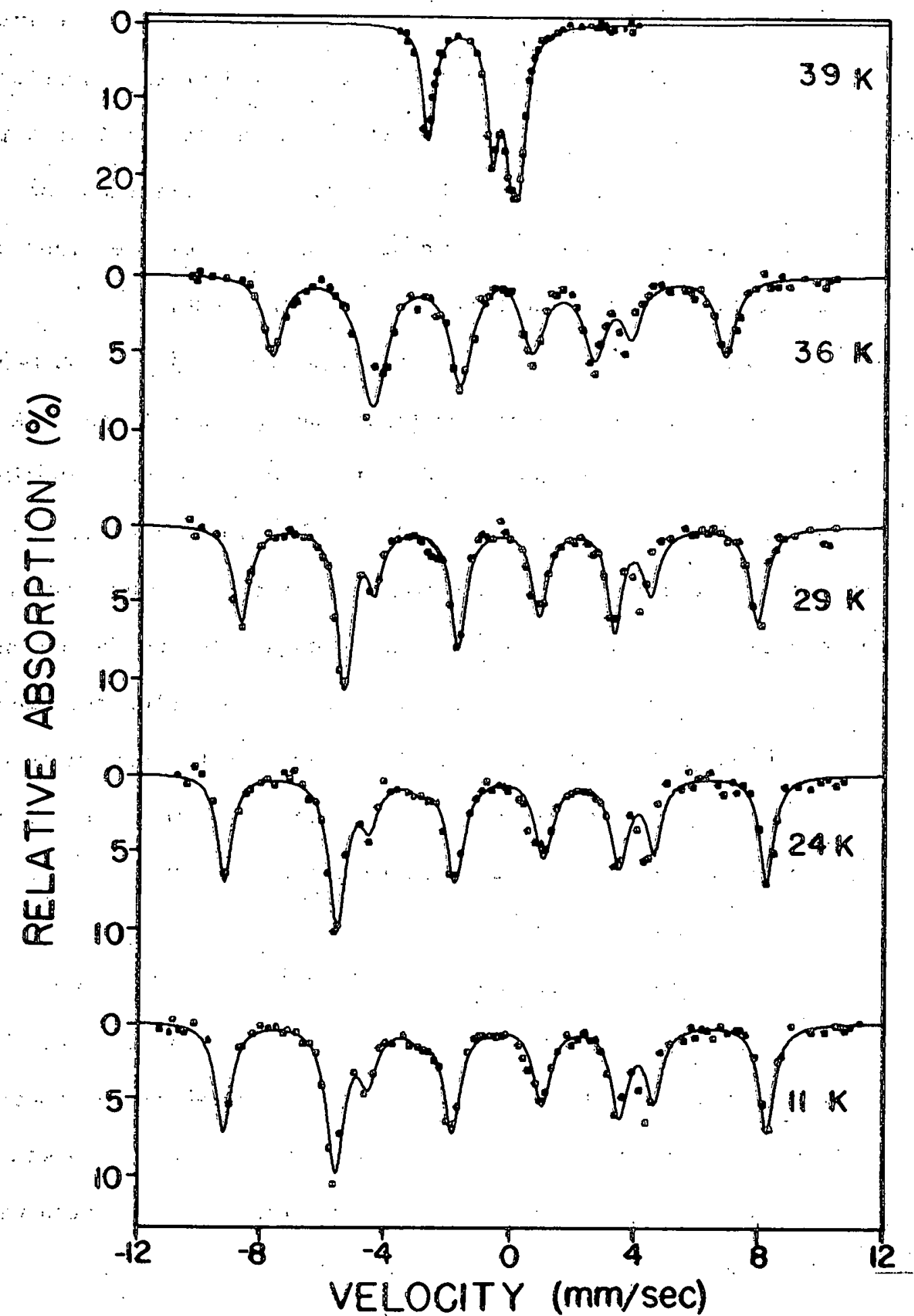

Figure 17. Hyperfine Mössbawer spectra of $\mathrm{CoF}_{2}$ as a function of temperature in the anti.ferromagnet ic region. 
in this case is about a factor of 30 greater than the quadrupole interaction observed for the $\mathrm{Fe}^{3+}$ ions just above the magnetic transition temperature. In the case of the $\mathrm{Fe}^{2+}$ pattern where the relative strength of the quadrupole interaction is much stronger, no restrictions were placed on the relative intensities and peak positions of the lines other than requiring the six lines to have the same linewidth.

It is evident from Fig. 17 that the transition from the non-magnetic spectrum to the antiferromagnetic spectrum takes place between $39^{\circ} \mathrm{K}$ and $36^{\circ} \mathrm{K}$. Since at $\mathrm{v}=0$ the transmission just above $\mathrm{T}_{\mathrm{N}}$ is near its minimum and is close to its maximum just:below $\mathrm{T}_{\mathrm{N}}$ at the same velocity, the magnetic transition temperature was determined by measuring the transmission of $14.4 \mathrm{keV}$ gamma rays from a $\mathrm{CoF}_{2}$ source through a sodium ferrocyanide absorber as a function of temperature at zero Doppler velocity. The results are shown in Fig. 18 and indicate a magnetic transition temperature of $38 \pm 1^{\circ} \mathrm{K}$. This value is lower than the neutron diffraction value $^{25}$ of $50^{\circ} \mathrm{K}$ but agrees with the specific heat measurement ${ }^{26}$ of $37.7^{\circ} \mathrm{K}$, which was discussed in Chapter $\mathrm{I}$.

It is interesting to note that both the $\mathrm{Fe}^{3+}$ and $\mathrm{Fe}^{2+}$ magnetic hyperfine patterns appear simultaneously at the same temperature. This is further evidence that the $\mathrm{Fe}^{3+}$ spectra is not caused by the source being a simple mixture of $\mathrm{CoF}_{2}$ and $\mathrm{CoF}_{3}$. For in this case, one would expect to observe two magnetic transition temperatures associated with each of the two compounds.

A CoF 2 absorber containing $0.5 \% \mathrm{Fe}^{57}$ by weight was prepared and mounted in the same manner as the combined source and absorber. This absorber showed only the $\mathrm{Fe}^{2+}$. spectra as was observed in the absorber 




Figure 18. Neel temperature determination for $\mathrm{CoF}_{2}$ by measuring the transmission of $14.4 \mathrm{keV}^{2}$ camma rays as a function of temperature at zero relative velocity. 
measurements with the combined source-absorber. A Mosssbauer spectra obtained from this absorber at $11^{\circ} \mathrm{K}$ with a cobalt in paladium source is shown in Fig. 19. The solid line is obtained by fitting the data to six Lorentzians. These six lines agree within experimental error with those of the $\mathrm{Fe}^{2+}$ portion of $11^{\circ} \mathrm{K} \mathrm{CoF}_{2}$ source spectra in both relative intensity and peak position, after a correction is made for both the velocity inversion of the absorber spectrum with respect to the source spectrum and the relative isomer shift of $\mathrm{Fe}$ in $\mathrm{Pd}$ with respect to $\mathrm{Na}{ }_{2} \mathrm{Fe}$ $(\mathrm{CN})_{6}: 10 \mathrm{H}_{2} \mathrm{O}$. The reason for determining the hyperfine constants from the $\mathrm{CoF}_{2}: \mathrm{Co}^{57}$ source instead of the $\mathrm{CoF}_{2}: \mathrm{Fe}^{57}$ absorber was the superior quality of the source data. This was because the absorber experiment had poorer count rates, which offset its advantage of having only an $\mathrm{Fe}^{2+}$ component in its spectra. 


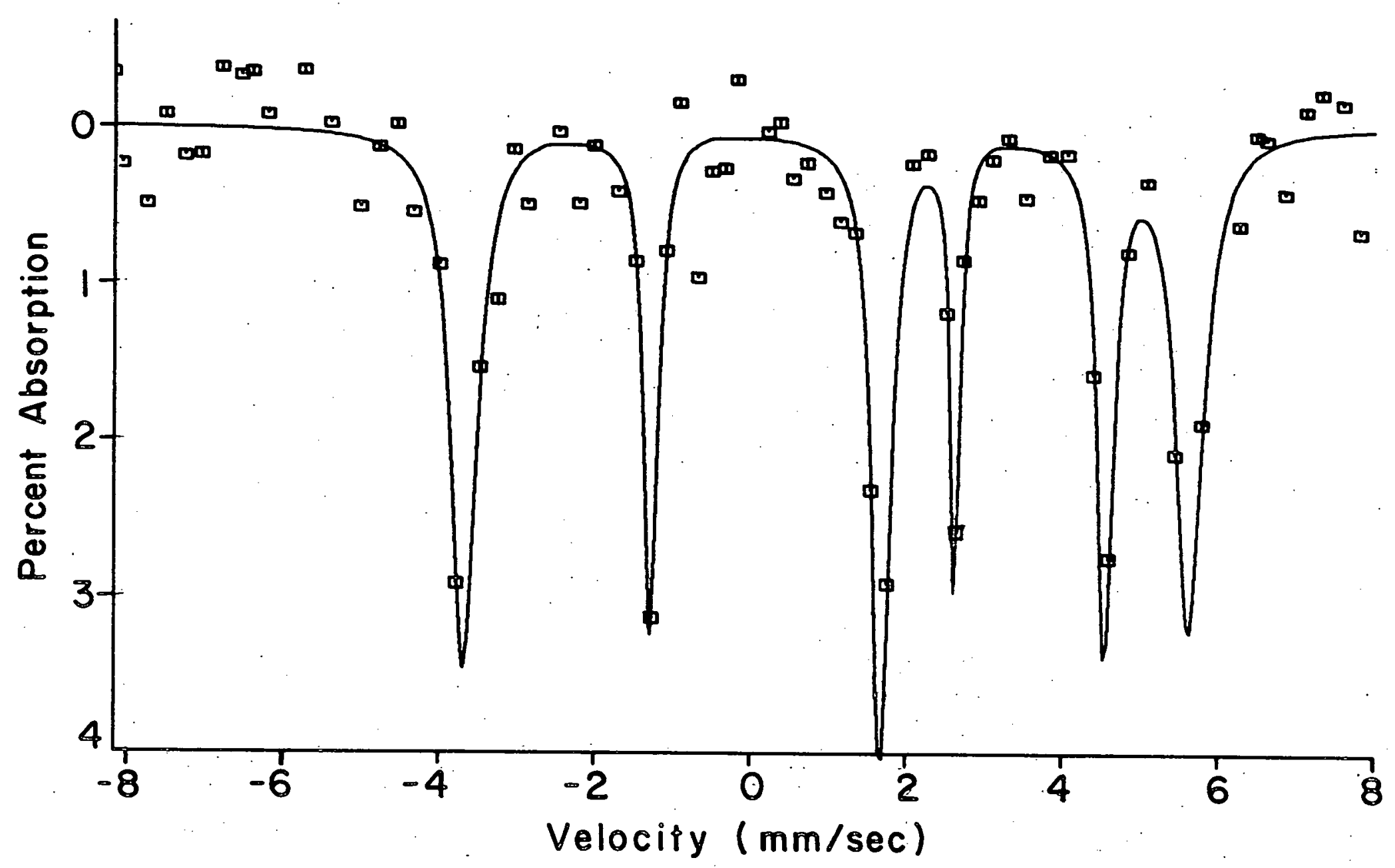

Figure 19. Hyperfine Mössbauer absorption spectrum of $\mathrm{CoF}_{2}$ doped with $\mathrm{Fe}^{57}$ at $11^{\circ} \mathrm{K}$ using a Co in
Pd source. 


\section{DISCUSSION OF EXPERIMENTAL RESULTS}

\section{A. $\mathrm{CoCl}_{2}$ Spectra Above the Néel Temperature $\therefore \therefore$}

Ingall's method of calculating the nuclear electric quadrupole splitting of a ferrous ion, as outlined in Chapter II, was used to obtain a theoretical fit to the measured values in the temperature range from $30^{\circ} \mathrm{K}$ to $670^{\circ} \mathrm{K}$. Using a least squares criterion, the optimum fit was obtained with a covalency factor $\alpha^{2}$ of $0.7_{3} \pm 0.1$ and a value of 2.5 \pm 0.25 for the parameter: $\Delta / \lambda$. The latter result gives a value of $\Delta=$ $190 \pm 25 \mathrm{~cm}^{-1}$ for the trigonal field splitting. The eigenenergies and eigenstates for this case are listed in. Table 4. The combination of the trigonal crystal field and the spin orbit interaction does not completely remove the degeneracy of the $d_{\varepsilon}$ state. As the table indicates this level is split into 6 two-fold degenerate sublevels and 3 non-degenerate subleve1s. The strong admixture of $\ell_{z}=0$ and $\ell_{z}= \pm 1$ angular momentum states in many of the eigenvectors explains the sma11 ferrous ion quadrupole splitting observed in this compound. These angular momentum states produce an electric field at the nucleus of $-4 / 7 \mathrm{e}\left\langle\mathrm{r}^{-3}\right\rangle$ and $+2 / 7 \mathrm{e}\left\langle\mathrm{r}^{-3}\right\rangle$, respectively. Thus, with the indicated admixture of states the lowest level could produce a quadrupole splitting of only $1.2 \mathrm{~mm} / \mathrm{sec}$ at $0^{\circ} \mathrm{K}$ when it alone is populated. The strong temperature dependence of the quadrupole splitting is easily explained by the relatively smali $50^{\circ} \mathrm{K}$ spacing between the two lowest levels. 
TABLE 4.

Eigenenergies and eigenstates of the $\mathrm{Fe}^{2+} \cdot \mathrm{d}_{\epsilon}$ level due to a trigonal field and spin-orbit interaction with $\Delta / \lambda=2.5$.




This analysis of the Mössbauer spectrum of $\mathrm{Fe}^{57}$ in $\mathrm{CoCl}_{2}$ was accomplished with methods originally devised for $\mathrm{Fe}^{57}$ in iron compounds. The high degree of quantitative agreement between theory and experiment in the analysis of the temperature dependence of the quadrupole splitting above $\mathrm{T}_{\mathrm{N}}$ is similar to that obtained by Ono et. al. ${ }^{19}$ in their work with isomorphous $\mathrm{FeCl}_{2}$.

\section{B. Goldanski-Karyagin Effect in $\mathrm{CoCl}_{2}$}

With the determination of the sign of $\mathrm{q}, \mathrm{v}_{\mathrm{zz}} / \mathrm{e}$, from the previous calculations and its agreement with the results of the applied magnetic field experiment, an identification can be made of the $|3 / 2, \pm 3 / 2\rangle$ to $|1 / 2, \pm 1 / 2\rangle, I_{\pi}$ and $|3 / 2, \pm 1 / 2\rangle$ to $|1 / 2 \pm 1 / 2\rangle, I_{\sigma}$, transitions, and the ratio $I_{\pi} / I_{\sigma}$. can then be calculated as a function of temperature from the data. The intensity ratios can be, in turn, compared with results of the calculations done in Chapter II using Karyagin's theory of quadrupole doublet asymmetry for an axially symmetric electric field gradient. In this way the parameter $\varepsilon^{2}=x^{2}\left[\left\langle z^{2}\right\rangle-\left\langle x^{2}\right\rangle\right]$, as a function of temperature, was obtained. Using the results of Chapter II, we are then able to find effective Mössbauer temperatures associated with the vibrations parallel to the symmetry axis, $z$, and in the direction, $x$, perpendicular to this axis.

The results are shown in Fig. 20 with the experimentally measured asymmetry shown in Fig. 20a. Fig. 20b is a plot of the corresponding asymmetry parameter as a function of temperature, with the solid line obtained from values of $\theta_{z}=140^{\circ} \mathrm{K}$ and $\theta_{\mathrm{x}}=300^{\circ} \mathrm{K}$ for the Mössbauer characteristic temperatures. At a lattice temperature of $300^{\circ} \mathrm{K}$ the 

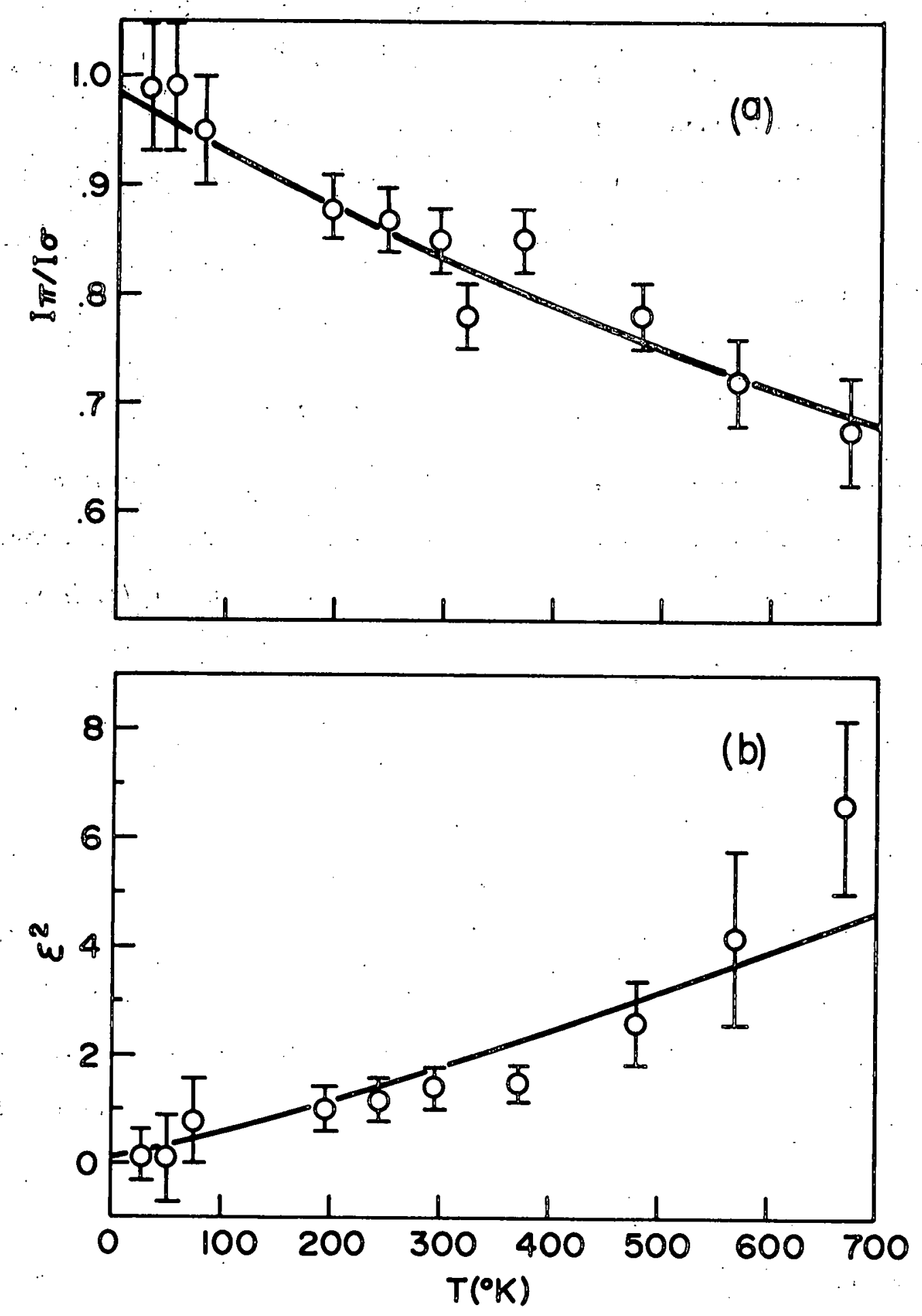

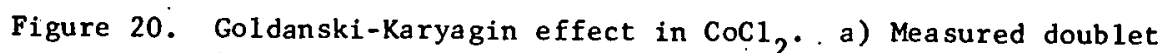
intensity ratio as a function of Eemperature, b) a symmetry parameter as a function of temperature; solid line was calculated from a Debye model with $\theta_{x}=300^{\circ} \mathrm{K}$ and $\theta_{z}=140^{\circ} \mathrm{K}$. 
corresponding mean square vibrational amplitudes are:

$$
\begin{aligned}
& \left\langle z^{2}\right\rangle=3.9 \times 10^{-18} \mathrm{~cm}^{2} \quad \text { and, } \\
& \left\langle x^{2}\right\rangle=0.9 \times 10^{-18} \mathrm{~cm}^{2} .
\end{aligned}
$$

This large difference in the mean square vibrational amplitudes is consistent with the layer-like crystal structure found for $\operatorname{CoCl}_{2}$.

C. CoF 2 Spectra Above the Néel Temperature

The observed variation in the relative intensity of $\mathrm{Fe}^{3+}$ and $\mathrm{Fe}^{2+}$ in the $\mathrm{CoF}_{2}$ spectra for. $\mathrm{T}>\mathrm{T}_{\mathrm{N}}$, as a function of temperature, may be due both to a difference in binding for the two ions and a variation with temperature in the relative number of the two ions. If the number of:iron ions in each charge state is assumed to be constant, the temperature variation in the relative intensity of the two lines, as measured by the area under the $\mathrm{Fe}^{2+}$ and $\mathrm{Fe}^{3+}$ spectra, can be used to calculate the ratio of the recoil-free fractions of the two ions as a function of temperature. For sources with negligible resonant self-absorption the area under the absorption curve is proportional to the recoil-free fraction of the source. ${ }^{29}$ Then for an absorber of constant thickness at. constant temperature the area under the absorption curve, as a function of source temperature, can be written

$$
S(T)=C f(T),
$$

where $f(T)$ is the recoil-free fraction of the source and $C$ is a proportionality. constant. The constant can be eliminated from consideration 
by normalizing the areas relative to some fixed temperature $\mathrm{T}_{0}$.

$$
\frac{S(T)}{S\left(T_{0}\right)}=\frac{f(T)}{f\left(T_{0}\right)}
$$

In this case $50^{\circ} \mathrm{K}$ was chosen as the normalization point instead of a very low temperature, because in the paramagnetic region errors in area measurement due to baseline uncertainty are minimized. The recoil-free fraction was calculated again using a Debye model in which the Debye temperature is replaced by a Mỏssbauer characteristic temperature.

Since the peaks near $v=0$ are not weli resolved, the areas under these peaks cannot be determined as precisely as those under the better resolved peaks at the more negative velocities, because of the strong correlation between the parameters. For this reason the analysis was carried out using only the areas under the two negative most peaks. This was justified by the fact that there was no observable Goldanski-Karyagin effect in the Mössbauer spectra, and thus, both peaks of a given quadrupole doublet have nearly the same area. The results are shown in Fig. 21 for both the $\mathrm{Fe}^{3+}$ and $\mathrm{Fe}^{2+}$ separately, and for the combined total area under both peaks. The curve drawn for $\theta_{\mathrm{m}}=330 \pm 10^{\circ} \mathrm{K}$ is a very good visual fit to the experimental data in this latter case. The solid curves drawn in the case of the individual peak areas represent the best visual fit at both extremes of the temperature range: The two values of $(500 \pm 50)^{\circ} \mathrm{K}$ for the $\mathrm{Fe}^{2+}$ data and $(280 \pm 30)^{\circ} \mathrm{K}$ for the $\mathrm{Fe}^{3+}$ data obtained for the characteristic Mo̊ssbauer temperatures indicate a large difference in binding for the two ions.

In order to determine whether this difference in binding, and hence 


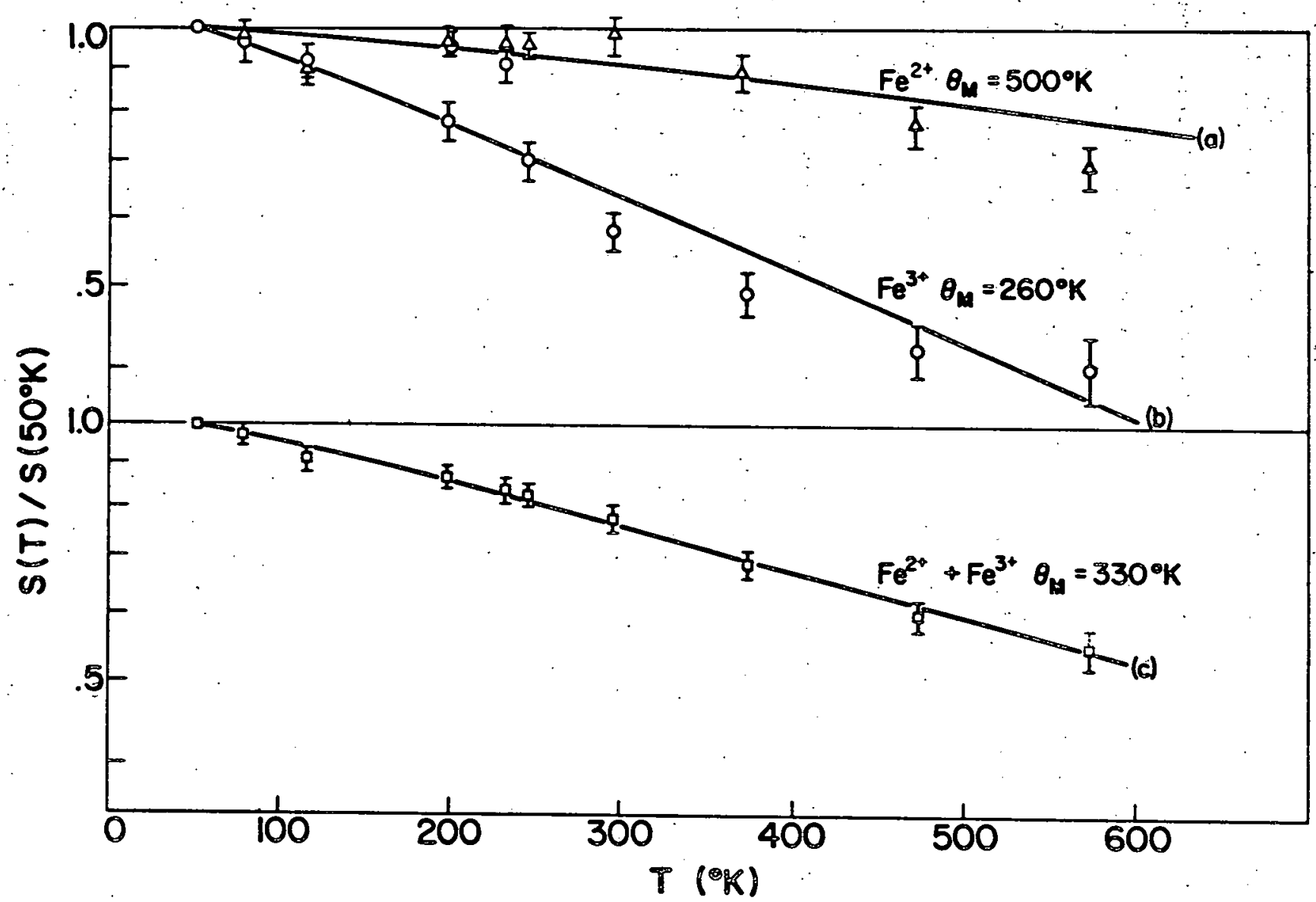

Figure 21. Temperature dependence of the relative area under the spectral peaks in CoF as a function of temperature. Solid lines are the results of calculations using a Debye model. a) area under the $\mathrm{Fe}^{2+}$ peak, $\theta_{\mathrm{m}}=500^{\circ} \mathrm{K}$; b) area under the $\mathrm{Fe}^{3+}$ peak, $\theta_{\mathrm{m}}=280^{\circ} \mathrm{K}$; c) total area under the entire curve $\theta_{\mathrm{m}}=330^{\circ} \mathrm{K}$. 
in recoil-free fractions, could explain the variation in intensity of the $\mathrm{Fe}^{2+}$ and $\mathrm{Fe}^{3+}$ lines as a function of temperature, the experimental area ratio $\mathrm{S}\left(\mathrm{Fe}^{3+}\right) / \mathrm{S}\left(\mathrm{Fe}^{2+}\right)$. was plotted and compared with the curve ca1culated by forming the ratio $\mathrm{f}\left(\mathrm{Fe}^{3+}\right) / \mathrm{f}\left(\mathrm{Fe}^{2+}\right)$ obtained from the previously mentioned recoil-free fractions for the Mössbauer characteristic temperatures. As shown in Fig. 22 this calculated curve follows the trend of the data, but the large error at the higher temperatures prevents a definitive conclusion as to possible changes in the relative number of ions in each of the two environments over the experimental temperature range.

The first moment of the spectra, however, is another index of changes in the ratio of the number of $\mathrm{Fe}^{2+}$ to $\mathrm{Fe}^{3+}$ ions. If this ratio is fixed over the experimental temperature range then the first moment of the Móssbauer spectra should increase monotonically with temperature, as the temperature dependent effect is then primarily the Josephson shift. ${ }^{31}$ The first moment was calculated by the formula

$$
\bar{v}=\Sigma v_{o i} s_{i} / \Sigma S_{i}
$$

where $\mathrm{V}_{o i}$, and $\mathrm{S}_{i}$ are the position and area of the $i^{\text {th }}$ spectral line, determined from a computer fit to the data.

The first moment, under this assumption, should increase monetonically with temperature as a result of the Josephson shift. In Fig. 23 the data points were calculated using the experimental areas and the solid curve illustrates the magnitude of the effect. From Figs. 22 and 23 we may conclude that, within experimental errors, the temperature 


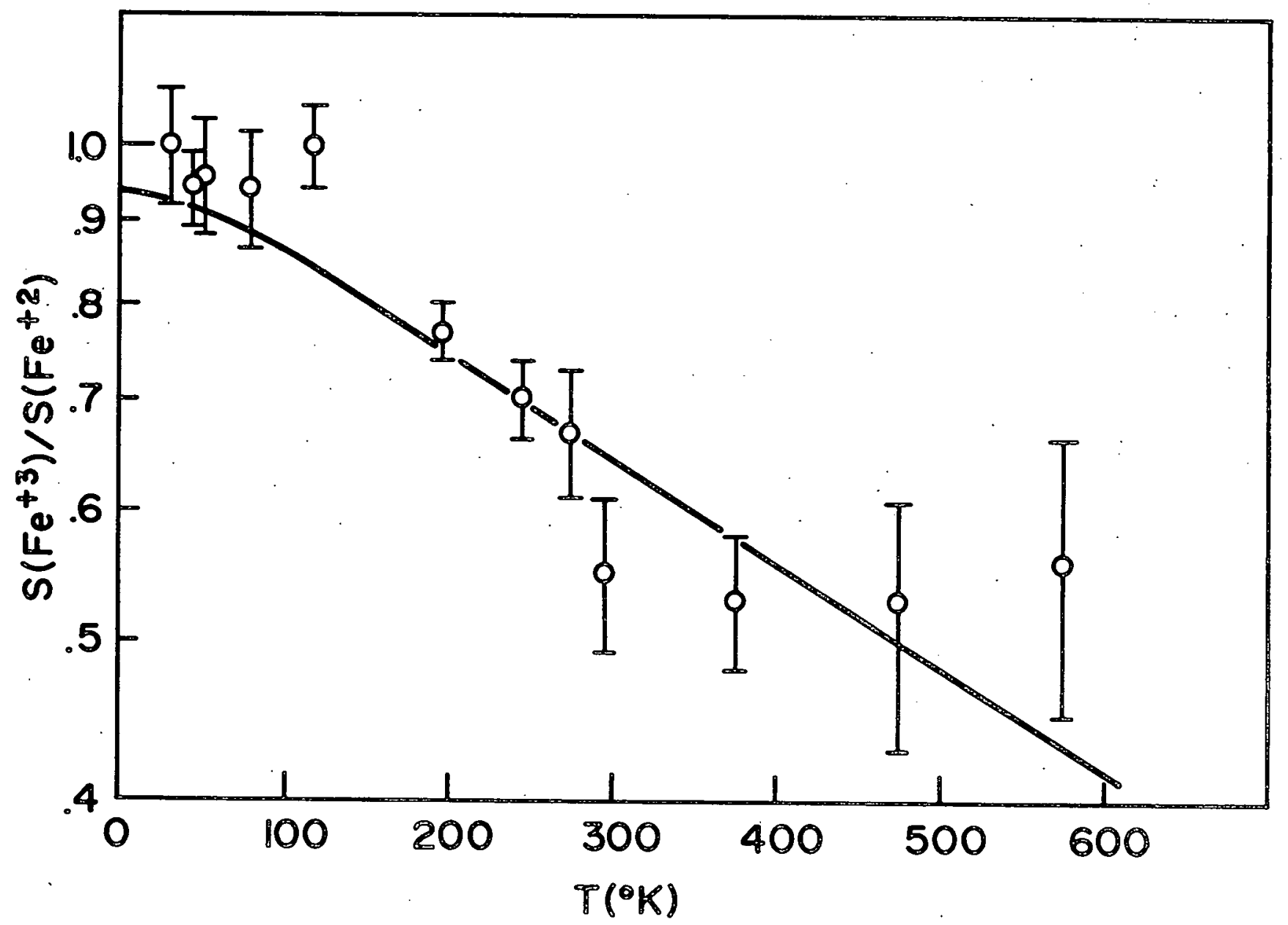

Figure 22. Ratio of the area under the $\mathrm{Fe}^{3+}$ and $\mathrm{Fe}^{2+}$ peaks as a function of temperature. The solid line is the ratio of recoil-free fractions calculated from a Debye model. 


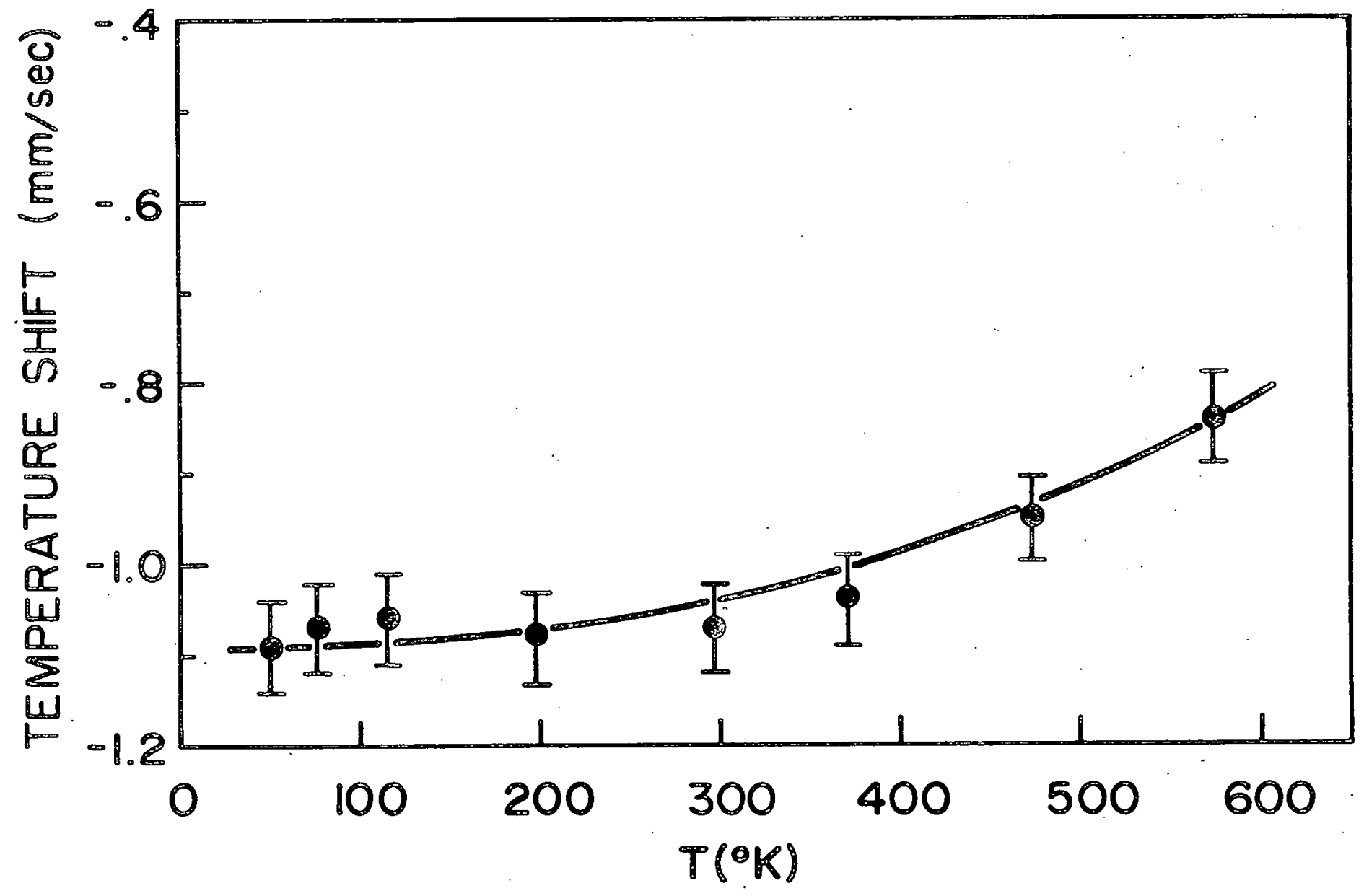

Figure 23. First moment of the $\mathrm{CoF}_{2}: \mathrm{Co}^{57}$ Mössbauer spectra as a function of temperature. 
variation of the recoil-free fraction alone can explain the observed variation in relative intensity of the $\mathrm{Fe}^{2+}$ and $\mathrm{Fe}^{3+}$ doublets in the $\mathrm{CoF}_{2}$ Mössbauer, spectra, although the errors are sufficiently great that the possibility of some conversion between the two types of sites should not be ruled out.

Since $\mathrm{CoF}_{2}$ and $\mathrm{FeF}_{2}$ have the same structure, one would expect an. iron ion to exist in $\mathrm{CoF}_{2}$ as a substitutional impurity and thus we would expect to observe only $\mathrm{Fe}^{2+}$ lines in the Mossbauer spectra. The origin of the $\mathrm{Fe}^{3+}$ lines observed in the actual spectra for $\mathrm{CoF}_{2}: \mathrm{Co}^{57}$ is one of the more interesting aspects of these experiments. The results of the measurements help to clarify the origin of these higher charge states. The failure to see the required broadening of the $\mathrm{Fe}^{3+}$. lines eliminates the type of Auger aftereffect, proposed by Wertheim ${ }^{9}$ for CoO, as the mechanism by which these two charge states are formed. Wertheim's model assumes that many of the $14.4 \mathrm{keV}$ gamma rays are emitted while the ion. is still in the $3+$. charge state which resulted from Auger emission following the K-capture decay of $\mathrm{Co}^{57}$. This mechanism requires that the time to reach electronic equilibrium from these higher charge states is of the same order of magnitude as the half life of the $14.4 \mathrm{keV}$ excited state, 100 nsec. In this case, however, the relevant time governing the linewidth of the gamma rays is the electronic equilibrium time. Thus, under this type of model, the gamma rays, emitted while the ion is in the. $\mathrm{Fe}^{3+}$ state, will have a shorter mean life, $\tau$, than those gamma rays emitted while the ion is in the $\mathrm{Fe}^{2+}$ state, when electronic equilibrium has been achieved: Hence, the width of the $\mathrm{Fe}^{3+}$. spectral lines, as given by the uncertainty relationship, $\Gamma=\hbar / \tau$, should be greater than 
the width of the $\mathrm{Fe}^{2+}$ 1ines. Since the measured linewidths of the $\mathrm{Fe}^{2+}$ and $\mathrm{Fe}^{3+}$ lines are equal within an experimental error of $5 \%$, it must be concluded.that the lifetime of the $\mathrm{Fe}^{3+}$ charge state in $\mathrm{CoF}_{2}$ is much longer than the $100 \mathrm{nsec}$ half life of the $14.4 \mathrm{keV}$ level.

Other possible causes of two charge states in the $\mathrm{CoF}_{2}$ Mössbauer spectra are non-stochiometry or a large number of lattice defects such as vacancies. The combined source-absorber experiments, however, tend to preclude these possibilities. Defects of this type might cause some of the $\mathrm{Co}^{57}$ atoms to have the $3+$ charge state in $\mathrm{CoF}_{2}$. This trivalent state could then be retained by the $\mathrm{Fe}^{57}$ ion after the radioactive decay process. The same defects, on the other hand, would cause $\mathrm{Fe}^{57}$ atoms doped into the sample, to also exist in $3+$ and $2+$ charge states, especially since iron tends to oxidize more readily than cobalt to the $3+$ state. The Mössbauer spectrum of $\mathrm{CoF}_{2}$ used as an absorber doped with $\mathrm{Fe}^{57}$, however, showed only the expected 2+ lines. This implies that the $\mathrm{Co}^{57}$ ions:are initially only in the $2+$ charge state when the sample is prepared by the method outlined in Chapter III.

The lower Mössbauer characteristic temperature associated with the $\mathrm{Fe}^{3+}$ lines indicates that these ions are less tightly bound and thus have a lower recoil-free fraction than the $\mathrm{Fe}^{2+}$ ions. If anion vacancies acting asielectron traps were responsible for the $\mathrm{Fe}^{3+}$ state, then one might expect a higher recoil-free fraction for these ions than for the $\mathrm{Fe}^{2+}$. ions because of the strong attraction of the highly electronegative fluorine ions for the higher charge state $\mathrm{Fe}^{3+}$ ion. The small ion size of $\mathrm{Fe}^{3+}$ will have to be taken into account, however, as it is opposite to the Coulomb effect. 
The fact that these extra $3+$ spectral lines are found only when the specimen doped with both $\mathrm{Co}^{57}$ and $\mathrm{Fe}^{57}$ is used as a source strongly suggests that the origin of these lines is associated with the $\mathrm{K}$-capture decay of $\mathrm{Co}^{57}$ and the subsequent Auger emission processes. In the case of $\mathrm{Fe}^{57}$ where Auger emission is the most probable mode of energy release as the holes in the $\mathrm{K}$ and subsequent shells are filled in the cascade following electron capture, higher charge states $\geq 3+$ will be created, albiet, for a time much shorter than the nuclear meanlife. The Coulomb forces associated with these higher charge states will produce very high local stresses in the lattice. These stresses, in turn, may produce a local distortion in the crystal structure in which the $\mathrm{Fe}^{3+}$ ion is stable. The $3 \mathrm{eV}$ recoil energy accompanying neutrino emission is clear1y inadequate to account for the proposed lattice distortion, because of the high characteristic temperature of this material.

This proposed mechanism by which multiple charge states may be produced in the Mössbauer spectra of cobalt compounds is supported by the work of other investigators. Wertheim ${ }^{47}$ has measured the Mössbauer effect in cobaltic acetylacetonate $\left[\mathrm{Co}(\mathrm{III})(\mathrm{acac})_{3}\right]$ and ferric acetylacetonate $\left[\mathrm{Fe}(\mathrm{III})(\mathrm{acac})_{3}\right]$. With the former compound used as a source containing $\mathrm{Co}^{57}$, resonant absorption lines with the characteristic isomer shifts of both $\mathrm{Fe}^{3+}$ and $\mathrm{Fe}^{2+}$ were observed. On the other hand, the latter compound used as an absorber showed only a single spectral line with the expected $\mathrm{Fe}^{3+}$ isomer shift. In this case it was also proposed that the local distortion, produced in accomodating the initial high charge states, formed an environment in which the $\mathrm{Fe}^{2+}$ ion was stable in certain cases. A local lattice distortion as the origin of the multiple charges 
in Mösbauer source spectra was first proposed by Mullen and $\mathrm{Ok}^{11}$ after their systematic study of the hydrated cobalt chloride compounds. In this case the nuclear recoil energy of the order of $3 \mathrm{eV}$ arising from the neutrino emission accompanying $\mathrm{K}$-capture was postulated as the mechanism: by which the local distortion was formed, although distortions due to transient Auger aftereffects $\left(\ll 10^{-7} \mathrm{sec}\right)$ would be compatible with their observations on these compounds.

In the two cases mentioned above the binding energy of the cobalt ion is relatively weak as indicated by the size of the Mössbauer effect in these compounds. In contrast, the multiple charge states in the $\mathrm{CoF}_{2}: \mathrm{Co}^{57}$ source spectra are the first such observations on a substance in which the binding is quite large, as indicated by the $500^{\circ} \mathrm{K}$ characteristic Mössbauer temperature found for the $\mathrm{Fe}^{2+}$. spectra in $\mathrm{CoF}_{2}$.

An interesting experiment in regard to this question of multiple charge states has been performed by. Jung and Triftshauser ${ }^{48}$ using the Móssbauer effect in $\mathrm{Te}^{125}$. This isotope is the daughter nucleus of both the $\beta^{-}$decay of $\mathrm{Sb}^{125}$ and the K-capture decay of $\mathrm{I}^{125}$. The Mössbauer spectra of a source such as $\mathrm{Na} \mathrm{Sb}{ }^{125} 0_{3}$ showed only a single line while the source spectra of $\mathrm{Na} \mathrm{I}^{125} \mathrm{O}_{3}$ showed multiple charge state lines. This suggests again that $\mathrm{K}$-capture and the subsequent processes, including Auger emission, can modify the chemical binding of the daughter atom in a solid.

\section{Hyperfine Field Parameters}

Karyagin's ${ }^{6}$ "combinatorial method" for determining the hyperfine field parameters, in the general case of a combined magnetic dipole and 
electric quadrupole. interaction, requires that all eight line positions be known in order to determine the eigenvalues of the excited state. In the cases considered here it was not possible to resolve all eight lines. For $\mathrm{CoCl}_{2}$ only four peaks. were resolvable, but the position of six of the lines were found by fitting the data to a superposition of Lorentzian curves. Similar difficulties were encountered in the analysis of the $\mathrm{CoF}_{2}$ data, in addition to the problem of the simultaneous presence of both the $\mathrm{Fe}^{2+}$ and $\mathrm{Fe}^{3+}$ hyperfine patterns. After considerable effort curve fitting procedures yielded the position of six peaks in each of the two spectra, when the restrictive assumptions discussed in Chapter IV were used: As the final analysis indicated, the lack of resolution of the two additional lines in the $\mathrm{CoF}_{2}-\mathrm{Fe}^{2+}$ spectrum was due to the very small intensity of one of the lines and the coalescing of two lines into a single peak.

The determination of the position of only six lines in the hyperfine pattern requires a modification of Karyagin's method for determining the eigenvalues of these line positions. There will be relatively intense transitions from the two excited state levels, which have large components of the $|3 / 2,1 / 2\rangle$ land $|3 / 2,-1 / 2\rangle$ wavefunctions in their eigenstates, to each of the two ground state levels. The four lines resulting from these transitions can be identified on a trial and error basis. If a $4 \times 4$ array is constructed, whose elements are the permutations of all differences in line position of four of the spectral lines, then six positive non-zero numbers will appear in the array. If the four lines chosen to construct the array are those arising from the transitions mentioned above, then the difference in energy between the excited 
state levels will appear twice in the group of six numbers. The difference between ground state levels will likewise appear:twice in this same group. The remaining two numbers are the sum and difference of these two energy differences, and they give a consistency check on the proper choice of the four lines with which the array is constructed.

Let the positions of the four lines, which represent the shift from the center of the spectrum, be denoted as $\mathrm{L}_{2}, \mathrm{~L}_{3}, \mathrm{~L}_{4}, \mathrm{~L}_{5}$. This choice of subscript is made since the four lines which form the combinatorial table will be the inner four lines of the six line pattern in the majority of cases. Now, if the difference between $L_{i}$ and $L_{j}$ is the ground state splitting $\Delta \mathrm{E}_{\mathrm{G}}$, then these lines originate from the same excited state level $\mathrm{E}_{k}$, and

$$
\begin{aligned}
& L_{i}=E_{k}+\Delta E_{G} / 2, \\
& L_{j}=E_{k}-\Delta E_{G} / 2,
\end{aligned}
$$

and thus,

$$
E_{k}=\frac{1}{2}\left[L_{i}+L_{j}\right]
$$

The eigenenergies which are associated with the remaining two lines $\mathrm{L}_{1}$ and $\mathrm{L}_{6}$ can be found either through the relationship

$$
\mathrm{E}_{\mathrm{n}}=\mathrm{L}_{1}-\Delta_{\mathrm{G}} / 2
$$

or

$$
E_{n}=E_{k}+\left(L_{1}-L_{k}\right)
$$

where $L_{k}$ is the line associated with the transition from state $E_{k}$ to 
the same sublevel of the ground state as $\mathrm{L}_{1}$. A similar method is used to find $\mathrm{L}_{6}$. The resultant eigenenergies must now be corrected for the isomer shift of the spectrum, since Karyagin's method of analysis requires that $\Sigma_{n} E_{n}=0$.

Unlike the full 8-line combinatorial method of Karyagin, the procedure outlined above has an ambiguity with regard to distinguishing between the ground state splitting and the excited state splitting. Consequently, two sets of eigenvalues result from the analysis. This ambiguity can be resolved, however, by using the derived parameters to calculate the spectrum and comparing the calculated intensities of the lines with the experimentally observed values. In practice, the improper choice of the ground state splitting can sometimes be recognized by the negative values for the absolute value of the matrix elements of the excited state Hamiltonian, which occur in the analysis.

This method of analysis was applied to magnetic hyperfine patterns of both $\mathrm{CoCl}_{2}$ and $\dot{\mathrm{CoF}}_{2}$. The estimates of the local field parameters thus obtained were then used.as the initial starting point for a least squares fitting to the experimental spectra, using the approach of ok and Mullen ${ }^{7}$ in combination with the nonlinear regression curve fitting program. This latter program was modified in such a way that the number of times the excited state Hamiltonian, matrix had to be diagonalized for each iteration was reduced to seven. With these programing features a fit was obtained in less than one minute of computer time.

In the case of $\mathrm{Fe}$ in $\mathrm{CoCl}_{2}$, the best fit to the data at $10^{\circ} \mathrm{K}$ was found for the following values of the parameters which describe the local field at the nucleus: 


$$
\begin{aligned}
\mathrm{H} & =+49 \pm 2 \mathrm{k} 0 \mathrm{e} \\
\frac{1}{2} \mathrm{e}^{2} \mathrm{qQ} & =+1.16 \pm .06 \mathrm{~mm} / \mathrm{sec} \\
\theta & =90^{\circ} \\
\phi & =195^{\circ} \\
& \cdot \\
\eta & =0.34 \pm 0.1
\end{aligned}
$$

The eigenenergies of the four sublevels of the excited state are in units of the Doppler shift velocity $(\mathrm{mm} / \mathrm{sec})$ :

$$
\begin{aligned}
& \mathrm{E}_{1}=0.84, \\
& \mathrm{E}_{2}=0.50 ; \\
& \mathrm{E}_{3}=-0.36, \\
& \mathrm{E}_{4}=-0.98 .
\end{aligned}
$$

The relatively small value of $+49 \mathrm{k} 0 \mathrm{e}$ indicated by the present experiment for the magnetic field at an $\mathrm{Fe}^{57}$ nucleus in $\mathrm{CoCl}_{2}$ at $10^{\circ} \mathrm{K}$ differs from that measured for most ferrous ions in antiferromagnetic compounds. This value is consistent, however, with the magnitude of the internal magnetic field found in other metamagnetic compounds. Ono, Ito and Fujita ${ }^{19}$ observed zero magnetic field at the iron nucleus in $\mathrm{FeCl}_{2}$ for temperatures as low as the $\lambda$-point of helium, and they estimate the internal field to be $78 \mathrm{k} 0 \mathrm{e}$ in the case of $\mathrm{FeI}_{2}{ }^{49}$ at $4.2^{\circ} \mathrm{K}$. Recently, Simkin ${ }^{20}$. has confirmed the zero field measurements in $\mathrm{FeCl}_{2}$ and has measured a $30 \mathrm{kOe}$ internal field in $\mathrm{FeBr}_{2}$ below its Néel temperature. The null result in $\mathrm{FeCl}_{2}$ was attributed to a cancellation of the orbital, Fermi contact and dipole contributions to the magnetic field at the nucleus. The small positive values observed for the internal field at the iron nucleus in $\mathrm{CoCl}_{2}, \mathrm{FeI}_{2}$ and $\mathrm{FeBr}_{2}$ indicate a tendancy 
towards similar cancellations of the hyperfine field in the metamagnetic layer compounds.

The analysis of the $\mathrm{CoF}_{2}$ data yielded the following parameters for the local field at the $\mathrm{Fe}^{2+}$ nucleus at $11^{\circ} \mathrm{K}$ :

$$
\begin{aligned}
\mathrm{H} & =-245 \pm 2 \mathrm{k0e} \\
\frac{1}{2} \mathrm{e}^{2} \mathrm{qQ} & =+3.02 \pm .06 \mathrm{~mm} / \mathrm{sec} \\
\theta & =84 \pm 5^{\circ} \\
\phi & =4 \pm 6^{\circ} \\
\eta & =0.31 \pm .05
\end{aligned}
$$

The first excited state of the $\mathrm{Fe}^{57}$ nucleus splits under the influence of this field into four states with eigenenergies:

$$
\begin{aligned}
& E_{1}=+2.73, \\
& E_{2}=+1.72, \\
& E_{3}=-1.00, \\
& E_{4}=-3.45,
\end{aligned}
$$

where the convenient Mössbauer units of $\mathrm{mm} / \mathrm{sec}$ are again used.

The magnitude of field at the nucleus of the $\mathrm{Fe}^{3+}$ ion is given by the expression, $\mathrm{H}=\Delta \mathrm{V} /\left|3 \mathrm{~g}_{1}-\mathrm{g}_{0}\right|$, with the assumption that the quadrupole interaction is negligible. The measured overall splitting, $\Delta V$, of the $\mathrm{Fe}^{3+}$ pattern at $11^{\circ} \mathrm{K}$ was $17.46 \pm .05 \mathrm{~mm} / \mathrm{sec}$. This corresponds to a magnetic field at the nucleus of $-545 \pm 5 \mathrm{k} 0 \mathrm{e}$. The sign here is assumed negative in keeping with the established results for other similar $\mathrm{Fe}^{3+}$ materials.

The Mỏssbauer spectra calculated from the above hyperfine field 
parameters and the experimental linewidths are shown in Fig. 24 along with the experimental data. The position and relative intensities of the eight spectral lines are indicated schematically. In the case of $\mathrm{CoF}_{2}$, the $\mathrm{Fe}^{3+}$ pattern has been numerically subtracted from the data.

The sign of the internal magnetic field in the $\operatorname{CoCl}_{2}$ case was determined from the fact that the electric field gradient component was previously determined to be positive. The relative intensities and line positions of the spectra, as calculated by diagonalizing the excited state Hamiltonian, are very sensitive to the relative signs of $\mathrm{H}$ and q. If the sign of one is known, the other can be determined unambiguous $1 \mathrm{y}$.

If one then assumes that the sign of $\mathrm{H}$ is negative in the case of $\mathrm{Fe}$ in $\mathrm{CoF}_{2}$, as it is with most $\mathrm{Fe}^{2+}$ magnetic hyperfine spectra, including the similar material $\mathrm{FeF}_{2}$, then the sign of $\mathrm{q}$ is positive in this case.

The use of the Mosssbauer effect with $\mathrm{Fe}^{57}$ to measure the Neel temperature of compounds in which the $F e$ ion is an impurity has been open to question ${ }^{50}$. Since the $\mathrm{Fe}^{57}$ ion is a substitutional impurity in such experiments, it might be argued that the Néel temperature measured with the Mössbauer effect in an impurity nucleus would be different than that of the host. Iattice, because of differences in host-host and hostimpurity exchange interactions. There is evidence, however, that the Mössbauer determination of the Néel temperature with impurity atoms do, in fact, give the Nél temperature of the host lattice.. Measurements of the Néel temperature using $\mathrm{Fe}^{57}$ in $\mathrm{CoO}^{7}$ and $\mathrm{MnF}_{2}^{50}$ yielded the same value as that obtained from macroscopic measurements. The present work 

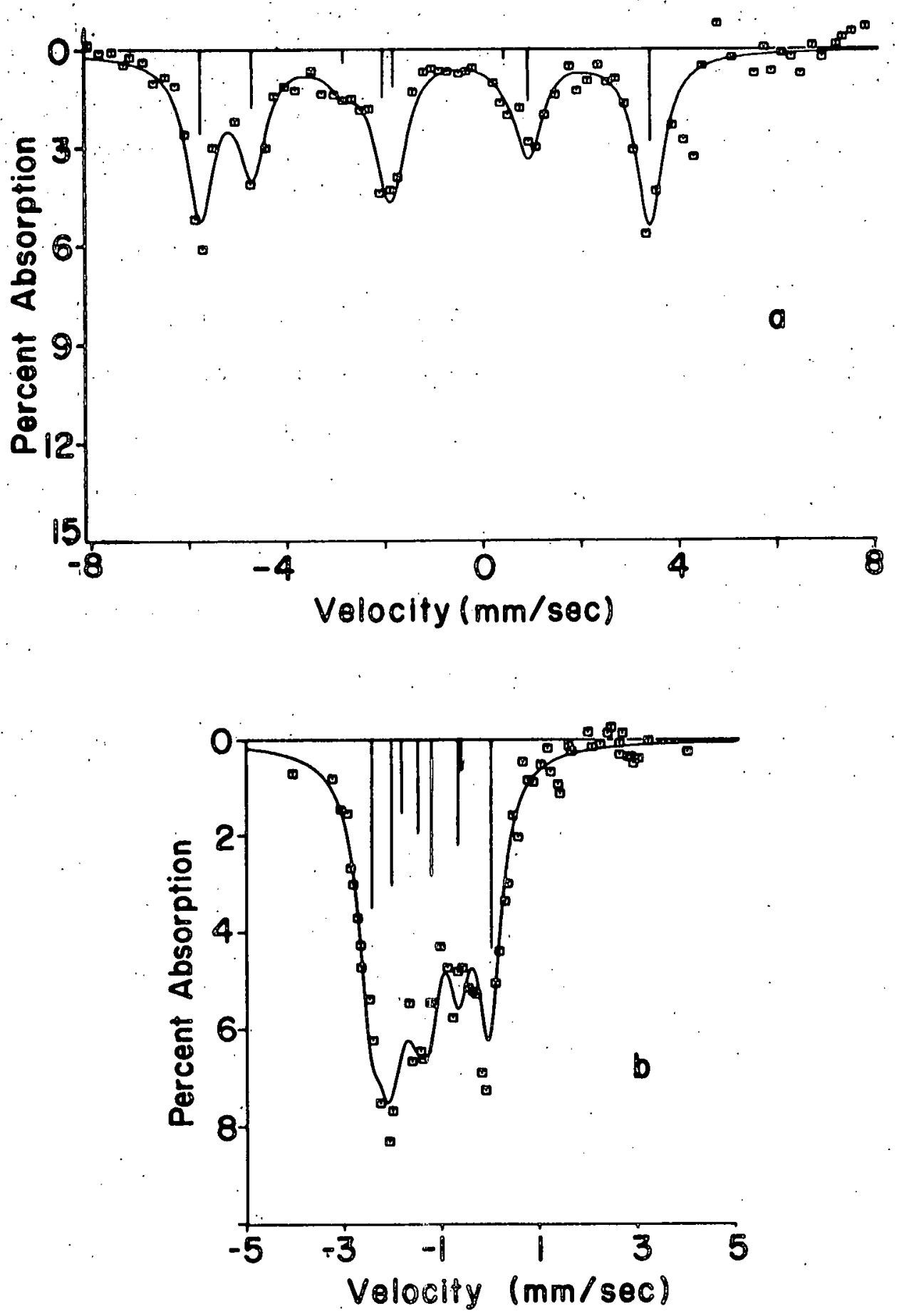

Figure 24. Experimental Mössbauer hyperfine spectra and the resonant emission spectra calculated from estimates of the hyperfine field parameters. The position and intensities of the spectral lines are indicated schematica11y. a) $\operatorname{CoF}_{2}, T=11^{\circ} \mathrm{K}$, b) $\operatorname{CoC}_{2}, T=10^{\circ} \mathrm{K}$. 
gave similar results in that the Néel temperature for both $\mathrm{CoCl}_{2}$ and $\mathrm{CoF}_{2}$ as measured with the Mössbauer effect are in agreement with macroscopically determined values.

Erickson's ${ }^{25}$ estimates of the Néel temperature for the transition element fluorides are consistent 1 y $10-15^{\circ} \mathrm{K}$ higher than Stout and Catalano's ${ }^{26}$ specific heat values. Martel, Cowley, and Stevenson ${ }^{51}$ have repeated Erickson's measurements for $\mathrm{CoF}_{2}$ very recently. They find a Néel temperature of $37.7 \pm .5^{\circ} \mathrm{K}$ which resolves the apparent discrepancy in the earlier work, and also agrees with the present experimental results.

The fact that both the $3+$ and $2+$ magnetic hyperfine patterns appeared in the Mössbauer spectra of $\mathrm{CoF}_{2}: \mathrm{Co}^{57}$ is a further indication that the Neel temperature as measured with the Mossbauer effect in impurity atoms is identical to that of the host lattice. 


\section{SUMMARY}

The Móssbauer effect in $\mathrm{Fe}^{57}$ has been used to study the internal fields in both $\mathrm{CoCl}_{2}$ and $\mathrm{CoF}_{2}$. The spectra have been taken both above and below the Néel temperatures of the two compounds.

In the temperature range from just above the Néel temperature of $25^{\circ} \mathrm{K}$ to $670^{\circ} \mathrm{K}$, the Moss bauer spectrum of $\mathrm{CoCl}_{2}$ is a doublet. Both the magnitude and temperature dependence of the doublet splitting are in quantitative agreement with the calculations, based on an application of crystalline field theory to calculate the electric field gradient in ferrous ions. A fit to the data gives the values of $\Delta=190 \pm 25 \mathrm{~cm}^{-1}$ for the trigonal field splitting and $\alpha^{2}=0.7 \pm 0.1$ for the covalency factor.

These spectra also show an asymmetry which increases monatonically with temperature. This asymmetry has been interpreted as an example of the Goldanski-Karyagin effect which arises from an anisotropic crystal binding of the $\mathrm{Fe}^{57}$ ion in $\mathrm{CoCl}_{2}$. Analysis of the data enables us to describe the lattice vibrations parallel and perpendicular to the c-axis in terms of two characteristic Mo̊ssbauer temperatures, $\theta_{z}=140^{\circ} \mathrm{K}$ and $\theta_{\mathrm{x}}=300^{\circ} \mathrm{K}$.

The Mo̊ssbauer spectra of $\mathrm{CoF}_{2}: \mathrm{Co}^{57}$ above its indicated Néel temperature of $38 \pm 1^{\circ} \mathrm{K}$ consists of two superimposed doublets. One doublet has the splitting and isomer shift charactertistic of a ferrous ion, 
while the other has the characteristics of a ferric ion. These multiple charge states appear only in the source spectra. A sample doped with both $\mathrm{Co}^{57}$ and $\mathrm{Fe}^{57}$ showed both doublets in its spectra when used as a source, while only the ferrous, $\mathrm{Fe}^{2+}$, doublet was observed in the Mössbauer spectrum of this sample when it was used as an absorber.

An analysis of the temperature dependence of the intensity of the doublets, as measured by the area under the absorption curves, which is proportional to the recoil-free fraction, shows that the $\mathrm{Fe}^{2+}$ ion, formed by the decay of $\mathrm{Co}^{57}$, is more tightly bound in $\mathrm{CoF}_{2}$ with a characteristic temperature of $\theta_{\mathrm{m}}=500 \pm 50^{\circ} \mathrm{K}$ than the $\mathrm{Fe}^{3+}$ ion whose measured characteristic temperature is only $280 \pm 30^{\circ} \mathrm{K}$.

Although multiple charge states have been observed in Mössbauer spectra of non-metallic sources doped with $\mathrm{Co}^{57}$, this is the first observation of such effects in a source with such a high characteristic Mo̊ssbauer temperature, $\theta_{\mathrm{m}}$.

The experiments performed with $\mathrm{CoF}_{2}$ eliminate both non-stochiometry and the Auger aftereffect, of the type proposed by Wertheim for the case of $\mathrm{COO}$, as the possible origin of the $\mathrm{Fe}^{3+}$ charge state in these spectra. In light of this work and that of others, it is proposed that a local lattice distortion caused by the Coulomb forces, arising from the high ionic charge states which are produced by $\mathrm{K}$-capture and subsequent transient electronic processes, results in a local environment in which the $\mathrm{Fe}^{3+}$ ion is stable for a time which is much greater than the mean life of the first excited state of $\mathrm{Fe}^{57}$.

Below the Néel temperatures the Mo̊ssbauer spectra of both compounds are complex patterns. Both can be interpreted, however, in terms of a 
combined magnetic dipole and electric quadrupole interaction acting upon the excited state of the nucleus. In $\mathrm{CoCl}_{2}$ the strength of the magnetic field is estimated to be $+49 \pm 2 \mathrm{k} 0 \mathrm{e}$ at $10^{\circ} \mathrm{K}$. The magnitude of the field at the nucleus of an $\mathrm{Fe}^{2+}$ ion in $\mathrm{CoF}_{2}$ at $11^{\circ} \mathrm{K}$ is found to be $-245 \pm 2 \mathrm{k} 0 \mathrm{e}$, and a value of $-545 \pm 5 \mathrm{k} 0 \mathrm{e}$ is found for the field at the nucleus of the $\mathrm{Fe}^{3+}$ ion: Estimates were also obtained for the values of the other parameters describing the internal field at the nucleus. The determination of the sign of $\mathrm{V}_{z z}$ component of the electric field gradient in $\mathrm{CoCl}_{2}$ from applied magnetic field measurements and crystalline field calculations made it possible to determine the sign of the internal magnetic field in this compound as positive. This result is in agreement with measurements made with other metamagnetic layer compounds.

The measured Neel temperatures for both $\mathrm{CoCl}_{2}$ and $\mathrm{CoF}_{2}$ of $25 \pm 2^{\circ} \mathrm{K}$ and $38 \pm 1^{\circ} \mathrm{K}$ respectively are in agreement with earlier measurements. This supports the current view ${ }^{7,50}$ that the Neel temperature measured by the Mössbauer effect with an impurity nucleus yields the same Neel temperature as that of the host lattice. 
LIST OF REFERENCES 
LIST OF REFERENCES

1. R. L. Mössbauer, Z. Physik, 151; 129 (1958).

2. W. E. Lamb, Phys. Rev. 55, 136 (1938).

3. G. K. Wertheim, Nucleonics, 19, 52 (1961).

4. W. Kundig, Nucl. Instr. and Methods, 48, 219 (1967).

5. G. R. Hoy and S. Chandra, J. Chem. Phys. 47, 961 (1967).

6. S. V. Karyagin; Soviet Physics-Solid State, 으, 391 (1968).

7. H. N. Ok and J. G. Mullen, Phys. Rev. 168, 550, 563, (1968).

8. Muir, Ando and Coogan, Mössbauer Effect Data Index 1958-1965, Interscience Publishers, New York, 1966.

9. G. K. Wertheim, Phys. Rev. 124, 764 (1961).

10. R. D. Evans, The Atomic Nucleus, McGraw-Hill, New York, 1955, p. 565.

11. J. G. Mullen and H. N. Ok, Phys. Rev. Letters, 17, 287, (1966).

12. W. Trifthauser and P. P. Craig, Phys. Rev. Letters, 16, 1161 (1966).

13. R. Inga 11 s and G. DePasquali, Phys. Letters, $\underline{15}, 262$ (1965).

14. C. Starr, F. Bitter, and A. R. Kaufmann, Phys. Rev. 58, 977 (1940).

15. Bizette, Terrier, and Tsai, Compt. Rendu, 245, 507 (1957).

16. R. G. W. Wychoff, Crystal Structures, Interscience Publishing Co. Inc., New York, 1960, Vol. I, Table IV.7.

17. L. Landau, Physik. Z. Sowjet Union, 4, 675 (1933).

18. M. K. Wilkinson, T. W. Cable, E. O. Wollan, and W. C. Koether, Phys. Rev. 113, 497 (1959).

19. K. Ono, A. Ito, and T. Fujita, J. Phys. Soc. Japan, 19, 2119 (1964). 
20. D. J. Simkin, Phys. Rev. (to be published) 1969.

21. V. I. Goldanski, et. al., Proc. Acad. Sci. USSR, Phys. Chem. Sect. 147,766 (1963).

22. S. V. Karyagin, Proc. Acad. Sci. USSR,.. Phys. Chem. Sect. 148, 110 (1964).

23. R. G. W. Wychoff, Crystal Structures, Interscience Publishing Co. Inc., New York, 1960, Vol. I; Table IV.3..

24. H. Bizette, Ann. Physick, 12, 295: (1946).

25. R. A. Erickson, Phys. Rev. 90; 779 (19.53).

26. J. W. Stout and E. Catalano, Phys. Rev. 92,, 2013,.(1955).

27. H. J. Lipkin, Annals of Physics, 9., 332 (1960).

28. H. Fraunfelder, The Mössbauer:Effect, W. A. Benjamin, Inc: New York, 1963, p. 31.

29. S. Margulies and J. R. Ehrman, Nucl. Instr. and Methods, 12, 131 (1961):

30. 0. C. Kistner and A. W. Sunyar, Phys. Rev. Letters, 4, 412 (1960).

31. B. D. Josephson, Phys. Rev. Letters, 4, 342 (1960).

32. R. V. Pound and G. A. Rebka Jr., Phys. Rev. Letters, 4, 274 (1960).

33. M. H. Cohen and F. Reif, Solid State Physics, edited by F. Seitz and P: Turnbu11, Academic Press Inc., New York 1957, Vo1. 5.

34. Yu. Kagan, Soviet Physics-Doklady, 6, 881 (1962); V. I. Goldenski;. E. F. Makarov, I. P. Suzdelev, and $\bar{I}$. A. Vinogradov, Phys. Rev. Letters $\underline{20}, 137$ (1968).

35. R. M. Sternheimer and H. M. Foley, Phys. Rev. 102, 731 (1964).

36. R. Inga1ls, Phys. Rev 1 133, A787 (1964).

37. B. Bleaney and K. W. H. Stevens, Repts. Progr. Phys. 16, 108 (1953).

38. J. Kanamori, Progr. Theort. Physics (Kyoto) 17, 177 (1957).

39. Eugen Merzbacher, Quantum Mechanics, John Wiley \& Sons, Inc. New York (1962) p. 401.

40. J. Owen, Proc. Roy. Soc. (London), A227, 183 (1955). 
41. J. G. Mullen, Phys. Rev. 131, 1410 (1963).

42. R. C. Knauer and J. G. Mullen, Rev. Sci. Instr. 38, 1624 (1967).

43. Therma1 Conductivity of Metals and Alloys at Low Temperatures, National Bureau of Standards Circular 556, (1954).

44. J. D. G. Linsay, Rev. Sci. Instr. 37, 1'192 (1966).

45. B. W. Marquardt, J. Soc. Indust. and App1. Math. 11, 431 (1963).

46. X-ray Powder Data: File, ASTM Special Technical Publication 48-J, 1960, Index card 3-0409.

47. G. K. Wertheim et. al. J. Chem. Phys. 37, 687 (1962).

48. P. Jung and W. Triftshauser, Phys. Rev. 175, 512 (1968).

49. Fujita, Ito and Ono, J. Phys..Soc. Japan, 21, 1743 (1966).

50. G. K. Wertheim, H. J. Guggenheim, and D. N. E. Buchanan, Phys. Rev. Letters. 20, 1158 (1968).

51. P. Martel, R. A. Cowley, and R. W. H. Stevenson, Can. J. Phys. $\underline{46}, .1355$ (1968). 